\title{
WestVirginiaUniversity
}

THE RESEARCH REPOSITORY @ WVU

Graduate Theses, Dissertations, and Problem Reports

2019

\section{Analyzing Mathematicians' Concept Images of Differentials}

Timothy Shawn McCarty

tmccarty@mix.wvu.edu

Follow this and additional works at: https://researchrepository.wvu.edu/etd

Part of the Other Mathematics Commons

\section{Recommended Citation}

McCarty, Timothy Shawn, "Analyzing Mathematicians' Concept Images of Differentials" (2019). Graduate Theses, Dissertations, and Problem Reports. 4062.

https://researchrepository.wvu.edu/etd/4062

This Dissertation is protected by copyright and/or related rights. It has been brought to you by the The Research Repository @ WVU with permission from the rights-holder(s). You are free to use this Dissertation in any way that is permitted by the copyright and related rights legislation that applies to your use. For other uses you must obtain permission from the rights-holder(s) directly, unless additional rights are indicated by a Creative Commons license in the record and/ or on the work itself. This Dissertation has been accepted for inclusion in WVU Graduate Theses, Dissertations, and Problem Reports collection by an authorized administrator of The Research Repository @ WVU.

For more information, please contact researchrepository@mail.wvu.edu. 


\title{
ANALYZING MATHEMATICIANS' CONCEPT IMAGES \\ OF DIFFERENTIALS
}

\author{
Tim McCarty
}

\author{
Dissertation submitted \\ to the Eberly College of Arts and Sciences \\ at West Virginia University \\ in partial fulfillment of the requirements for the degree of \\ Doctor of Philosophy in \\ Mathematics
}
Vicki Sealey, Ph.D., Chair Jessica Deshler, Ph.D.
John Goldwasser, Ph.D.
Nicole Engelke Infante, Ph.D. John Stewart, Ph.D.

Department of Mathematics

\author{
Morgantown, West Virginia \\ 2019
}

Keywords: Differentials, Concept Image, Calculus, Derivative, Integral Copyright 2019 Tim McCarty 


\section{ABSTRACT \\ Analyzing Mathematicians' Concept Images of Differentials}

Tim McCarty

The differential is a symbol that is common in first- and second-year calculus. It is perhaps expected that a common mathematical symbol would be interpreted universally. However, recent literature that addresses student interpretations of differentials, usually in the context of definite integration, suggests that this is not the case, and that many interpretations are possible. Reviews of textbooks showed that there was not a lot of discussion about differentials, and what interpretations there were depended upon the context in which the differentials were presented. This dissertation explores some of these issues. Since students may not have the experience necessary to build their own interpretations totally free of their instructors' influences, I chose to interview experienced mathematicians for their differential interpretations. Most of the current literature involves the differential within the context of definite integrals; my work expands on this literature by exploring additional expressions that contain differentials. The goal was to build a dataset of multiple instructors' interpretations of multiple differentials to see how uniform those interpretations were.

Initial interviews discussing five expressions which contained differentials, three contexts in which most of these expressions were used, and auxiliary questions that asked the meaning of "differential," the differences between $d x$ and $\Delta x$, and the interpretation of phrases used to describe infinitely small quantities were conducted with seven expert mathematicians from a large research university. By analyzing the responses given by these mathematicians, two lists of themes were created: one based on remarks that address the quality of the differential directly, and one based on remarks that address one's feelings about differentials. In addition, for the responses that address differentials directly, a flowchart was created to guide each of these responses to its proper theme. After the creation of these lists, three more mathematicians were interviewed to ensure that the theme lists would still be valid outside of the interviews used to create them.

Not only was no overall formal concept image for the differential found, but many different and sometimes contrasting themes were found within each interview subject's personal concept image. A framework for categorizing the multiple conceptualizations that were found for the differentials themselves was created, as well as a beginning list of ancillary themes that address possible thoughts about and uses of differentials. The dissertation concludes with a list of possible teaching implications that might arise from the existence of multiple differential conceptualizations, as well as some suggested future research that might expand upon this work. 


\section{DEDICATION}

To my mother, Betty McCarty, whose love, support, and help has been a precious gift, not just during the creation of this work, but throughout my entire life.

This dissertation is as much a tribute to her as it is a requirement for me. 


\section{ACKNOWLEDGEMENTS}

Thanks to my family: Dad, Donna, Terry, Jereme, John, Cindy, Colsen, and a myriad of aunts, uncles, and cousins. Having your love and support throughout the years has helped me get this far - may this love and support continue beyond this dissertation!

A very special thanks to my advisor, Dr. Vicki Sealey, who was a constant source of positivity and encouragement through my ever-changing, sometimes-depressed-and-sometimeseuphoric moods, through numerous meetings and discussions, through countless permutations of the data set that were always "almost, but not quite correct", and through many, many gold stars. May I be as helpful to my future students.

Thanks to the other members of my dissertation committee: Dr. Jessica Deshler, for her support during my early semesters of research and her work with the GTA's, Dr. John Goldwasser for his helpful comments and for stepping in at the last minute, Dr. Nicole Engelke Infante, for allowing me into some of her research, teaching me a lot about everything, and always having answers to my many and varied questions, and Dr. John Stewart, for bringing to our committee discussions a "Physics" perspective and many thoughts I would never have otherwise considered.

A special thank you to the wonderful people at Scott's Run Settlement House: Deb, Ira, Shay, Courtney, Becca, Pete, and Jen. All I needed from you was somewhere to go get volunteer hours for my fellowship - but in addition to that, you gave me a safe haven full of love, support, and friendship and the opportunity to change myself and make me a better man by helping you in your charitable work. You all saved my life and I will never forget it. Additional thanks to my volunteer buddies Erina, Julie, Kristen, Maverick, P.J., Chuck, Vicki, Allyson, Allisynne, Isabella, and Juliana - you made this past year of volunteering so much better! 
My thanks to my WVU RUME cohort: the "integration gang” Krista, Cody, Abby, and Olga, Keith and Ming for being conference roommates and sounding boards, Jenny, Samantha, Jaylyn, Heather, and Morgan for being parts of great RUME classes and/or research groups, and special thanks to Josh, who was a good friend to me, and whose intelligence and enthusiasm and excitement for my work (which sometimes dwarfed my own!) inspired me to always do better.

My thanks to all those who helped me begin and continue my climb from the lowest places: Jimmy for his initial love, help, and support, and the members of my anonymous support group who gave me brotherhood (and sisterhood), advice, and a judgement-free space in which I was safe to open myself up completely so that true healing could begin. I love you all.

To anyone whom I have ever called "friend": I can't list all of you, because I know I would forget some people, but I love you all as well.

To my shmoo ... my Baby Doll ... my Lucky Charms ... you know I almost always don’t have the correct words to express my feelings, but I hope that you always know these feelings anyway. You helped me complete my climb by trying the crazy idea of loving and accepting me as I am, warts and all. It is truly humbling, and I hope that I have loved you the same way. Sure, I win at the game of "creating a dissertation," but you win at everything else!

And all the Glory to my Father in Heaven. If a middle-aged, barely-mathematically-literate goofball who spent his formative years studying music could actually survive graduate school, earn a master's degree, gain admission to a Ph.D. program, and successfully complete this dissertation, than I guess I can do all things ${ }^{1}$, indeed!

\footnotetext{
${ }^{1}$ Referencing Philippians 4:13: I can do all things through Christ who strengthens me.
} 


\section{TABLE OF CONTENTS}

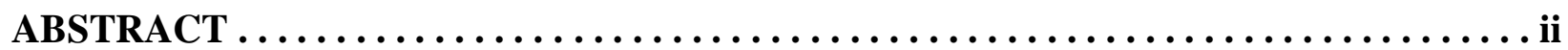

DEDICATION $\ldots \ldots \ldots \ldots \ldots \ldots \ldots \ldots \ldots \ldots \ldots \ldots \ldots \ldots \ldots \ldots \ldots \ldots$ iii

ACKNOWLEDGEMENTS $\ldots \ldots \ldots \ldots \ldots \ldots \ldots \ldots \ldots \ldots \ldots \ldots \ldots \ldots$ iv

LIST OF TABLES $\ldots \ldots \ldots \ldots \ldots \ldots \ldots \ldots \ldots \ldots \ldots \ldots \ldots \ldots \ldots \ldots \ldots \ldots \ldots \ldots$ ix

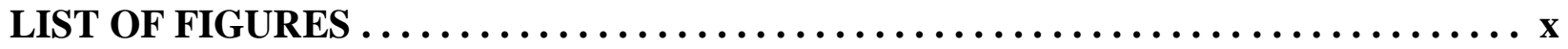

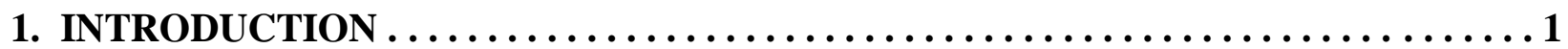

Research Questions .......................................

2. LITERATURE REVIEW $\ldots \ldots \ldots \ldots \ldots \ldots \ldots \ldots \ldots \ldots \ldots \ldots \ldots \ldots \ldots \ldots$

Infinitesimal-Based and Limit-Based Calculus: A Brief History $\ldots \ldots \ldots \ldots \ldots \ldots \ldots .4$

Some Conceptualizations of Differentials Found in the Literature $\ldots \ldots \ldots \ldots \ldots \ldots 6$

Differentials in Definite Integrals ..........................6

Differentials in Leibniz Derivative Notation .................... 9

Other Contexts ....................................11

Differences in "Mathematics" and "Physics" differentials ..........11

Notable conceptualizations in some textbooks ................14

Differentials as algorithmic tools .................... 17

Differential Conceptualizations in Randomly-Selected Textbooks .............17

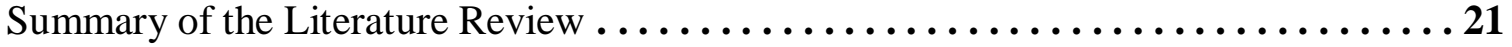

3. THEORETICAL PERSPECTIVE $\ldots \ldots \ldots \ldots \ldots \ldots \ldots \ldots \ldots \ldots \ldots \ldots \ldots \ldots \ldots$

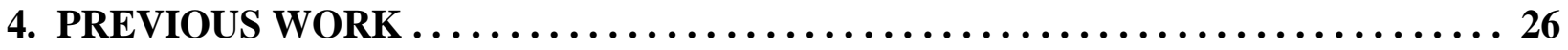

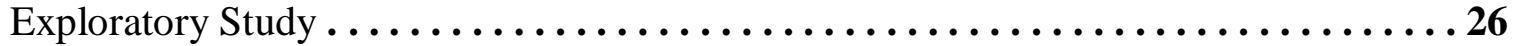

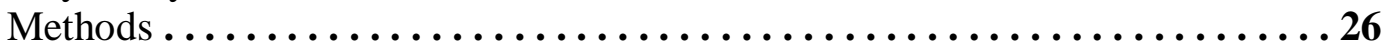

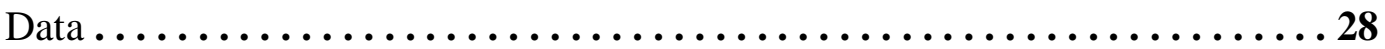

Results .............................................

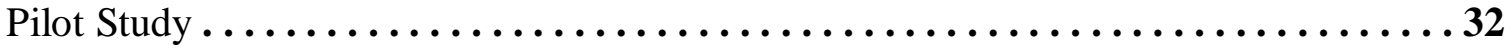

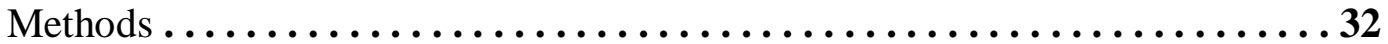

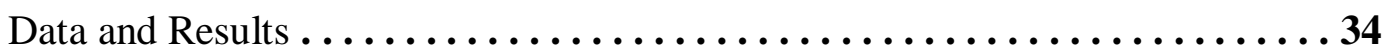

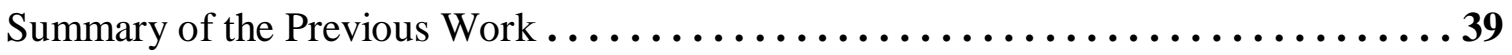

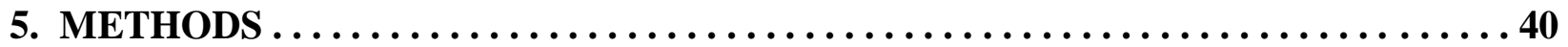

Data Collection ..........................................40

Interview subjects and consent $\ldots \ldots \ldots \ldots \ldots \ldots \ldots \ldots \ldots \ldots \ldots \ldots \ldots \ldots$

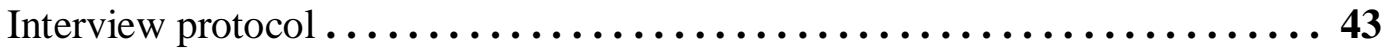

Introductory questions $\ldots \ldots \ldots \ldots \ldots \ldots \ldots \ldots \ldots \ldots \ldots \ldots \ldots, 43$

The questions containing differentials $\ldots \ldots \ldots \ldots \ldots \ldots \ldots \ldots \ldots . \ldots 44$

Two ancillary sets of questions $\ldots \ldots \ldots \ldots \ldots \ldots \ldots \ldots \ldots \ldots .45$ 


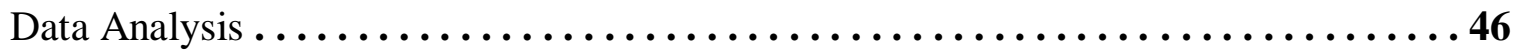

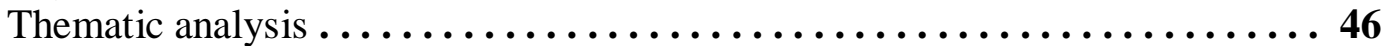

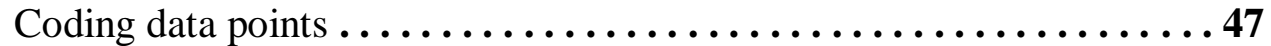

Data analysis for the second round of interviews $\ldots \ldots \ldots \ldots \ldots \ldots .54$

Visual Representations of Data .............................56

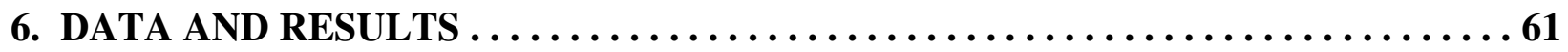

The Flowchart and the Final Tier 1 Themes $\ldots \ldots \ldots \ldots \ldots \ldots \ldots \ldots \ldots \ldots \ldots .61$

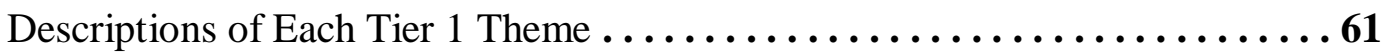

Themes N.4 and L. $4 \ldots \ldots \ldots \ldots \ldots \ldots \ldots \ldots \ldots \ldots \ldots \ldots, 63$

Themes N.4a and L.4a..........................63

Themes N.4b and L.4b............................6 64

Themes N.4c and L.4c .............................64

Themes N.3 and L. $3 \ldots \ldots \ldots \ldots \ldots \ldots \ldots \ldots \ldots \ldots \ldots . \ldots . \ldots 65$

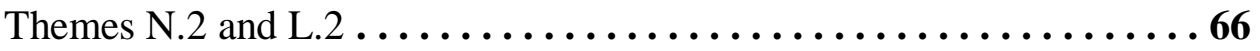

Themes N.1 and L.1 ...........................67

Themes N.1a and L.1a...........................6 67

Themes N.1b and L.1b..........................68

Themes N.1c and L.1c ............................69

Theme L.1d ..................................69

A brief note about subthemes ...................... 70

Descriptions and examples of each Tier 2 theme and subtheme $\ldots \ldots \ldots \ldots \ldots \ldots \ldots .70$

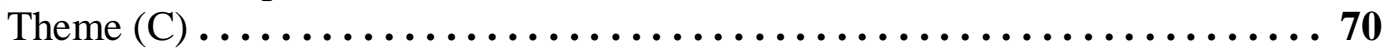

Theme (C).a........................... 71

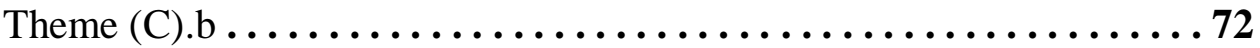

Theme (C).c.................................

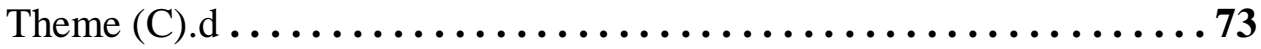

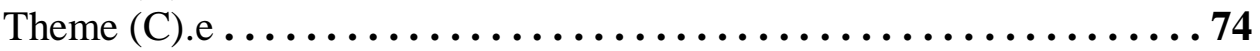

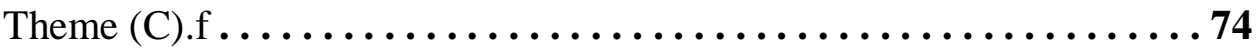

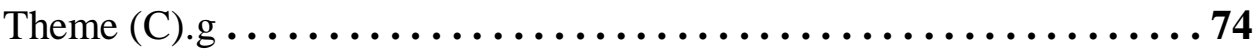

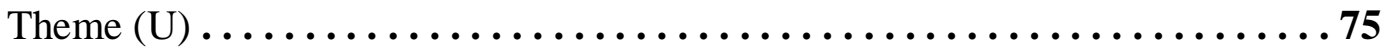

Theme (U).a................................. 75

Theme (U).b................................ 75

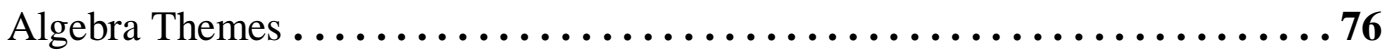

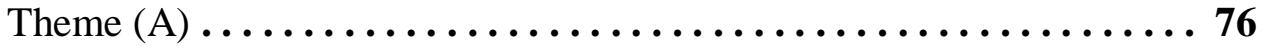

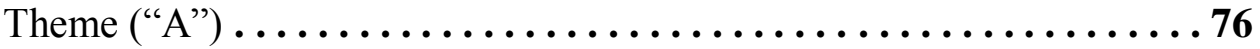

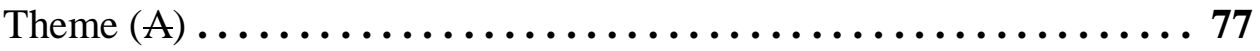

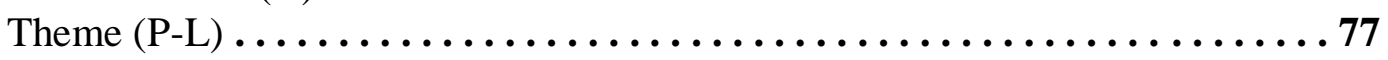

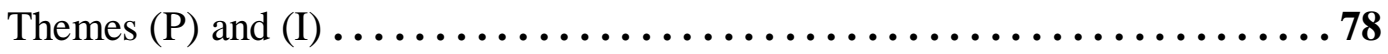

Summaries of each Interview Subject $\ldots \ldots \ldots \ldots \ldots \ldots \ldots \ldots \ldots \ldots \ldots \ldots \ldots \ldots . \ldots 78$

André .............................................79

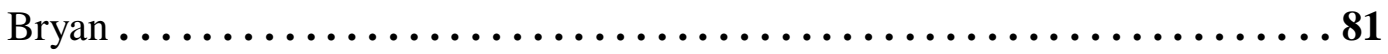

Christopher................................... 84

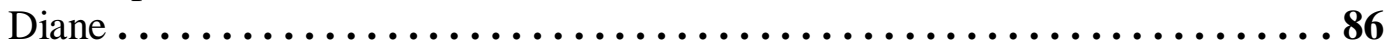

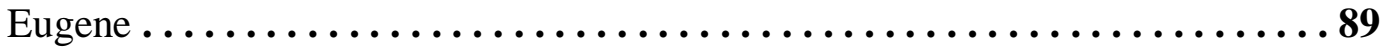

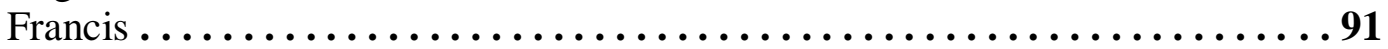


Gustav ................................... 93

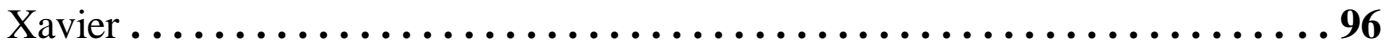

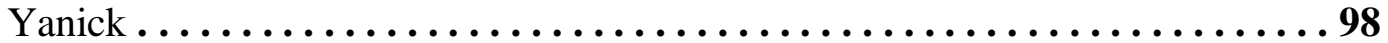

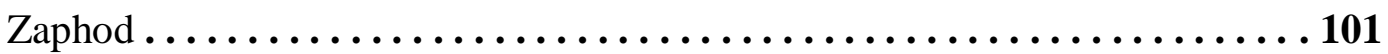

Summaries of Each Expression $\ldots \ldots \ldots \ldots \ldots \ldots \ldots \ldots \ldots \ldots \ldots \ldots \ldots \ldots \ldots \ldots$

The Initial "Differential" Question $\ldots \ldots \ldots \ldots \ldots \ldots \ldots \ldots \ldots \ldots \ldots \ldots . \ldots \ldots$

The Interview Subjects" "Small" Phrase ..................... 106

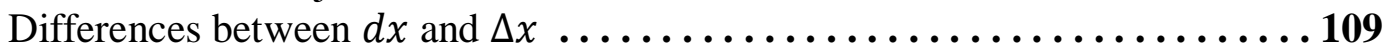

Leibniz Derivative Notation $\frac{d y}{d x} \ldots \ldots \ldots \ldots \ldots \ldots \ldots \ldots \ldots \ldots \ldots \ldots \ldots$

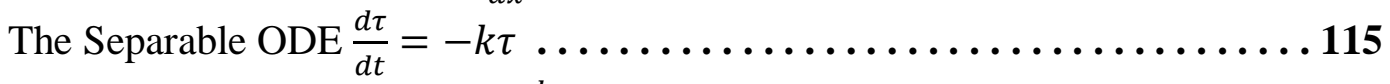

The Generic Definite Integral $\int_{a}^{b} f(x) d x \ldots \ldots \ldots \ldots \ldots \ldots \ldots \ldots \ldots \ldots$

The "Work" Problem Containing the Integral $\int_{0}^{50} 700-3 x d x \ldots \ldots \ldots \ldots 121$

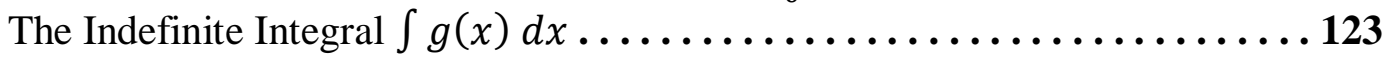

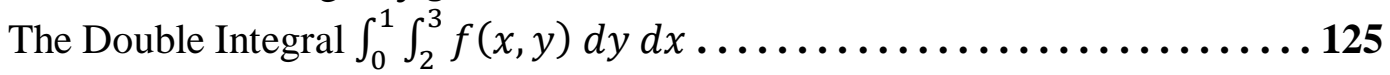

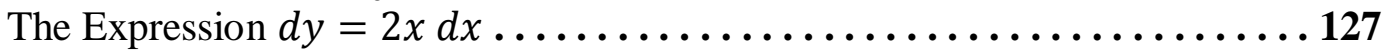

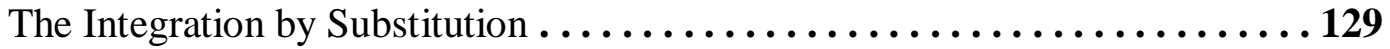

Answering the Research Questions ........................... 131

Question \#1 (How Extensive Is Each Concept Image) ............. 131

Question \#2 (Consistency within Each Individual) . . . . . . . . . . . . 134

Question \#3 (Consistency among All Individuals) .............. 136

Summary of the Data and Results ............................. 137

7. DISCUSSION ...................................... 139

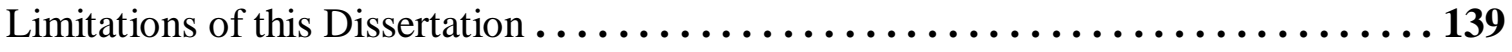

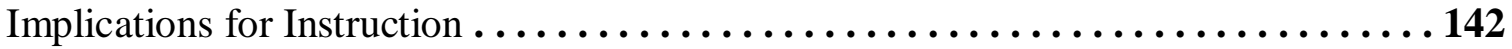

Possible Future Research . ............................. 148

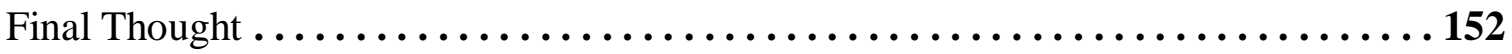

REFERENCES $\ldots \ldots \ldots \ldots \ldots \ldots \ldots \ldots \ldots \ldots \ldots \ldots \ldots \ldots \ldots \ldots \ldots \ldots \ldots \ldots$

APPENDIX A: Exploratory Study Protocol $\ldots \ldots \ldots \ldots \ldots \ldots \ldots \ldots \ldots \ldots \ldots$

APPENDIX B: Pilot Study Protocol $\ldots \ldots \ldots \ldots \ldots \ldots \ldots \ldots \ldots \ldots \ldots \ldots \ldots$

APPENDIX C: Consent Form for the Dissertation Study . . . . . . . . . . . . . 164

APPENDIX D: Dissertation Study Protocol $\ldots \ldots \ldots \ldots \ldots \ldots \ldots \ldots \ldots \ldots \ldots$ 


\section{LIST OF TABLES}

TABLE 1. EXPLORATORY STUDY'S PROTOCOL EXPRESSIONS $\ldots \ldots \ldots \ldots \ldots \ldots \ldots \ldots \ldots \ldots . . .27$

TABLE 2. SUMMARY OF THE PILOT STUDY'S PROTOCOL $\ldots \ldots \ldots \ldots \ldots \ldots \ldots \ldots \ldots \ldots \ldots \ldots$

TABLE 3. SUMMARY OF SUBJECT VIEWS AND PREFERRED STUDENT VIEWS OF DIFFERENTIALS ... 38

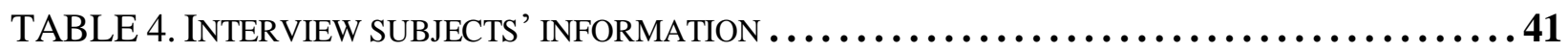

TABLE 5. LIST OF ALL TIER 2 THEMES FOUND IN MY WORK $\ldots \ldots \ldots \ldots \ldots \ldots \ldots \ldots \ldots \ldots .71$

TABLE 6. THE EXPRESSIONS IN WHICH $\Delta x$ WAS FiRST MENTIONED ................. 109

TABLE 7. SUMMARY OF INTERVIEW SUBJECTS' VIEWS OF THE DIFFERENTIALS IN $\frac{d y}{d x} \ldots \ldots \ldots \ldots 11$

TABLE 8. SUMMARY OF INTERVIEW SUBJECTS' VIEWS OF THE DIFFERENTIALS IN $\int_{a}^{b} f(x) d x \ldots 119$

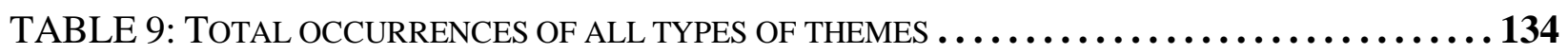

TABLE 10. Some ExAMPLES OF THE ALgEBRA THEMES $\ldots \ldots \ldots \ldots \ldots \ldots \ldots \ldots \ldots \ldots \ldots$ 


\section{LIST OF FIGURES}

FIGURE 1. VON KORFF AND REBELLO'S DEFINITE INTEGRATION FRAMEWORK $\ldots . \ldots \ldots \ldots \ldots . .8$

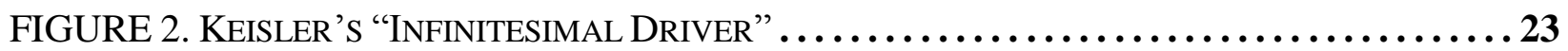

FIGURE 3. TWO EXAMPLES OF THE SELECTION OF DATA POINTS FROM ANDRÉ'S INTERVIEW . . . 49

FIGURE 4. AN EXCERPT FROM MY THEMATIC MAP $\ldots \ldots \ldots \ldots \ldots \ldots \ldots \ldots \ldots \ldots \ldots \ldots . . \ldots 50$

FIGURE 5. THE INITIAL LIST OF THEMES (FROM THE THEMATIC MAP) $\ldots \ldots \ldots \ldots \ldots \ldots \ldots . .51$

FIGURE 6 . THE FIRST ATTEMPT AT A FINAL LIST OF THEMES $\ldots \ldots \ldots \ldots \ldots \ldots \ldots \ldots \ldots \ldots . \ldots 2$

FIGURE 7. THE FLOWCHART CONTAINING ALL TIER 1 THEMES $\ldots \ldots \ldots \ldots \ldots \ldots \ldots \ldots \ldots . \ldots 55$

FIGURE 8 . AN EXAMPLE OF AN INDIVIDUAL FLOWCHART $\ldots \ldots \ldots \ldots \ldots \ldots \ldots \ldots \ldots \ldots \ldots . \ldots 57$

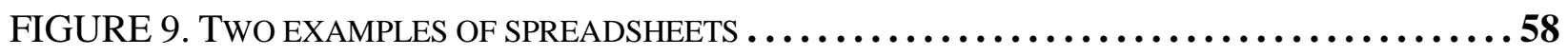

FIGURE 10. THE FLOWCHART CONTAINING ALL TIER 1 THEMES (RE-PRESENTED).$\ldots \ldots \ldots \ldots .62$

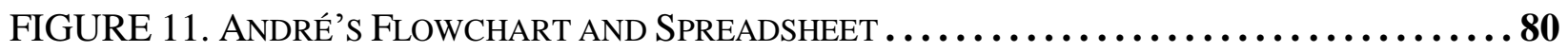

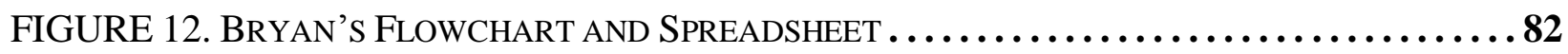

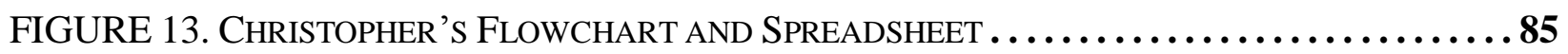

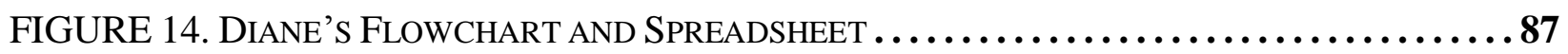

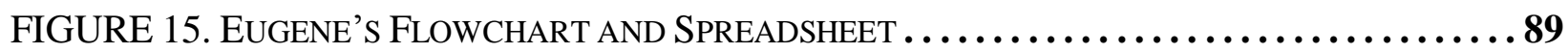

FIGURE 16. FRANCIS' FlowCHART AND SPREADSHEET $\ldots . \ldots \ldots \ldots \ldots \ldots \ldots \ldots \ldots \ldots . . \ldots 92$

FIGURE 17. GUSTAV's FlOWCHART AND SPREADSHEET $\ldots \ldots \ldots \ldots \ldots \ldots \ldots \ldots \ldots \ldots \ldots . . \ldots 4$

FIGURE 18. XAVIER'S FlOWCHART AND SPREADSHEET $\ldots \ldots \ldots \ldots \ldots \ldots \ldots \ldots \ldots \ldots \ldots . . \ldots 7$

FIGURE 19. YANICK's FLOWCHART AND SPREADSHEET $. . \ldots \ldots \ldots \ldots \ldots \ldots \ldots . \ldots . \ldots 99$

FIGURE 20. ZAPHOD's FLOWCHART AND SPREADSHEET $\ldots \ldots \ldots \ldots \ldots \ldots \ldots \ldots \ldots \ldots \ldots . \ldots 102$

FIGURE 21. FLOWCHART AND SPREADSHEET FOR THE "DIFFERENTIAL" QUESTION $. . \ldots \ldots \ldots . .105$ 
FIGURE 22. FlowCHART AND SPREADSHEET FOR THE "PHRASE" QUESTION $\ldots \ldots \ldots \ldots \ldots \ldots \ldots 1$

FIGURE 23. FLOWCHART AND SPREADSHEET FOR THE "DELTA" QUESTION $\ldots \ldots \ldots \ldots \ldots \ldots \ldots 110$

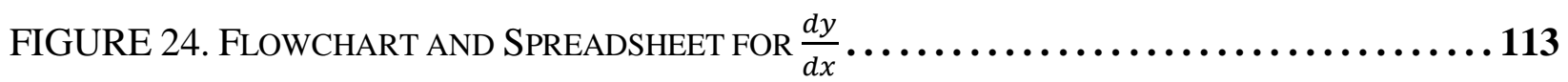

FIGURE 25. FLOWCHART AND SPREADSHEET FOR THE SEPARABLE ODE $\ldots \ldots \ldots \ldots \ldots \ldots \ldots 116$

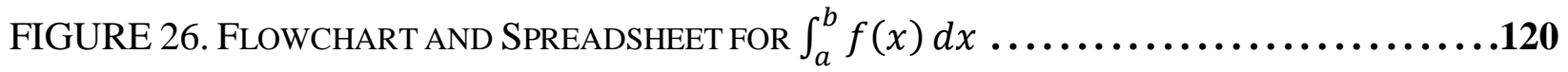

FIGURE 27. FLOWCHART AND SPREADSHEET FOR THE "WORK" PROBLEM $\ldots \ldots \ldots \ldots \ldots \ldots . . \ldots 122$

FIGURE 28. FLOWCHART AND SPREADSHEET FOR $\int g(x) d x \ldots \ldots \ldots \ldots \ldots . . . \ldots \ldots . . . . .124$

FIGURE 29. FLOWCHART AND SPREADSHEET FOR $\int_{0}^{1} \int_{2}^{3} f(x, y) d y d x \ldots \ldots \ldots \ldots \ldots \ldots \ldots . \ldots 126$

FIGURE 30. FLOWCHART AND SPREADSHEET FOR $d y=2 x d x \ldots \ldots \ldots \ldots \ldots \ldots \ldots \ldots \ldots$

FIGURE 31. FLOWCHART AND SPREADSHEET FOR THE INTEGRATION BY SUBSTITUTION ........130

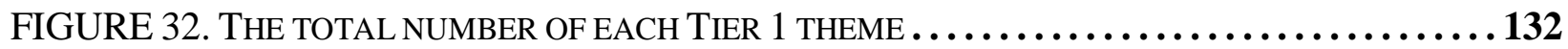

FIGURE 33. THE TOTAL NUMBER OF EACH TIER 2 THEME $\ldots \ldots \ldots \ldots \ldots \ldots \ldots \ldots \ldots \ldots \ldots . \ldots 133$ 


\section{INTRODUCTION}

The differential, symbolized by a $d$ and a second letter usually depending on context (some examples are $d x, d t$, and $d A)^{2}$ is a symbol that is found in various mathematical situations, including:

- Expressions for integration, as in $\int_{a}^{b} f(x) d x$ and $\int g(x) d x$.

- A possible notation for a derivative, as in the differential equation $\frac{d P}{d t}=3 P$

- Relationships with other differentials, as in the evaluation of the integral $\int_{1}^{5} 2 x \sin \left(x^{2}\right) d x$, which can be evaluated by letting $u=x^{2}$ and using the relationship $d u=2 x d x$.

The notations used in these situations are well-known and common, but that does not mean that the interpretations of the differentials in these notations will be universal. Specifically, one might ask the following questions about the differentials in the above examples and get different answers from different people:

- How do you think about the " $d x$ " in a definite integral? Is it some referent to a Riemann sum's $\Delta x$, some sort of infinitesimal amount, or only an indicator of the variable of integration? Is the same true for the " $d x$ " in an indefinite integral?

- How do you think about the notation " $\frac{d P}{d t}$,? Is it merely a notation that means "the derivative of $P$ with respect to $t$, , or is it an actual ratio between two specific terms: " $d P$ " and " $d t$ "?

- When performing integration by substitution, is the step " $d u=2 x d x$ " a representation of the local linearity of the curve $u=x^{2}$ in a very small neighborhood around $x$ ? Is it merely an algebraic manipulation of the idea that $\frac{d u}{d x}=2 x$ ? Is it both of these things? Neither?

\footnotetext{
${ }^{2}$ Throughout this dissertation, I shall use the default notation " $d x$ " when discussing a general differential or a differential without context.
} 
Historically, the notations $\int_{a}^{b} f(x) d x, \frac{d P}{d t}$, and $d u=2 x d x$ were to represent, respectively, the sum of infinitely many infinitesimal quantities $f(x) d x$, a ratio between two infinitesimal quantities $d P$ and $d t$, and an infinitesimal change in one quantity given an infinitesimal change in another (Ely \& Boester, 2010; Keisler, 2012). However, these initial intents may have been muddled by the abandonment of the infinitesimal-based calculus of Leibniz that gave us these notations and the introduction and acceptance of a newer calculus that kept the same notations but was based around "epsilon-delta" and limits rather than infinitesimals. Given that there exist infinitesimal-based and limit-based treatments of calculus that use the same differential-containing notations, it is perhaps not surprising that various ways to interpret differentials have arisen, and it is possible that an individual might not have a simple, clear answer to the question "What is the meaning behind the symbol " $d x$ ”? As David Tall writes:

$\frac{d y}{d x}$ proves to be almost indispensable in the calculus. Yet it causes serious conceptual problems. Is it a fraction, or a single indivisible symbol? What is the relationship between the $d x$ in $\frac{d y}{d x}$ and the $d x$ in $\int f(x) d x$ ? Can the $d u$ be cancelled in the equation $\frac{d y}{d x}=\frac{d y}{d u} \frac{d u}{d x}$ ? Giving a modern meaning to these terms that allows a consistent meaningful interpretation for all contexts in the calculus is possible but not universally recognized. On the other hand, failing to give a satisfactory coherent meaning leads to cognitive conflict which is usually resolved by keeping the various meanings of the differential in separate compartments $\left(\frac{d y}{d x}=\lim _{\Delta x \rightarrow 0} \frac{\Delta y}{\Delta x}\right.$ in differentiation, and $d x$ means "with respect to $x$ " in integration). (Tall, 1993, p. 6) 
Some mathematicians might have compartmentalized their interpretation of the differential in a way similar to what Tall describes. It is also possible that some mathematicians might have found a consistent, meaningful interpretation of the differential no matter in what context the differential is presented. But even if this is so, there is no guarantee that one mathematician's consistent, meaningful interpretation will be the same as his or her colleagues' consistent, meaningful interpretations, just as there is no guarantee that all mathematicians who compartmentalize their interpretations of differentials will do so in the same way. This dissertation will analyze the "serious conceptual problems" suggested by Tall's quote by exploring how mathematicians think about differentials that are presented to them in various contexts. Not only will how interview subjects conceptualize these various differentials be analyzed, but also how consistent and universal their particular conceptualizations are.

\section{Research Questions}

Thus, this research will answer the following questions:

1. How extensive are the concept images of differentials held by expert mathematicians?

2. What levels of consistency, if any, exist in the concept images of the differential within each individual?

3. What levels of consistency, if any, exist in the concept images of the differential among all mathematicians interviewed? 


\section{LITERATURE REVIEW}

I have found no research that specifically addresses expert conceptualizations of differentials. There are a few articles I will present that discuss expert conceptualizations, but none of which are based on research. There are research studies involving conceptualizations of differentials, but these studies primarily focus on student conceptualizations of definite integrals, with any discussion of differentials being secondary. In this section, I will briefly discuss the history of calculus notation as it pertains to differentials, review all the literature I have found and its relevance to my topic, and close with the differential conceptualizations I found in two surveys of mathematics textbooks.

\section{Infinitesimal-Based and Limit-Based Calculus: A Brief History}

Gottfried Leibniz and Isaac Newton are said to have developed calculus by laying out unified theories of differentiation and integration, even though they differed in their interpretations of the mathematical constructs behind these theories. Newton had views that were more fluid than Leibniz'. For example, he defined the derivative of a function at various times using infinitesimals, limits, or velocities (Keisler, 2012), whereas Leibniz favored the use of infinitesimals, which were informally defined as numbers greater than zero but smaller than any real number (Ely, 2010; Henry, 2010). For Leibniz, these infinitesimals were thought of as "ideal" elements, like complex numbers, and just as complex numbers could be used to show otherwise inaccessible mathematical truths, infinitesimals could be used to show otherwise inaccessible ideas that mathematicians understood intuitively (Keisler, 2012). Leibniz' notations were meant to suggest this infinitesimal-

based approach, with $\frac{d y}{d x}$ suggesting a ratio of infinitesimal $d y$ and $d x$ and $\int_{a}^{b} f(x) d x$ suggesting an infinite sum of products with finite factor $f(x)$ and infinitesimal amount $d x$ (Ely \& Boester, 2010; Keisler, 2012). 
However, Leibniz and his followers did not generate a formal logical system surrounding these infinitesimals, which led to apparent inconsistencies within his infinitesimal-based approach to calculus. Examples of these inconsistencies include some ambiguity as to when infinitesimals should be considered numerical entities and when they should be disregarded as nonentities, and whether the Archimedean principle ${ }^{3}$, which applies to real numbers, also applies to infinitesimal numbers (Martínez-Torregrosa, López-Gay, \& Gras-Martí, 2006; Robinson, 1966). The steps toward a more-recognizable modern Calculus began in 1821, with Cauchy's definitions of integration and differentiation in terms of limits. Cauchy still used infinitely small quantities in his work, viewing them as a foundation for the theory of limits, but regarded them as variables whose limits approached zero instead of as static infinitesimals (Robinson, 1966). This view of infinitesimals eliminated many of the inconsistencies found in Leibniz' work (MartínezTorregrosa, López-Gay, \& Gras-Martí, 2006; Robinson, 1966), but it took a precise definition of the real number system, Cantor's development of set theory, and Weierstrass' introduction of the epsilon-delta condition before limits became the rigorous basis of standard analysis (Keisler, 2012).

Keisler (2012) suggests that infinitesimals have greater intuitive appeal, but notes that the reason why the limit approach to Calculus was widely adopted was because it was the first logically-consistent system found. But just as limits did not come into their own without the aid of developments in real numbers and set theory, so too would it take other advancements in mathematical logic and language before infinitesimal-based calculus could be formalized and all of its logical inconsistencies eliminated. Specifically, Gödel's Completeness Theorem and Skolem's construction of the hyperreal integers were tools that enabled Abraham Robinson to

\footnotetext{
${ }^{3}$ For any $0<a<b$, there exists $n \in \mathbb{N}$ such that $a n>b$.
} 
prove that infinitesimals were actually based in mathematical logic and develop infinitesimalbased nonstandard analysis (Keisler, 2012; Vinsonhaler, 2016). After the publication of Robinson's Non-standard Analysis in 1966, a small number of textbooks were written for the teaching of this infinitesimal-based calculus, including ones from Keisler (2012) and Henle and Kleinberg (2003).

\section{Some Conceptualizations of Differentials Found in the Literature}

I have found little literature in which differentials are the main topic of discussion. Thus, most of the literature that I have found that addresses conceptualizations of differentials does so in order to achieve some specific purpose. Some of these purposes include analyzing differentials as a smaller part of definite integration and Leibniz derivative notations, discussing some differences between "mathematics" and "physics" conceptualizations of differentials, and proposing possible differential conceptualizations to the reader. To account for these different purposes, I will partition the discussions of the literature I have found into multiple sections.

\section{Differentials in Definite Integrals}

In Physics Education Research (PER) literature, a common conception of the definite integral's differential is that it represents a "small" amount of some element of the situation modeled by the integral (e.g. Hu \& Rebello, 2013; Nguyen \& Rebello, 2011; Sealey \& Thompson, 2016). This is particularly emphasized in Nguyen and Rebello (2011), who found that, while students could identify when integration was needed to solve a problem, they had difficulty interpreting the differential element of the integral as an infinitesimal amount, which Nguyen and Rebello found to be an important part of solving integral problems. They specifically noted the importance of viewing the $d x$ as a small amount, rather than as a difference between two $x$-values. In contrast, Sealey and Thompson (2016) noted the importance of viewing the $d x$ additionally as 
the difference between two $x$-values, allowing for the $d x$ to be either positive or negative, depending on the direction of integration. For example, in the integral $\int_{a}^{b} f(x) d x$ in which the lower limit of integration is greater than the upper limit, Sealey and Thompson claimed that viewing the $d x$ as a negative quantity was helpful in conceptualizing the meaning of this "backward" integral.

The importance of viewing the differential as a small amount seems to extend into the notation and development of the definite integral. Sealey's $(2008 ; 2014)$ framework of the definite integral defined the $\Delta x$ as a small amount or change, but Von Korff and Rebello's (2012) paper gave a definite integral framework similar to Sealey's framework with the addition of another row of objects that contains the infinitesimal $d x$ rather than Sealey's $\Delta x$ (For example, the product $\Delta x=v \Delta t$ is replaced with the infinitesimal product $d x=v d t$. See Figure 1.) By including these additional objects in their framework, they ascribed a meaning to the definite integral's $d x$ : it is its own entity and important to the understanding of integration and not merely notation that is used once the limit process has been completed.

Artigue (1991) described two main categories of responses which were categorized as "two poles" between which other conceptualizations resided. At one end, students claimed that differentials merely served to signify the variable of integration, and at the other end, the differentials represented a small amount of some physical quantity. Examples of student responses that describe both ends are "To integrate [a function in terms of $l$ ], it is essential not to think about what $d l$ represents, but to proceed mechanically, otherwise we are done for," and "In fact, it does not matter at all, when integrating, $d l$ becomes a variable of integration" (Artigue, Menigaux, and Viennot, 1990, p.264) for the first and " $d l$ is a small length" and "little bit of wire" (Artigue, Menigaux, and Viennot, 1990, p.264) for the second. Other responses that were noted as being 


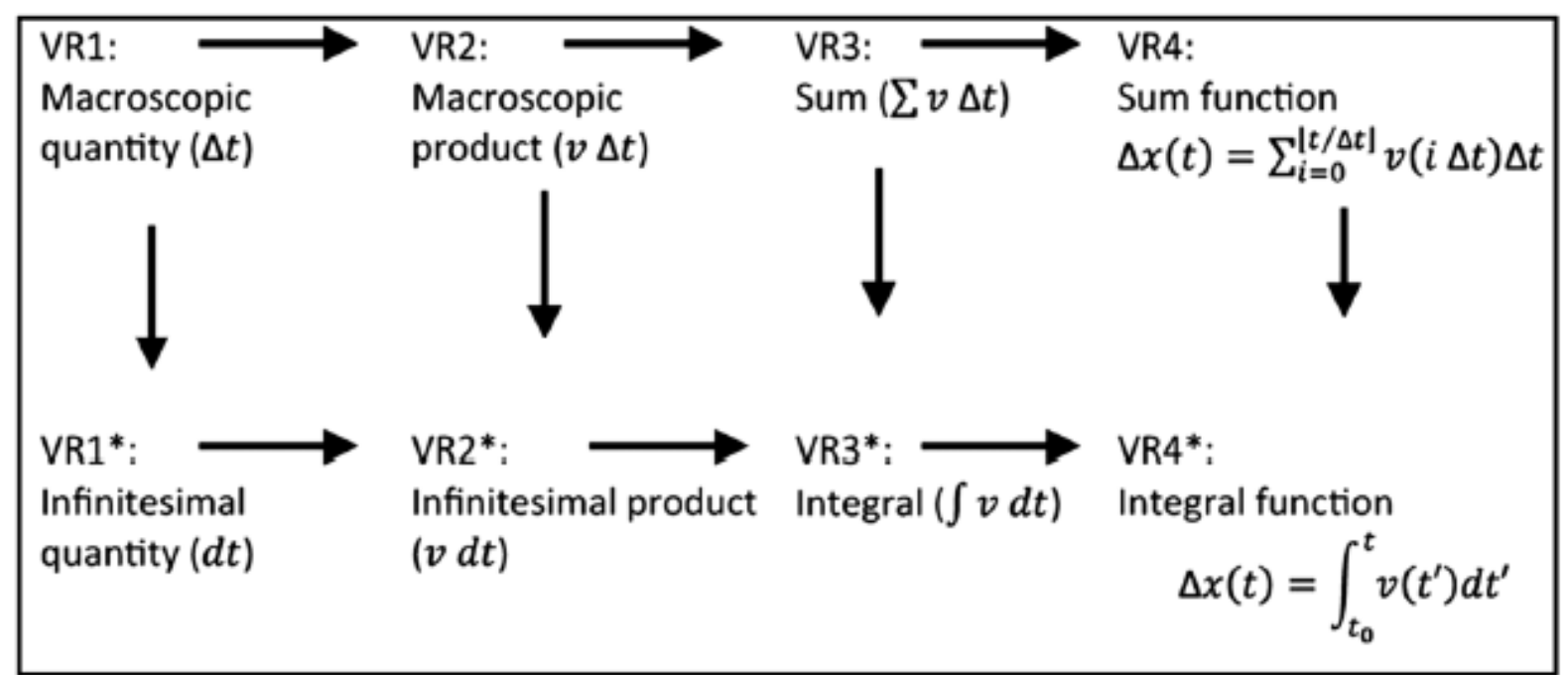

Figure 1: Von Korff and Rebello's definite integration framework (Von Korff and Rebello, 2012, p.3)

within these two ends include a differential as "an infinitely small element," and $d z=\lim _{\Delta z \rightarrow 0} \Delta z$ (Artigue, 1991). As will be seen later in this dissertation, Artigue's example of a continuum of responses contained within two well-defined ends will serve as a model for my own differential conceptualization framework.

Additionally, Jones' (2015) showed three common conceptualizations that some students have for the differential in a definite integral. Students were interviewed using a protocol that aimed to categorize and analyze students' symbolic forms of the definite integral. Symbolic forms will be described in Chapter 7 of this dissertation, while how the differential in each form was conceptualized is described here.

One form was the function matching symbolic form, in which the integrand is interpreted as the derivative of an unknown function, with the differential stating the variable by which that function was differentiated (Jones, 2015). Thus, when seeing the integral $\int_{0}^{2} x^{2} d x$, the task is to determine what function yields $x^{2}$ when differentiated in terms of $x$. I would consider this form to be similar to the "variable indicator" forms mentioned in the other two sections. Another form was the perimeter and area symbolic form, in which every part of the definite integral symbol defines 
the boundary of a particular shape (Jones, 2015). The differential specifically denotes one of the "sides" of that shape: in a first-quadrant shape, $d x$ would denote that the bottom of the shape was the $x$-axis while $d y$ would denote that the left side of the shape was the $y$-axis. This seems a variation on the "variable indicator" themes, as this theme points to variables in a geometric manner, rather than the more algorithmic manner of the other "variable indicator" themes.

The other symbolic form was the adding up pieces form, which, similar to a Riemann sum, is meant to emphasize the multiplicative-based summation of a definite integral. In this form, the differential represents an "infinitesimally small" amount of the domain of integration (Jones, 2015). Unlike Oehrtman's (2009) “collapsing metaphors," in which slices of the area under a curve are viewed as collapsing to one-dimensional segments after a limit process, Jones noted that many of his students viewed their "infinitesimally thin" rectangles as still having two dimensions. Thus, this differential retains a "concreteness" that a collapsed differential might lack.

\section{Differentials in Leibniz Derivative Notation}

Compared to the relatively robust literature I found that addressed the differentials in definite integration, there were fewer papers that addressed the differentials in Leibniz derivative notation. Unlike definite integrals, whose notation always includes a differential, derivative

notation can be expressed with not only the differential-containing " $\frac{d y}{d x}$ ”, but also the common, differential-free $f^{\prime}(x)$. Thus, discussions of derivatives might not as naturally lend themselves to discussion of differentials.

Zandieh's (2000) derivative framework, which is used to analyze how students conceptualized the derivative, is a commonly referenced piece of mathematics education literature. Her framework consists of three layers of what she called process-object pairs, based on her understanding that each layer can both be viewed as a dynamic process as well as a static object. 
To give the example relevant to this dissertation, calculating the numerator and denominator in the average rate of change is a process that can be represented by the object $\frac{\Delta y}{\Delta x}$. The limiting process applied to this ratio is the process of analyzing multiple average rates of change as $\Delta x$ goes to zero, and is represented by both $\lim _{\Delta x \rightarrow 0} \frac{\Delta y}{\Delta x}$ and the more compact $\frac{d y}{d x}$. While students' conceptualizations of the $\Delta y$ and $\Delta x$ are discussed in her paper, Zandieh does not discuss students' conceptualizations of the individual $d y$ and $d x$ in the notation $\frac{d y}{d x}$. This perhaps suggests that the idea that, in this framework, defining the individual $d y$ and $d x$ is not conducive to a proper understanding of derivative. When Roundy, Dray, Manogue, Wagner, and Weber (2015) extended Zandieh's "physical" context to include examples whose dependent variable was not time, the example they gave described the instantaneous rate of change of the volume of a cylinder with respect to pressure with the notation $\frac{d V}{d p}$, perhaps suggesting that the Leibniz differential-based notation is more suitable for physical representations.

I mention two other papers, one that directly addressed the differentials in Leibniz derivative notation, and the other from which a differential conceptualization can only be implied. First, in a paper that proposed an interpretation of $\frac{d y}{d x}$, Thurston (1972) argued that $\frac{d y}{d x}$ should be translated as $\frac{y^{\prime}(\tau)}{x^{\prime}(\tau)}$, where the numerator and denominator of $\frac{d y}{d x}$ each indicate a differentiated function of another variable. This translation suggests that, for Thurston, the " $d$ " in each differential suggests the act of differentiation. Second, Orton (1983) interviewed sixty high school and fifty college students majoring in mathematics education, asking them questions regarding rate of change, differentiation, and applications. Only one of the tasks in his paper regarded differentials directly: it asked the interview subjects to define $\delta x, \delta y, \frac{\delta y}{\delta x}, d x, d y$, and $\frac{d y}{d x}$. When 
describing the results, Orton noted many misunderstandings with the differential definitions, but said that "This was expected in the sense that the symbols are not really meaningful except when used together as $\frac{d y}{d x}$ or when used in integration" (p.240). Since there exist conceptualizations that disagree with the idea that the individual differentials in $\frac{d y}{d x}$ are "not really meaningful," I would suggest that Orton personally adopted the conceptualization that these differentials are merely notation.

\section{Other Contexts}

In this section, I will summarize conceptualizations I found that do not specifically address differentials in definite integral or derivative notations. Instead, the literature cited in this section categorized lists of conceptualizations, compared "physics" differentials with "mathematics" differentials, or discussed uncommon ways to utilize differentials in calculus.

Differences in "Mathematics" and "Physics" differentials. The first two works in this section listed conceptualizations found in physics research. The others provided their particular comparisons, some grand and some small, of how differentials are viewed in physics and mathematics, an idea that will also manifest in my research.

$\mathrm{Hu}$ and Rebello (2013) discussed student uses of differentials in physics problems by conducting group interviews with thirteen students taking a calculus-based physics course. From these interviews, Hu and Rebello identified four mathematical resources about differentials. The first and fourth of these resources match Artigue's two "poles" described above: thinking of a differential as a "small amount," also described as an "infinitesimal" amount, and thinking of the differential as merely an indicator of the variable of integration, with no physical meaning. The other resources are viewing the definite integral's differential as points on a line, and viewing the " $d$ " in a differential as a cue to take a derivative. In addition to these differential 
conceptualizations, $\mathrm{Hu}$ and Rebello also noted student conceptual metaphors that were used in the construction of integrals, each of which involved differentials. The objects metaphor relies on the idea of a differential as an object, the locations metaphor relies on viewing the differentials as a location in space, the machines metaphor relies on the concept of " $d$ " triggering differentiation, and the motion along a path metaphor relies on the second variable in the differential as the line upon which an imaginary traveler moves. In another paper, Martínez-Torregrosa, López-Gay, and Gras-Martí (2006) discussed the historical development of the differential in an attempt to provide a better conceptualization of the differential in modern physics courses. Thus, this paper discussed in turn each of the following: the informal infinitesimals of Leibniz, the differentials of Cauchy, which were viewed as variables who approach zero (and were thus a precursor to limits,) and the Fréchet differential (described below).

Many papers that I found described versions of what I will call a "mathematics versus physics" conceptualization divide. Some papers already mentioned in this section discussed such divides. Artigue's aforementioned works (Artigue, Menigaux, and Viennot, 1990; Artigue, 1991) were based on interviews of both mathematics and physics students. In the physics students' questionnaires, the responses consisted of the "two poles" described above. (Artigue, 1991), while in the mathematics students' questionnaires, there were differences between how students defined and used differentials. When defining differentials, mathematics students tended to use a "linear approximation" definition, but when using differentials, students tended to view them as parts of algorithmic process that are manipulated automatically (Artigue \& Viennot, 1987; Artigue, 1991). Similarly, Jones (2013) gave two interviews to his students, one "mathematics" interview and one "physics" interview, named for the curricula in which the particular interview items would be found. He analyzed his three symbolic forms in both mathematics and physics contexts, concluding 
that, while all three conceptualizations can be useful in dealing with integrals presented without any context, viewing the differential as an indicator for either a variable or a border is not productive for integrals within a physical context (Jones, 2015).

One paper that addressed mathematics and physics differentials directly came from LópezGay, Martínez Sáez, and Martínez Torregrosa (2015), in which they addressed the importance of differentials in physics classes. In doing so, they listed four conceptualizations used when teaching mathematics and four conceptualizations used when teaching physics. Two of the "mathematics" conceptualizations were already mentioned in this literature review: the differential given without any meaning, and the differential as a formally-defined nonstandard infinitesimal. The other two included the differential used in what I will call traditional linear approximation, in which $\Delta y \approx$ $d y=f^{\prime}(x) d x$ with $d x=\Delta x$, and the Fréchet differential, which is similar to the differential in traditional linear approximation, only having the additional characteristic that the difference $(\Delta y-d y)$ must be infinitely small in relation to $\Delta x$. Two of the four "physics" conceptualizations were also mentioned earlier: versions of the "meaningless" differential, and the differential as traditional linear approximation. The other two involve the differential $d y$ as infinitesimal increment equal to the infinitesimal $\Delta y$, and the differential $d y$ as an infinitesimal approximation to $\Delta y$. In both of these, the word "infinitesimal" is described as intuitive and not well-defined.

Other works mentioned a divide, but without discussing the differential directly. Dunn and Barbanel (2000) discussed how mathematicians and physicists would disagree on the development of a definite integral: mathematicians viewing it as the limit of a Riemann sum, with physicists viewing it as the sum of infinitely-many products of $f(x)$ and an infinitesimal $d x$. Thus, they imply that the "physics" differential has actual meaning in the integration symbol. Finally, Henry 
(2010), in her introduction, stated that mathematicians view differentials as approximating functions $\left(d y=f^{\prime}(x) d x\right)$ while physicists view them as "small quantities."

Notable conceptualizations in some textbooks. I have found one collection and two examples of textbooks in which the presentation of differentials and/or differential-based topics is atypical. First, there is a group of textbooks that teaches differentials as hyperreal infinitesimals as found in nonstandard analysis. To briefly explain: nonstandard analysis allows for the creation of a hyperreal number system, of which the standard real numbers are a subset. An infinitesimal, $\epsilon$, is defined as any hyperreal number with the condition $-a<\epsilon<a$ for every positive real number a. In such a system, every hyperreal noninfinite number is infinitely close to only one real number, and that real number is defined as the standard part of that hyperreal. With the addition of the Transfer Principle, which states that the extension of any real-valued function to a hyperrealvalued function has the same properties as the original function, operations and functions based in real numbers may be performed with hyperreals as well (Keisler, 2012). As mentioned above, Robinson's Non-standard Analysis (1966) introduced and formalized infinitesimals, but there are more recent textbooks such as Keisler (2012), Henle and Kleinberg (2003), Hrbacek, Lessmann, and O'Donovan (2014), and Sloughter (2007) that teach a first-year calculus based on these infinitesimal differentials.

While some of these books use different terms for similar ideas, such as Sloughter (2007) using the term "shadow" instead of standard part, and Hrbacek, Lessmann, and O'Donovan (2014) substituting "ultrasmall" and "observable" for "infinitesimal" and "real", each book adopts a conceptualization of the differential as a well-defined infinitesimal. With this conceptualization, the integral $\int_{a}^{b} f(x) d x$ is considered exactly as the notation presents it to be: an infinite sum of products of this formally-defined infinitesimal $d x$ with real-valued $f(x)$. The derivative is the 
standard part of the ratio of two formally-defined infinitesimal quantities, $\frac{d y}{d x}$ and since the standard part of a product of two numbers is equal to the product of the standard part of two numbers, the Chain Rule $\frac{d y}{d t}=\frac{d y}{d x} \cdot \frac{d x}{d t}$ can be thought of as literally the reducing or cancellation of fractions of infinitesimal quantities instead of a notational trick. Any other relationship between differentials, such as the example $d u=\frac{1}{2 \sqrt{t}} d t$, represents a formally-defined infinitesimal change in $t$ as a factor of a formally-defined infinitesimal change in $u$ (Ely \& Boester, 2010; Henle \& Kleinberg, 2003; Keisler, 2012).

Courant and John (1965) described differentials using the term physically infinitesimal, or finite, nonzero real values that are "smaller than the degree of accuracy required (Courant and John, 1965, p.184). This definition is motivated by their idea that in the natural world, one cannot measure an object precisely, since one can obtain only a degree of accuracy that is limited by the physical nature of the measuring instrument. Similarly, one could carry out any mathematical limiting process to a certain level of precision, but true exactness is nothing more than a "mathematical idealization" (p. 184). With this idea in mind, Courant and John defined physically infinitesimal quantities as finite nonzero, real values that are small enough for a specific task. Two examples they gave are "smaller than the fractional part of a wavelength" or "smaller than the distance between two electrons in an atom" (p. 184). Calculations carried out by using these types of values might not result in exact answers, but since exact answers are not possible in an imperfect physical world, these results are acceptable. Dray (2013) did not use the term "physically infinitesimal" in his paper, but described a physicist view of a differential as "being much smaller than the scale imposed by the physical situation, but not so small that quantum mechanics matters" (p.17), a similar notion to Courant and John's "physically infinitesimal". This type of infinitesimal alludes to Oehrtman's (2009) “physical limitations” metaphor found in some students' concepts 
of limits. Oehrtman's metaphor referenced an argument in which "there is a scale beyond which nothing can be observed, be measured, or perhaps even exist" (p. 418). While this metaphor can be troublesome for some, I believe that Courant and John's idea of physically infinitesimal allows for a differential conceptualization that might adhere to the spirit of this metaphor yet still be accessible. One can conceptualize a differential as a value so small as to be undetectable by any current measuring instrument. It would then be both a finite, real number, but also "nonexistent" in a sense. ${ }^{4}$

Finally, Thompson (1910), wrote a textbook that is centered on the idea of differentials as one of the primitive ideas that defines the whole of first-year calculus. As he stated in the introduction to his book:

The preliminary terror, which chokes off most fifth-form boys from even attempting to learn how to calculate, can be abolished once for all by simply stating what is the meaning - in common-sense terms - of the two principal symbols that are used in calculating. These dreadful symbols are:

(1) $d$, which merely means "a little bit of'....

(2) $\int$ which is merely a long S, and may be called. . . "the sum of." (Thompson, 1910, p.1)

He then defined differentiation and integration around the idea of the "indefinitely small" differentials. He did not give a formal definition for his "smallness", and, indeed, used many synonyms to describe it: beginning the book by stating that, throughout all calculus, " $d$ " means "a

\footnotetext{
${ }^{4}$ An example of this could be: given a definite integral $\int_{a}^{b} f(x) d x$, one could think of the length of $d x$ as $10^{-50}$. This is a finite value, but immeasurable with current instrumentation (for reference, the smallest unit of time ever measured is $10^{-21}$ seconds). This $d x$ would thus have a value that is a real number, yet in the physical world, such a number is immeasurable, and in a sense, infinitesimal.
} 
little bit of," (Thompson, 1910, p. 1), later designating the $d x$ in the construction of the derivative as "indefinitely small" (Thompson, 1910, p. 12), and describing the $d x$ in the construction of an integral as "infinitesimally small" (Thompson, 1910, p. 186). Still, even if his $d x$ cannot be quantified as explicitly as the other differentials in the books summarized in this section, the idea of the differential as the primary building block of first-year calculus is novel and worth mentioning.

Differentials as algorithmic tools. Dray and Manogue (2010), while not advocating for formal nonstandard analysis, nevertheless champion a differential-based calculus in which differentials are used simply as algorithmic tools. In this version of calculus, a solution process to calculus problems can begin with taking either differentials of functions or what they call differentials of equations. For example, given the equation $a^{2}+b^{2}=c^{2}$, one could find the differential form of this equation $2 a d a+2 b d b=2 c d c$. This differential form has many uses: it could be used as a part of the solution to an optimization problem, or, if one divides by $d t$, $2 a \frac{d a}{d t}+2 b \frac{d b}{d t}=2 c \frac{d c}{d t}$, one gets an equation that could be used to help solve a related-rates problem. In cases like these, no thought is given to any quality of the differentials (Do they come from limits? Do they have a size?), but the differentials are important algorithmic tools.

\section{Differential Conceptualizations in Randomly-Selected Textbooks}

Before conducting my first, exploratory, study (Chapter 4), I wished to create a frame of reference by looking at how (or if) differentials were conceptualized in a selection of randomlyselected textbooks. I conducted two such surveys, the first involving twelve books (Barnett \& Ziegle, 1989; Breusch \& Ogilvy, 1969; Ellis \& Gulick, 1988; Fisher \& Ziebur, 1965; Gleason, Hughes-Hallett, \& McCallum, 2013; Hughes-Hallet, et. al., 2006; Larson \& Edwards, 2014; Mizrahi \& Sullivan, 1982; Rees \& Sparks, 1969; Stein, 1967; Stewart, 1987; Stewart, 2015) 
containing material found in traditional first- and second-semester calculus courses, and the second involving three books (Boyce \& DiPrima, 2012; Stewart, 1987; Zill, 1997) containing information on basic ordinary differential equations.

For the first survey, I analyzed and compared how (or even if) differentials were described within the sections of the textbooks that introduced or defined definite integrals, indefinite integrals, differentials of functions, and integration by substitution. The results I found were fairly uniform: with some exceptions, all of the books seemed to utilize the same particular conceptualizations. Specifically ${ }^{5}$ :

- The only area in which the books showed a significant split in opinion was in whether the symbol “ $\frac{d y}{d x}$ ” initially represented a fraction made up of two quantities. This notation is normally first mentioned after the definition of derivative is developed through the use of limits applied to a difference quotient. The symbol $\frac{d y}{d x}$ is then noted merely as an alternative to the "prime" notation $f^{\prime}(x)$ for derivatives, and, indeed, Stein (1967) noted that "at this point, we should no more interpret the symbol $\frac{d y}{d x}$ as a quotient than the symbol 8 as two zeros. The only books that definitively said at first that $\frac{d y}{d x}$ could be considered a quotient were Barnett and Ziegle (1989), Breusch and Ogilvy (1969), Fisher and Ziebur (1965), and Mizrahi and Sullivan (1982). However, after the introduction of the differential of a function, all of the books gave some variation of the idea that one could think of $\frac{d y}{d x}$ as a quotient of particular values.

\footnotetext{
5 There was more than one order in which the textbooks presented these subjects. I choose to discuss them in the following order in order to align them with the dissertation's interview protocol.
} 
- All but one of the books stated that the $d x$ in a definite integral was merely notation with its only purpose to indicate the variable of integration. Only Gleason et al. (2003) and Hughes-Hallet et al. (2006) mentioned that the $d x$ in the definite integral comes from the $\Delta x$ found in a Riemann sum. Interestingly, Stewart (2015) stated that this particular Leibniz notation was chosen in order to denote which variable was involved in the limit process, which seems incorrect, as Leibniz notation preceded epsilon-delta limit notation by a couple of centuries.

- Only Larson and Edwards (2014) described the $d x$ in an indefinite integral as indicating the variable of integration. None of the other books discussed any role of the $d x$ in an indefinite integral at all, stating versions of the idea that the indefinite integral symbol $\int f(x) d x$ was merely a representation for "antidifferentiation of $f(x)$."

- The nine earliest-published books (Barnett \& Ziegle, 1989; Breusch \& Ogilvy, 1969; Ellis \& Gulick, 1988; Fisher \& Ziebur, 1965; Hughes-Hallet, et. al., 2006; Mizrahi \& Sullivan, 1982; Rees \& Sparks, 1969; Stein, 1967; Stewart, 1987) that mentioned the differential of a function introduced it in the context of linear approximation of nonlinear functions. They gave the formula $d y=y^{\prime}(x) d x$, with all but Fisher and Ziebur (1965) stipulating that the $d x$ is equal to $\Delta x$, and can be any real number. Fisher and Ziebur (1965) instead stated that one can treat the relationship $d u=u^{\prime}(x) d x$ as if $d u$ and $d x$ were numbers, but that they were not going to formally interpret them as numbers. The three most-recent books (Gleason, Hughes-Hallett, \& McCallum, 2013; Larson \& Edwards, 2014; Stewart, 2015) introduced differentials of functions without the same differential notation, with Larson \& Edwards and Stewart presenting the idea of linearization with the notation $f(x)-f(a) \approx$ 
$f^{\prime}(a)(x-a)$ and introducing the previous notation afterward, while Gleason, et al. (2013) only used this newer notation.

- Every book approached the evaluation of an integral that required substitution by the usual method of determining a proper $u(x)$ and using the relation $d u=u^{\prime}(x) d x$ to facilitate substitution. However, with two exceptions, there was no discussion as to the nature or roles of the differentials used throughout this process. The two books that were exceptions discussed the notation in more detail, specifically mentioning that we can think of these notations as differentials (Stewart, 2015) or that the re-written notation $d u=\frac{d u}{d x} d x$ may look like cancellation but is not (Gleason, et al., 2013).

For the second survey, I compared and contrasted how (or if) differentials were described within the sections that introduced separable and exact differential equations. For the most part, differentials were not discussed at all and merely used as algorithmic tools in the various solution methods. The only exception was when Zill (1997) used the idea that a " $d$ " can be a cue for the act of differentiation (specifically when $d(x y)=y d x+x d y$ was given as a part of a solution method)

Looking at these particular books, one can conclude that there seemed to be a vague, general agreement as to the nature of differentials in each contexts, but that this nature varied drastically from context to context. To describe them using similar terms as the descriptions in the literature review, the books seemed to proceed through the following conceptualizations: differentials as merely notation or differentials as "small" (derivative notation), the differential indicates a variable (definite integration), differentials are merely notation (indefinite integration), differentials are real numbers used for approximation (when defining "differential of a function" 
and linear approximation), and the differential is an algorithmic tool (integration by substitution and solving ordinary differential equations.)

\section{Summary of the Literature Review}

The list of conceptualizations that I give in this section is somewhat large for only one mathematical symbol. Indeed, I wonder if there are any other mathematical symbols that invite so many different interpretations. To add to the chaotic nature of the multiple conceptualizations of this symbol, nothing within the list of conceptualizations is meant to suggest that one can only hold one of these conceptualizations at a time. Dray and Manogue (2010) mention that they tell their students that differentials can be viewed as arbitrary changes in given quantities, a shorthand notation for limits, and hyperreal infinitesimals. Each of these are called "reasonable definitions" (p. 96), suggesting that there is nothing incorrect about holding multiple views of the $d x$, as mentioned in the second half of Tall's quote ${ }^{6}$. A theoretical perspective that allows for the possibility of accepting multiple conceptualizations will be addressed in the next section.

\footnotetext{
6 "Giving a modern meaning to these terms that allows a consistent meaningful interpretation for all contexts in the calculus is possible but not universally recognized. On the other hand, failing to give a satisfactory coherent meaning leads to cognitive conflict which is usually resolved by keeping the various meanings of the differential in separate compartments $\left(\frac{d y}{d x}=\lim _{\Delta x \rightarrow 0} \frac{\Delta y}{\Delta x}\right.$ in differentiation, and $d x$ means "with respect to $x$ " in integration)." (Tall, 1993, p. 6)
} 


\section{THEORETICAL PERSPECTIVE}

The introductory section of this dissertation showed some of the mathematical contexts in which differentials appear and the literature review listed some conceptualizations one could have of these differentials in these contexts. However, nothing in the previous sections of this dissertation are meant to suggest that one must choose only one conceptualization for the differential. The quote from David Tall (1993) presented in this dissertation's introduction suggests that it is possible that individuals might use different conceptualizations for the differential depending upon the context in which they are found. Because it is possible for individuals to have many and varied meanings for the differential, Tall and Vinner's concept image (1981) is an appropriate theoretical perspective for this research.

Tall and Vinner (1981) define concept image as "the total cognitive structure that is associated with the concept, which includes all the mental pictures and associated properties and processes" (p.152). I believe that the words "total" and "all" in that quote are important. As mathematicians, we can draw upon many formal definitions, theorems, and examples while thinking about a mathematical topic, but our concept images do not have to be restricted to only these formal elements. Sometimes, mathematical concepts may be used informally at first, and the processes used by individuals to help them understand these initial informal uses might stay with the individual even after a formal treatment of these concepts are introduced. To give a personal example, my concept image of infinitesimals does contain formal definitions and theorems, but it also contains intuitive, non-formal mental images like Keisler's (2012) idea that the solution to the differential equation $\frac{d y}{d t}=f(t, y)$ can be thought of as "a moving point controlled by an infinitesimal driver," who travels point by point, making sure his "steering wheel" is turned to the correct slope at every point (Figure 2). This presentation is quite informal, yet it is a mental picture 


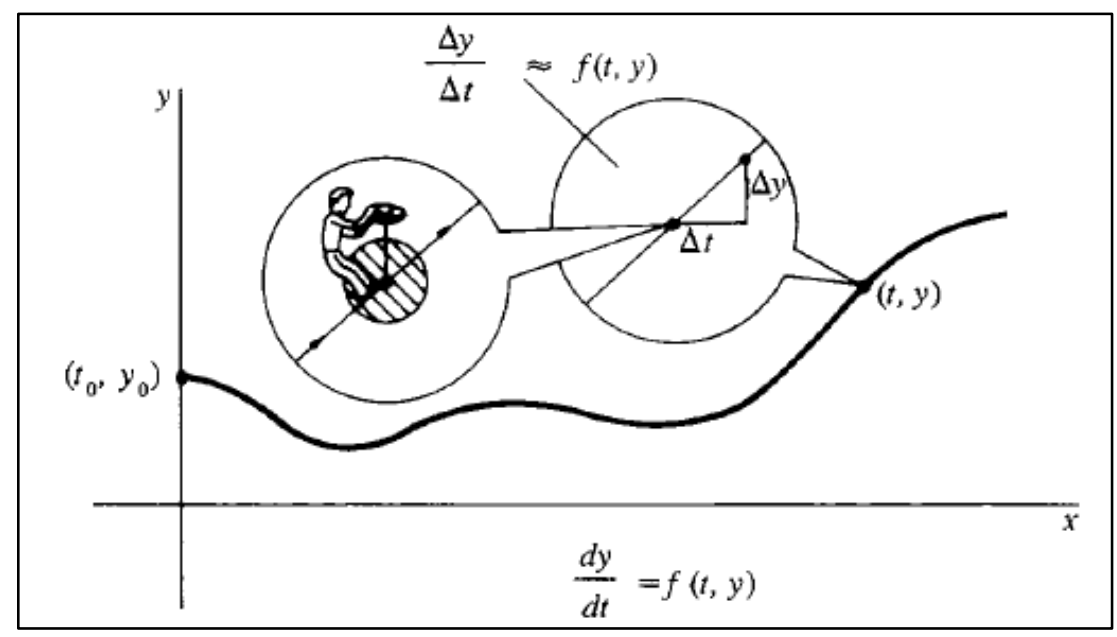

Figure 2: Keisler's "infinitesimal driver" (Keisler, 2012, p.848).

that still "appears" in my thoughts alongside formal solution methods and theorems when thinking about a first-order ordinary differential equation. It is possible that certain experts might have similar informal ideas in their overall concept images of differentials.

Thus, the range of ideas contained within one's concept image of a topic can be extensive, and within this extensive range, there might be parts of a conflict image that seem to contradict other parts. These conflicting parts are called potential conflict factors, unless they are evoked at the same time, in which they create conflict and become cognitive conflict factors (Tall \& Vinner, 1981). A hypothetical example of a potential cognitive conflict factor involving differentials could come from an individual whose concept image of the symbol " $\frac{d y}{d x}$ " includes the idea that it should not be thought of as a fraction (and thus the $d y$ and $d x$ cannot be separated), but whose concept image of separable differential equations includes the idea that one must separate the $d y$ and $d x$ in the symbol $\frac{d y}{d x}$ in order to solve the separable equation. It is possible that these conflicting ideas would remain as only a potential conflict factor until such time as this particular individual were presented with both statements simultaneously: "I notice that you separated the $d y$ and $d x$ when 
solving this equation, but you said earlier that $\frac{d y}{d x}$ did not represent a ratio." Perhaps the individual in this example would have no problem accepting both statements; but if the simultaneous presentation of both statements created conflict, then he or she would have a cognitive conflict factor which would need to be resolved.

Tall's quote from the introduction of this dissertation suggests that one might draw upon different parts of one's concept image at different times. The part of the concept image which is in use at a particular time is called the evoked concept image (Tall \& Vinner, 1981). To use the previous example, the aforementioned mathematician might have a robust concept image of the differential in which it is both a piece of the non-ratio notation " $\frac{d y}{d x}$," and also an algebraic entity that can be manipulated when solving a differential equation. Each of these views can have images, definitions, informal ideas, examples, counterexamples, and other mathematical phenomena attached to them. When presented with an expression that includes " $\frac{d y}{d x}$ ", the context of the presentation could cause one of these views, as well as some of the attached phenomena, to become prominent in this mathematician's mind. The totality of what becomes prominent would be the evoked concept image for that particular differential.

Individuals may have a personal concept definition, or a collection of words used to describe a particular concept (Tall \& Vinner, 1981). An individual's personal concept image and personal concept definition are not meant to be static entities; indeed, as he or she learns more about the concept and thinks about it in greater detail, one would hope that he or she refines and/or expands his or her concept image and definition. At some point, perhaps one's personal concept definition will approach what Tall and Vinner (1981) call the formal concept definition, which is the collection of words used to describe the concept that is accepted by the general mathematical community. 
On the surface, it may appear that there is no formal concept definition for the differential, since some of the conceptualizations listed in the Literature Review are not at all similar (the idea of a $d x$ as a concrete, small amount or change versus the idea of $d x$ as a variable indicator, for example.) However, the above literature mostly describes student conceptualizations of only one particular differential, while my dissertation involves interviewing experts about their conceptualizations of multiple differentials. Perhaps these experts, who have studied mathematics longer and in more detail than students, will have more finely-tuned responses that converge to a formal concept definition. If not, then perhaps strong personal concept definitions will emerge, or instances of cognitive conflict will occur. 


\section{PREVIOUS WORK}

Before beginning the work needed for this dissertation, I conducted two smaller-scale studies. The main goal of these studies was to provide forums in which I could both practice and improve my ability to conduct a productive interview as well as help test and refine possible interview questions to be used in my dissertation work. The results of these smaller-scale studies are explained in more detail in McCarty and Sealey (2017) and McCarty and Sealey (2018), and I review some of them below.

\section{Exploratory Study}

The definitions of and explanations for differentials given in the textbooks I surveyed were not uniform and usually not detailed. This seemed to show that no formal concept definition of the $d x$ exists. The realization of this led me to wonder how uniform or detailed the concept images of various mathematicians might be. I designed an interview protocol to be given to experienced mathematics faculty in an attempt to understand their personal concept images. I wished to determine if these concept images would be more consistent and well-formed than the ideas presented in the textbooks.

\section{Methods}

The entire interview protocol is given in Appendix A, but a summary of the mathematical contexts that were discussed is shown in Table 1. Four professors of mathematics from a large research university responded to emails requesting interviews. Participants Sonya, Johnny, and Jackson (pseudonyms) each had research and/or teaching experience in analysis and differential equations, while Kurtis' research areas included combinatorics and graph theory. Because of the complexity of some of their answers and my wish to explore their answers further, Sonya and Johnny agreed to follow-up interviews. All of the interviews except Johnny's were videotaped, 


\begin{tabular}{|c|c|}
\hline $\begin{array}{l}\text { Table } 1 \\
\text { Exploratory Study's Protoc }\end{array}$ & Expressions \\
\hline Categories & Symbols, Definitions, or Situations Containing $d x$ \\
\hline Integrals & $\int f(x) d x, \int_{a}^{b} f(x) d x$, and $\int_{b}^{a} f(x) d x$ \\
\hline Definitions and Notation & $\begin{array}{l}\text { If } y=y(x) \text {, the notation } \frac{\frac{d y}{\frac{d x}{d x}} \text { and definition } d y=y^{\prime}(x) d x}{d x} \\
\text { If } x=x(t) \text {, the notation } \frac{\frac{d x}{d t}}{d x} \text { and definition } d x=x^{\prime}(t) d t\end{array}$ \\
\hline Integration by Substitution & $\begin{array}{c}\int_{1}^{4} \frac{\cos \sqrt{x}}{2 \sqrt{x}} d x \text { versus } \int_{1}^{2} \cos x d x \\
\text { after } \int_{1}^{4} \frac{\cos \sqrt{t}}{2 \sqrt{t}} d t \text { used the substitution } d x=\frac{1}{2 \sqrt{t}} d t\end{array}$ \\
\hline Two ODEs & $\begin{array}{l}\text { 1) The separable equation } \frac{d y}{d x}=g(y) h(x) \text {, and the solution } \\
\text { steps } \frac{1}{g(y)} d y=h(x) d x \text { and } \int \frac{1}{g(y)} d y=\int h(x) d x \\
\text { 2) The exact equation }\left(2 x y-9 x^{2}\right) d x+\left(x^{2}+2 y+1\right) d y=0\end{array}$ \\
\hline
\end{tabular}

Johnny's exclusion per his request.

At this point in my research, I had not decided on a particular methods of data analysis, so analysis of the data I collected in this study was informal. The videotaped interviews were first fully transcribed, and each transcript was read all the way through in order to see if I could gauge the level of consistency (if any) throughout all of the subjects' answers. I then devoted more attention to the portions of the interviews that addressed the four mathematical contexts containing differentials. Specifically, I looked for and noted words or phrases that seemed to indicate what "quality" the subject thought differentials held: for example, were differentials merely notation, encapsulated mathematical entities, or somewhere in between? I then used these "quality" words and phrases to refine my first, rough ideas of the subjects' concept images that I obtained from the first read-through. Finally, I attempted to distill them into smaller, more focused ideas. Since 
Johnny requested that his interviews not be videotaped, during his first interview, I endeavored to take careful notes of the "quality" of differentials and immediately upon the interview's conclusion, wrote out my thoughts as to how I perceived his overall concept image. During his second interview, I asked follow-up questions to ensure that my notes and impressions were accurate.

\section{Data}

Preliminary analysis indeed showed that while all four interview subjects were fairly consistent within their responses throughout the different contexts, their responses differed from each other's in many ways, with no two subjects agreeing with one another throughout the entire protocol. Thus, a single, formal concept definition did not manifest. Summaries of the subjects' responses for each of our four contexts and my interpretation of some of the subjects' personal concept images follow:

The $\boldsymbol{d} \boldsymbol{x}$ in definite and indefinite integration. Sonya and Jackson stated that the $d x$ in a definite integral comes from a limiting process applied to the width represented by the bases of Riemann Sum rectangles. Johnny initially described the $d x$ similarly, as arising from the limit of "cuts in the interval between $a$ and $b$ on the $x$-axis," but later said it was only "a conventional symbol borrowed from differentiation." Kurtis defined both the $d x$ in definite and indefinite integrals as part of notations that, respectively, represented the limit of a Riemann sum and a family of functions. He later mentioned that the $d x$ comes from the $\Delta x$ in a Riemann sum, but only as a matter of notation and not because $d x$ is a concrete entity all to itself. All subjects generally claimed that they viewed the $d x$ in an indefinite integral no differently than they viewed the $d x$ in a definite integral, whether that $d x$ was viewed as a mathematical entity or as merely notation. 
The $\boldsymbol{d} \boldsymbol{x}$ in definitions and Leibniz notation. Kurtis said that $d y=y^{\prime}(x) d x$ if and only if $\frac{d y}{d x}=y^{\prime}(x)$, but did not feel that this meant that one could simply multiply or divide by $d x$ to go from one form to the other. Johnny and Jackson had no caveats nor conditions; multiplying or dividing by $d x$ to go from one form to the other was always acceptable to them. Sonya agreed that such multiplication or division was not possible if we view the symbol $\frac{d y}{d x}$ as a derivative, and she was the only subject who seemed to imply that there was a structural difference to her in the two relationships $d y=y^{\prime}(x) d x$ and $\frac{d y}{d x}=y^{\prime}(x)$ :

Sonya: $\quad$ But because I see it a lot of times where with students, it's not like that $\left[\right.$ indicates $\left.\frac{d u}{d x}=\frac{1}{2 \sqrt{x}}\right]$ is a fraction and you just move around the $d x$ and rewrite it like that [indicates $\left.d u=\frac{d x}{2 \sqrt{x}}\right]$.

Interviewer: That's not what's happening here?

Sonya: $\quad$ No, that's not the way I see it. That $\left[\right.$ indicates $\left.\frac{d u}{d x}\right]$ is derivative of $u$ with respect to $x$, and that $\left[\right.$ indicates $\left.d u=\frac{d x}{2 \sqrt{x}}\right]$ is more small changes of $u$ and $x$ - more like a differential thing.

The $\boldsymbol{d} \boldsymbol{x}$ in integration by substitution. For reference, the initial integral was $\int_{1}^{4} \frac{\cos \sqrt{t}}{2 \sqrt{t}} d t$ and during its evaluation, all subjects used the substitution $x=\sqrt{t}$ and $d x=\frac{1}{2 \sqrt{t}} d t$. All subjects except Sonya seemed to feel that no matter how one viewed the $d x$ in " $d x=\frac{1}{2 \sqrt{t}} d t$ ", once the substitution into the initial integral was made, the $d x$ in the new integral $\int_{1}^{2} \cos x d x$ was only an "indicator" signifying the new variable of integration. Sonya, however, kept a view that the $d x$ in " $d x=\frac{1}{2 \sqrt{t}} d t$ " retained some additional meaning. She believed that the $d x$ and $d t$ in the above 
relationship were entities that resided on different levels; one could say that $d x=x^{\prime}(t) d t$ was on the level of $t$, but if $t$ were a function of $s$, so that $d t=t^{\prime}(s) d s$, then one would be at the level of $s$. No matter what level, though, the differentials were entities and "they are all going to zero," although some more quickly than others. This idea that these two versions of $d x$ have different sizes was also expressed by Jackson. He mentioned that while the initial dummy variable (in $\int_{1}^{4} \frac{\cos \sqrt{x}}{2 \sqrt{x}} d x$ ) and the transformed dummy variable (in $\int_{1}^{2} \cos x d x$ ) had similar roles as infinitesimal widths, we could still think of them as different, since one was the limit as $n$ goes to infinity of $\frac{4-1}{n}$ while the other was the limit as $n$ goes to infinity of $\frac{2-1}{n}$ : "Even though they're both infinitesimal, they're still not the same."

The $\boldsymbol{d} \boldsymbol{x}$ in separable and exact ODE's. Johnny and Jackson, as before, had no problem with multiplying or dividing by $d x$ in order to solve a differential equation. Sonya felt that even though it may appear that one could multiply by $d x$ in order to separate variables in the separable equation, what is really happening instead is that one multiplies by $\Delta x$ and then passes through a limit to turn $\Delta x$ into $d x$. Kurtis agreed with the idea that we are not really multiplying by $d x$, but seemed to think that it was always fine to proceed as if that is what were really happening. Similar responses occurred during the explanations of the exact ODE. Sonya was still uncomfortable with the idea of "moving the $d x$ around" but admitted that it is how solving differential equations is usually taught. Johnny and Jackson did not have this discomfort, and Kurtis declined to answer, stating that he was not as familiar with exact differential equations.

\section{Results}

The amount of disagreement in preceding paragraphs shows that there was no overall concept definition for the differential within my four interview subjects. However, the interviews of Johnny, Kurtis, and Sonya were at least fairly consistent from start to finish, containing similar 
views of differentials across multiple contexts. I will summarize their central themes in the remainder of this section.

Johnny repeatedly stated that differentials were "meaningful only in their relation to one another," meaning that the number of differentials in a mathematical situation determined the character of the differentials. If a mathematical situation had two related differentials present, then those differentials were concrete entities that could be manipulated, while if only one differential were present, then that differential was merely notation. For example, in a separable differential equation $\frac{d y}{d x}=f(x) g(y)$, one is allowed to multiply or divide by a $d x$ since we have "a relation of differentials [ $d y$ and $d x]$," but in the definite integral $\int_{a}^{b} f(x) d x$, the $d x$ is merely a notation that indicates the variable of integration, since there is only one differential present in the expression.

Kurtis said at least one time per context that all of the manipulations done with differentials were merely products of a "perfectly good notation." The efficacy of differential notation makes it easier for educators to introduce concepts like the Chain Rule or integration by substitution, but within such mechanizations, the actual differentials have no substance nor meaning. Thus, while it may appear that differentials might be multiplied, divided, and/or cancelled, Kurtis was adamant that these operations were not really what was happening, and that the differential notation was simply a shorthand for the more advanced mathematics required to understand these concepts: “Again, it's good notation, so it's [multiplying by $d x$ ] not what's really happening, but that's what you can do and get the right solution. So one more bean for 'this notation is good'."

A central image of Sonya's concept image was that the $d x$ did not have any meaning on its own if it was a part of the symbol $\frac{d y}{d x}$, which represented a derivative. She noted the convenience 
of simply saying "multiplying by $d x$ " when given the notation $\frac{d y}{d x}$ and that it was helpful for instruction, but that we should be more careful about telling our students "we can multiply by $d x$ " in these situations. Instead, one should understand that the idea of "multiplying by $d x$ " is a shorthand for "multiplying by a $\Delta x$ and then passing through a limit."

\section{Pilot Study}

The results of this exploratory study were encouraging. It is one thing to see many and varied conceptualizations in literature that addresses student interpretations; it is another thing to see many and varied conceptualizations espoused by expert mathematicians. Thus, I wished to continue research, and I felt that this first study could be extended in two ways. First, since physicists also use differentials in their work and since I used Physics Education Research in my literature review, I wanted to explore and compare the concept images of both mathematicians and physicists. Second, I wanted to ask experts how they would like their students to conceptualize these differentials. This idea arose from Kurtis often discussing how his students seem to view the ideas behind the notation. His remarks suggested to me that instructors could not only accept but also approve if their students held concept images that differed from their own, and I wished to explore this. I aimed to address these two ideas in a pilot study that was conducted during the next year.

\section{Methods}

The protocol for this study is given in Appendix B and outlined in Table 2 below, but the two most notable changes from the exploratory study's protocol are summarized here. First, the situations containing differentials were streamlined into what I believe to be better sections: 1) definite and indefinite integration, $\int_{a}^{b} f(x) d x$ and $\left.\int f(x) d x, 2\right)$ the symbol $\frac{d y}{d x}$, and 3$)$ the relationship $d y=y^{\prime}(x) d x$. Each section began by showing and discussing the mathematical 


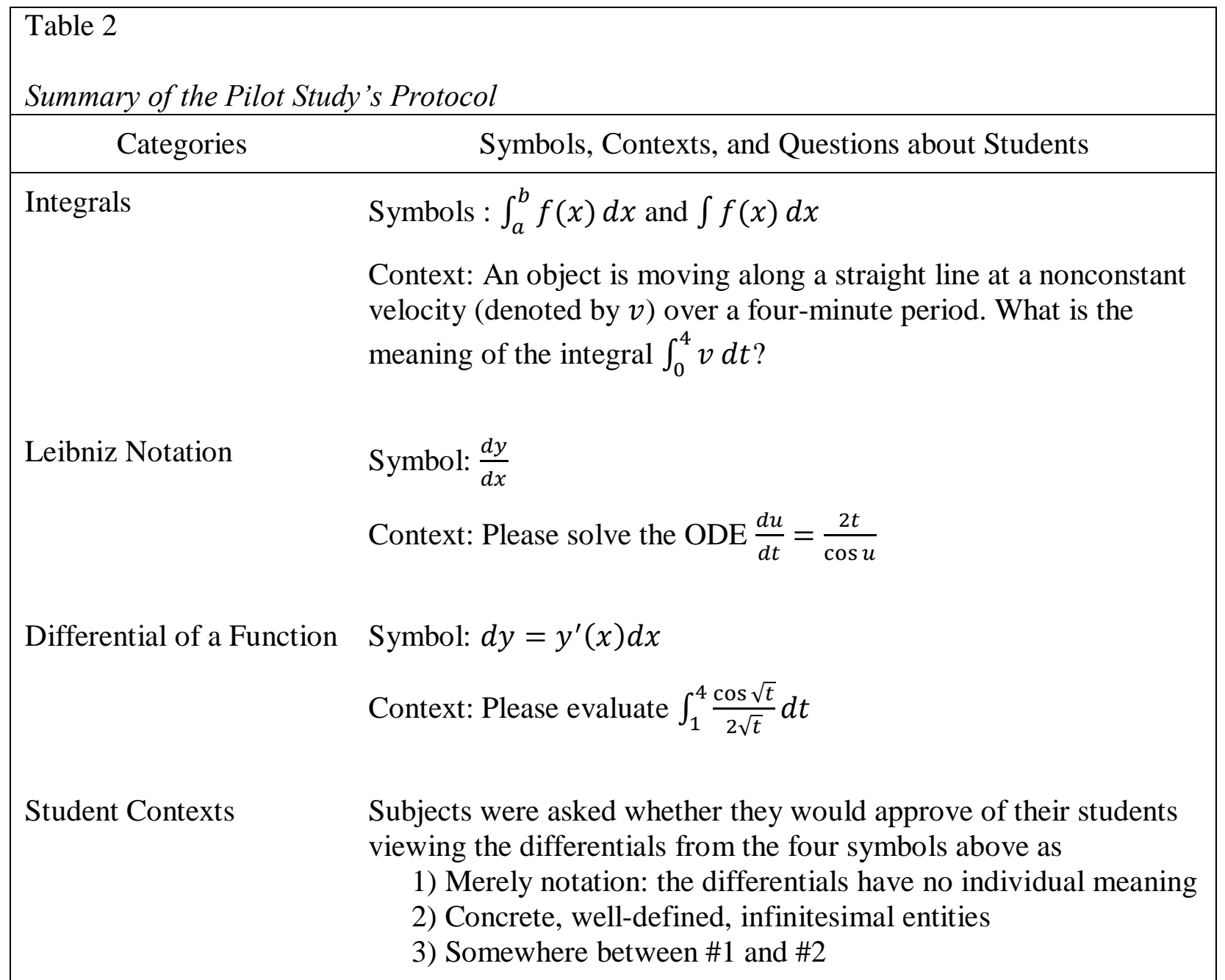

symbol and ended with a situation in which the symbol was used within some context. Second, questions were included to gauge both how the subject felt about conceptualizations of differentials that were different from his or her own, and the differences, if any, between the concept image that the instructor held and the concept image that he or she wished for his or her students to hold.

Two mathematicians and one physicist with teaching experience were interviewed, all of whom were currently teaching at the same large research university. Mathematicians Tanya and Li Mei had various levels of experience teaching first-, second-, and third-semester calculus. Physicist Darrius, who has an undergraduate degree in both mathematics and physics and a Masters and Ph.D. in physics, had taught plasma and calculus-based electricity and magnetism courses to 
students of various levels. A second physicist was interviewed mostly to test if an interview could be conducted over Skype and recorded with OBS Studio. The interview was successful and thus, I determined that internet interviews were a viable option for my research. Because this second physicist was not asked to sign a consent form, her responses are not included in this paper.

As in the exploratory study, no formal method of data analysis was used. Interviews were videorecorded and then transcribed. Within these transcriptions, I first searched for answers that were unexpected or seemed unique in some way, then used these unique answers as mainstays upon which I attempted to build an interpretation of the subject's overall concept image. I then compared and contrasted these interpretations, not only to find areas of agreement and disagreement, but also to see whether the subjects' specific answers to certain questions suggested ways in which the current protocol might be improved.

\section{Data and Results}

As with the exploratory study, each subject's responses differed from one another in many places, confirming, again, that a single, formal concept definition for differentials did not exist. Summaries of the subjects' responses follow.

The $d x$ in definite and indefinite integration. It is within integration where I first noticed a split between the views of the mathematicians and physicist participating in my study. Tanya and Li Mei both initially stated that the role of the $d x$ was to denote the variable of integration (both referenced multivariable calculus and noted the possibility that the integrand might contain more than one independent variable) and later stated that the $d x$ in a definite integral was a representation of a Riemann sum rectangle and invoked the idea of limit. In contrast, Darrius initially stated that the $d x$ in both the definite and indefinite integrals were infinitesimal segments 
in $x$. He did mention the idea of taking a finite amount and shrinking it to zero, thus also implying a limit process, but his primary idea seemed to be $d x$ as an infinitesimal amount.

Differentials in Leibniz derivative notation. While the integration section suggested a mathematician/physicist split between the individuals in this specific study, it was this section that showed this split was not absolute. Tanya initially said that $\frac{d y}{d x}$ was merely notation and not a ratio of $d y$ and $d x$ and that the role of the $d y$ and $d x$ was to identify the dependent and independent variables. Li Mei and Darrius said that $\frac{d y}{d x}$ could be thought of as both one notation or as a ratio between two entities, depending upon the context in which the symbol was presented. For example, thinking of $\frac{d u}{d t}$ as a ratio was necessary when solving the differential equation $\frac{d u}{d t}=\frac{2 t}{\cos u}$, as one should "multiply by $d t$ " to facilitate the necessary separation of variables. Tanya also agreed that it was fine to say "multiplying by $d t$ " when separating variables, but seemed at first to view this separation as an algorithmic step, rather than as a validation that $\frac{d u}{d t}$ was a ratio of two quantities.

Differentials in the relationship $d y=y^{\prime}(x) d x$. This question had the largest variety in answers. Upon seeing this relationship, Darrius wanted to bring the $d x$ over to get $\frac{d y}{d x}=y^{\prime}(x)$, while saying that $y^{\prime}(x)$ is equal to the ratio of how $y$ changes, given a small change in $x$. Li Mei primarily viewed the initial relationship as a means to approximate $d y$ given a measurable $d x$. Tanya said that she did not know how to interpret this relationship because she claimed to have never seen a relationship like this before. When asked to integrate $\int_{1}^{4} \frac{\cos \sqrt{t}}{2 \sqrt{t}} d t$, which requires the substitutions $u=\sqrt{t}$ and $d u=\frac{1}{2 \sqrt{t}} d t$, Tanya opined that the $d u$ and $d t$ were only symbols that were used to represent what the variable of differentiation was on both sides. Li Mei and Darrius 
both said that $d u=\frac{1}{2 \sqrt{t}} d t$ tells us exactly how the rate of change of $u$ differs from the rate of change in $t$.

Deltas and infinitesimals. Tanya seemed to go back and forth on her opinion whether $d x$ and $\Delta x$ had the same meaning. Early statements showed that, to her, $d x$ is a variable indicator while $\Delta x$ is a small interval: "So the $d x \ldots$ this is here to determine that we are taking the integral respect to variable $x$ " and "...by Riemann sum, we can say that, OK, we have a small interval $a$ to $b$, which is an interval in $x$-axis ... because of the $\Delta x$, a small portion of this interval." However, later statements seemed to suggest that one can think of the integral's $d x$ as a $\Delta x$ : "Now we can interpret this $d x$ as $\Delta x$ " and "so we have $f(x)$ times $\Delta x \ldots$ if you go back to this notation $\left[\int_{a}^{b} f(x) d x\right]$ we have $f(x)$ times $d x$, this represents the same $\Delta x$." Li Mei kept the two ideas distinct, stating that $\Delta x$ had a finite measure, and that $d x$ represented making the $\Delta x$ smaller until it "almost disappears." Thus, to her, $d x$ was small and nonzero, but she said that she didn't know and never really thought about if it had a measure like she believes $\Delta x$ does. Darrius never mentioned "Deltas" in any of his answers, and thus, they weren't addressed until the end of the interview when we were discussing possible student conceptualizations, some of which included Deltas. When asked if he ever thought about Deltas, Darrius said that any time in the interview when he said that $d x$ was "taking a finite distance and then letting that distance go to zero," he considered that initial finite distance as $\Delta x$. This perhaps shows that, even though he never used the word "Delta" during his interview, he does conceptualize the $d x$ as a small $\Delta x$.

All subjects were asked to comment on the following statement ${ }^{7}$, which was included as a possible means to help gauge how the subjects conceptualized the "size" (if any) of the $d x$ : It is

\footnotetext{
${ }^{7}$ This question was meant to reference Courant \& John's (1965, p.184) "physical infinitesimals" (Page 15 of this dissertation)
} 
OK to conceptualize differentials such as the $d x$ in $\int_{a}^{b} f(x) d x$ or $\frac{d y}{d x}$ as nonzero, finite quantities, small enough to fall under any measurable scale. (For reference: Diameter of electron: less than $10^{-16} \mathrm{~cm} /$ Smallest unit of time ever measured: $10^{-21} \mathrm{sec}$ )" Tanya did not like this idea, since even numbers less than $10^{-21}$ were still finite, and, to her, finite numbers were "not enough" to represent the size of $d x$. Li Mei and Darrius were fine with the general statement, but did not want to assign a specific number to $d x$, since once that number was assigned, one could not go any smaller.

\section{Instructor concept images versus the images they would accept from their students.} A summary of how the subjects' views compare with the views they would accept their students having is given in Table 3. Similar to how no experts agreed on every aspect of their concept image, the lists of conceptualizations that the experts would approve were also different. Table 3 is only a rough representation of the subjects' views for two reasons: it is difficult to distill long interview responses into only a checkmark or an 'X," and the questions about their students' views were only a few questions given at the end of the interview. I believe that more intense and prominent questioning in future interviews would elicit more information.

Notable differences between the subjects' conceptualizations and the conceptualizations they would accept from their students are summarized below:

- $\int_{a}^{b} f(x) d x$ : Li Mei said that it was fine for her students to view this $d x$ as an infinitesimal amount, even though she never stated that she personally viewed this $d x$ in this way. To her, viewing this $d x$ might be useful for student understanding: “ $\ldots$ if they think of having lots and lots of values for $f$, and lots and lots of tiny values for $d x$, and then that this [points at the integral sign] actually means summation - if this helps them to understand the stuff, that's good." Darrius said that he would accept a student linking this $d x$ to a 


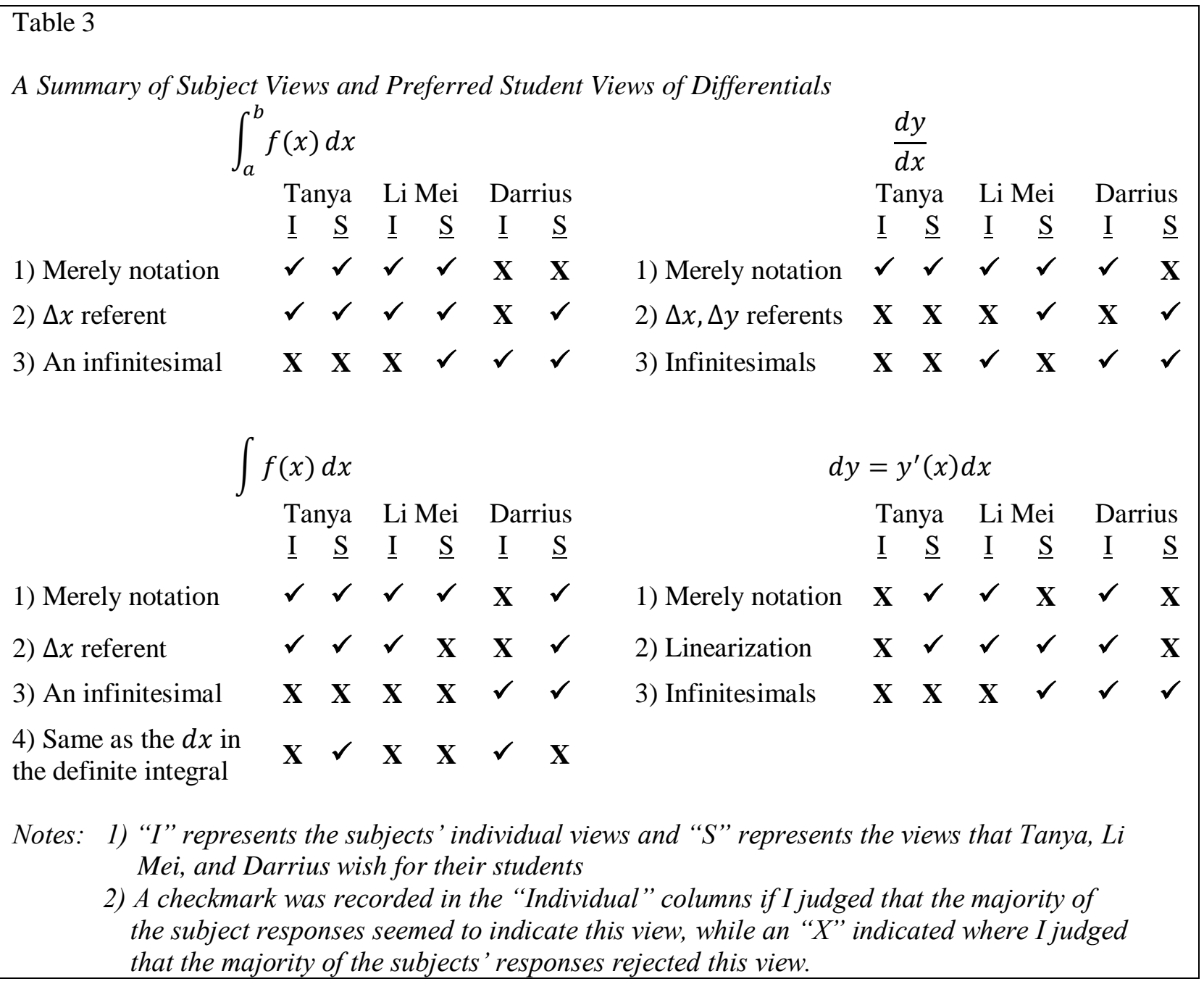

Riemann sum's $\Delta x$, but claimed that this idea would never be stated in the physics world or by a physics student.

- $\frac{d y}{d x}$ : Tanya only wanted her students to think of this as a notation that stands for "the derivative of $y$ with respect to $x$." Li Mei was fine with students holding this view, as well as the view that $\frac{d y}{d x}$ arises from the limit of $\frac{\Delta y}{\Delta x}$, even though she did not personally subscribe to that view during her interview. Similarly, Darrius accepted the "limit" view even though he did not state it in his interview. 
- $\quad d y=y^{\prime}(x) d x$ : Both Li Mei and Darrius felt that the two ideas of "in a small neighborhood around $x$, the tangent line is an approximation of the curve $y(x)$ " and " $d y$ is an infinitesimal that is proportional to an infinitesimal $d x$ " were both acceptable. They did not want their students to think that $d y=y^{\prime}(x) d x$ was only a notation that we used when needed, like in integration by substitution, as they felt that the $d y$ and $d x$ should have some meaning. Tanya, despite earlier claiming that she had not seen this notation before, nevertheless thought that the above "neighborhood" idea, as well as the idea that using $d y=y^{\prime}(x) d x$ without meaning were both fine.

\section{Summary of the Previous Work}

Both of these initial studies clearly showed that not only was there was no formal concept image of the differential, but also that there was significant variation in the experts' personal concept images. While the second study's inclusion of a physicist and preferred student views suggested that these were valid areas of exploration, I believe that these areas are best suited for future work. Thus, my dissertation focuses only on the variety and consistency of the views possessed by expert mathematicians. 


\section{METHODS}

My research questions were chosen to emphasize the focus on mathematicians' views of the differential, and I repeat them here for reference:

1. How extensive are the concept images of differentials held by expert mathematicians?

2. What levels of consistency, if any, exist in the concept images of the differential within each individual?

3. What levels of consistency, if any, exist in the concept images of the differential among all mathematicians interviewed?

The remaining information in this section describes the methods of data collection and data analysis undertaken for this dissertation.

\section{Data Collection}

There were two groups of interviews conducted. For the first group of interviews, I interviewed seven individuals who taught at the same large research university. For the second group of interviews, one (Xavier) was a mathematician who taught at the same university as the first seven interview subjects, while the other two were each from different universities. The pseudonyms I chose for each subject, as well as his or her educational background and relevant teaching experience, are listed in Table 4.

\section{Interview Subjects and Consent}

Once I had decided that I wished the bulk of my first series of interviews to come from a particular university, an initial list of possible interview subjects was generated based on the potential subjects' research areas. I wished to include subjects whose research falls in the broad category of "Applied Mathematics," as I believed that mathematicians with this background would be the ones who would have thought about differentials the most and would thus provide the most 


\begin{tabular}{|c|c|c|c|c|c|}
\hline \multicolumn{6}{|c|}{$\begin{array}{l}\text { Table } 4 \\
\text { Interview Subjects' Information }\end{array}$} \\
\hline \multirow{2}{*}{\multicolumn{2}{|c|}{ Pseudonym $\quad$ Degrees and Education }} & \multicolumn{4}{|c|}{ Courses Taught } \\
\hline & & 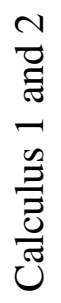 & 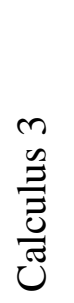 & कิ & 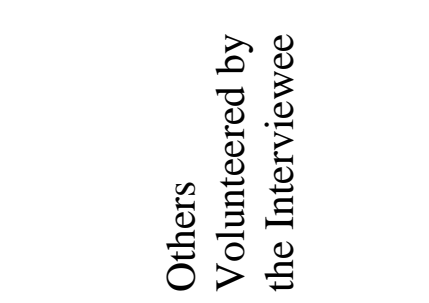 \\
\hline André & $\begin{array}{l}\text { Theoretical and Nuclear Physics, } \\
\text { Nuclear Theory and } \\
\text { Bioinformatics }\end{array}$ & $\checkmark$ & $\checkmark$ & & $\begin{array}{l}\text { Graduate courses in } \\
\text { Advanced Calculus and } \\
\text { Numerical Methods }\end{array}$ \\
\hline Bryan & $\begin{array}{l}\text { Minor in Physics, Ph.D. in } \\
\text { Applied Mathematics }\end{array}$ & $\checkmark$ & $\checkmark$ & $\checkmark$ & $\begin{array}{l}\text { Graduate courses in } \\
\text { Calculus, Differential } \\
\text { Equations, and Modeling }\end{array}$ \\
\hline Christopher & Physics and Applied Mathematics & $\checkmark$ & $\checkmark$ & $\checkmark$ & \\
\hline Diane & $\begin{array}{l}\text { Mathematics and Computer } \\
\text { Science, Ph.D in RUME }\end{array}$ & $\checkmark$ & $\checkmark$ & & \\
\hline Eugene & Discrete Mathematics & & $\checkmark$ & $\checkmark$ & \\
\hline Francis & $\begin{array}{l}\text { Engineering Physics and Applied } \\
\text { Mathematics, Ph.D in RUME }\end{array}$ & $\checkmark$ & $\checkmark$ & & Vector Calculus \\
\hline Gustav & Data Not Collected ${ }^{8}$ & $\checkmark$ & $\checkmark$ & & \\
\hline Xavier & Data Not Collected ${ }^{9}$ & & $\checkmark$ & $\checkmark$ & $\begin{array}{l}\text { Graduate courses in } \\
\text { Analysis }\end{array}$ \\
\hline Yanick & Mathematics, Ph.D. in RUME & $\checkmark$ & $\checkmark$ & $\checkmark$ & \\
\hline Zaphod & Data Not Collected ${ }^{9}$ & $\checkmark$ & $\checkmark$ & $\checkmark$ & Real Analysis \\
\hline
\end{tabular}

interesting results. Once I had a list of applied mathematicians to contact, I added to this list a few other mathematicians from this university whose research areas could be described as "Pure Mathematics" or "RUME" (Research in Undergraduate Mathematics Education) for balance. Emails were sent to the faculty members on this list, and interviews were conducted with those

\footnotetext{
${ }^{8}$ Gustav's interview had to be completed in a smaller time window than the other interviews. The questions asking for this information were among the ones that had to be cut for time.

${ }^{9}$ Xavier and Zaphod listed the schools that they attended, but not their majors. I forgot to ask for clarification before the interview ended.
} 
who responded (subjects André through Diane). After these initial interviews were completed, I wished to ensure a better balance between individuals from the three broad research areas named above, and thus created a second interview list to address imbalances, creating a list with primarily "Pure" mathematicians and RUME mathematicians. Emails were sent to the people on this second list, and interviews were conducted with those who responded (specifically, subjects Eugene through Gustav).

In order to verify the results gathered from the first round of interviews, three additional interviews were conducted. I began my search for interview subjects by sending emails to ten additional faculty of the same university, and only Xavier responded. Since no one else at this university expressed any interest in sitting for an interview, I decided to send requests for interviews to other universities. Initially, I prioritized convenience, and sent a total of fifteen emails to selected faculty at four schools located within an hour's drive from where I live. Only Zaphod responded to my request, and only after a few weeks had passed.

In the time between Xavier's and Zaphod's responses, I chose the additional schools from which I would attempt to select other interview subjects by using Eric Hsu's Spreadsheet of North American Doctoral Programs in Math Education (Hsu, 2013). My justification for using this list was my belief that schools with strong RUME ties might have faculty who would be more willing to participate in a mathematics education research interview. From this spreadsheet, I selected schools, went to their mathematics departments' internet pages, and sent emails to all faculty who had RUME experience and/or seemed to teach first- and second-year calculus often. Yanick responded from the third school to which I sent these emails. As will be shown later, the results of these three interviews supported the framework shaped by the initial seven interviews. Thus, it was determined that no further interviews were required. 
Seven of the ten interviews were conducted in person and in the interview subjects' offices. For these in-person interviews, I presented consent forms to the subjects at the beginning of the interview, and they were given an opportunity to ask questions before the interview began. A copy of this consent form can be found in Appendix C. I video-recorded each interview and collected any work (figures, expressions, or equations) created by the subjects during the interview. André, Yanick, and Zaphod were not able to be interviewed in person, so their interviews were given over computer via Skype and recorded using OBS Software. Before these Skype interviews began, consent forms were emailed to the interview subjects. These forms were signed and scanned copies of the signed forms were emailed to me before each of these interviews began. Only André created diagrams and expressions during his interview, and after his interview's conclusion, he emailed scans of this work to me.

\section{Interview Protocol}

The final version of the protocol that I used for my dissertation interviews is listed in Appendix D and summarized below. All questions in this protocol were meant to be asked, although some interviews did not contain all of these questions due to time constraints. All interviews contained follow-up questions that I asked whenever I needed to have the interview subjects either clarify remarks that I did not initially understand or expand on remarks that I found particularly interesting. Including these follow-up questions, this protocol resulted in interviews that averaged about forty-five minutes in length.

Introductory questions. There were two introductory questions in the protocol. The first asked the subject for his or her academic credentials and teaching history. I requested this information because I thought it possible that the degrees they earned and the classes they taught could be used as categories to help parse the data. The second introductory question (and the first 
"real" question of the interview) was "What does the word 'differential' mean to you?" I believed that since this question asked about differentials without any context, the answers I received could serve as baselines with which to compare the interview subjects' future answers, which would involve differentials presented within contexts.

The questions containing differentials. Most of the remaining questions were about the subjects' conceptualizations of differentials found in various situations. I divided these various situations into two categories. The first category was a collection of the following mathematical expressions containing differentials, numbered here as they were numbered in the protocol:

(1) the Leibniz derivative notation $\frac{d y}{d x}$,

(2) the generic definite integral $\int_{a}^{b} f(x) d x$,

(3) the generic indefinite integral $\int g(x) d x$,

(4) the generic double integral $\int_{0}^{1} \int_{2}^{3} f(x, y) d y d x$, and

(5) the expression $d y=2 x d x$.

For each of these, the subjects were shown the expression, asked how they viewed the entire expression, asked specifically how they viewed the differentials in the expression, and asked whether they thought the differentials in the expressions had a graphical representation and/or a size. As mentioned before, follow-up questions were asked as needed in order to get as complete a picture of the interview subjects' concept images as possible.

The second category of expressions and contexts used some of the same differentialcontaining expressions, but these expressions were placed within a specific context. As numbered in the protocol, these expressions and contexts were: 
(6) the expression $\frac{d \tau}{d t}$ which was contained within the separable ordinary differential equation $\frac{d \tau}{d t}=-k \tau$

(7) the definite integral $\int_{0}^{50} 700-3 x d x$ which was given as part of a problem that asked for the amount of work involved lifting a weight up the side of a building, and

(8) the expression $d u=\frac{1}{2 \sqrt{t}} d t$, which was one of the substitutions needed to evaluate the integral $\int_{1}^{4} \frac{\cos \sqrt{t}}{2 \sqrt{t}} d t$

As before, the interview subjects were presented with these contexts and asked how they perceived the differentials in each of them, with follow-up questions asked as needed for elaboration. These specific contexts were chosen because they contained similar notations (Leibniz derivative notation, definite integrals, and "differential of a function" notation) to the expressions already discussed, and I wanted to see how similar the answers would be when the same notations were presented first without and then within a particular context.

All of these expressions were presented to all interview subjects, and for most of them, were asked in the order listed above. The only deviations were in Gustav's and Zaphod's interviews: when presented with expression (5) $d y=2 x d x$, both of them spoke so much of integration by substitution that I decided to present my "integration by substitution" expression (8), $d u=\frac{1}{2 \sqrt{t}} d t$, immediately afterwards. Following these deviations, we proceeded to the separable ODE (6) and "Work" problem (7), in the proper order.

Two ancillary sets of questions. There were two other sets of questions that were not scripted to be asked at a particular time during the interview, but were asked whenever certain conditions were met. The first condition was the first mention of the word "Delta" by the interview subject. After this mention, and as soon as it seemed feasible, I asked a series of questions designed 
to compare how the subject viewed a $\Delta x$ and a $d x$. These particular questions usually occurred during the discussions of Leibniz derivative notation or definite integrals, as these were topics that seemed to lend themselves to natural discussions of "Deltas." The only person to whom these questions were not presented was Eugene, partly because of time constraints, but partly because he never mentioned the word 'Delta' in his interview.

The second condition that resulted in a set of ancillary questions was the first time an interview subject used a particular phrase that seemed to define a degree of "smallness". Examples of these include "infinitely small" and "infinitesimally small." After an expression like this was used, and as soon as it seemed feasible, I asked a series of questions with the intention of clarifying and possibly quantifying their phrase. Was the phrase they used simply a figure of speech that was not meant to represent anything specific, or did they have an actual value in mind for this particular smallness?

\section{Data Analysis}

Even though I already had a list of differential conceptualizations from my literature review, I did not want to use that list as a basis for this dissertation for two reasons: there was no guarantee that my list was compete and that list consisted mostly of student conceptualizations of differentials from definite integrals. I wanted to generate my own list strictly from the data that I had collected.

\section{Thematic Analysis}

Data were analyzed using Braun and Clarke's (2006) thematic analysis. I chose this particular method in lieu of other grounded theory methods because Braun and Clarke's thematic analysis is a very malleable method. At the time I was beginning my dissertation, I had already conducted two prior studies and reviewed literature that discussed differentials, and all of this work 
made me aware of and familiar with a range of already-existing conceptualizations. Because I now possessed this awareness, I felt that using open coding from Strauss and Corbin's (1997) grounded theory would not be possible. In Strauss and Corbin's open coding, the themes and codes that emerge during the research are solely generated from the data and not the researcher's preconceived notions. Braun and Clarke (2006), however, allow for choices in how one wishes to conduct one's thematic analysis. One of these choices is between conducting an inductive analysis, which is meant to be purely data-driven, or a theoretical analysis, in which analysis is done with particular theories or ideas in mind. Since I had preconceived notions of possible differential conceptualizations from the literature, it seemed a proper choice to analyze my data using thematic analysis.

As mentioned before, I created my list of themes from the data obtained from the first seven interviews. Thus, all of the processes described in the following sections were processes applied to only the first seven interviews (André's through Gustav's). The final three interviews were used to ensure that results from the first seven interviews also applied to new data. Below, I describe the steps that I took to get a list of initial themes and the modifications and rationalizations I made to arrive at the final list of themes.

Coding Data Points. To begin my analysis, transcriptions were made of each interview. These transcriptions contained not only the words spoken by the subject and myself, but also any diagrams or figures drawn by the subject, which were scanned and inserted into the transcriptions. After transcribing all of the interviews, I read these transcriptions a few times in order to become familiar with the data in a general way.

After transcribing, I went through each transcript and made a list of data points, which consisted of any word or phrase that addressed differentials in any way, or any word or phrase that 
seemed interesting and worthy of study even if it did not address differentials. Because it was impossible to tell at this early stage what data might or might not be relevant, I erred on the side of caution and created data points for any text that contained even the smallest hint of relevance or interest. Thus, many data points were created: the average number of data points in each interview was 145, with the most coming from Bryan's interview (167) and the least from Gustav's (126). Two examples of data points taken from André's interview are given in Figure 3.

The process I used to build these data points into themes was a process of refinement similar to the one given in Braun and Clarke (2006). The goal for each step was to begin with a relatively large number of the current type of data element and end with all of these initial data elements organized and arranged into a relatively smaller number of collections of similar elements. For my data, this step was conducted two times: first, my hundreds of data points were organized into dozens of categories, and then this collection of categories was condensed and organized into a smaller list of thirty-seven initial themes.

The initial categorization of my data points began after transcribing the first seven interviews. To give an example of this categorization, while the data points shown in Figure 3 were taken from two different places in André's interview, they described the similar idea that people might interpret differentials in various ways depending on the context in which the differentials were presented ("math versus physics" in the first excerpt and "practical versus historical" in the second). Further analysis of André's interview found many other instances where he stated that differential interpretations can be context-dependent. I went through each of the first seven interviews and created categories by grouping data points that espoused similar ideas under one heading. The result of this work was seven lists of categories that distilled the hundreds of data points into a minimum of fourteen (André and Diane) and maximum of twenty-four (Bryan) 


\section{1) Very, very small change in $x$ \\ 2) "Smaller than anything else $x$ might do" \\ 3) You can do algebra [with them] \\ 4) This is an "Engineering way" to teach calculus \\ 5) "Mathy way": $\epsilon-\delta$}

André: Yes, so they call them, you know, just infinitely small. So just, just imagine $d x$ is a really, really small number, you know, very, very small change in $x$, which is smaller than anything, anything else $x$ might do. And then we do these things - and then it's always handy, you can, you know, you can do simplifications, you can do algebra, and that's how they used to teach Calculus back - that was the "engineering way" of teaching Calculus. And then the "mathy way" was the epsilondelta. And in Romania, when I went to school, we learned epsilon-delta in high school. So we had Calculus in - starting from $11^{\text {th }}$ grade. So we basically had Calc 1 in $11^{\text {th }}$ grade, Calc 2 in $12^{\text {th }}$ grade. And it was epsilon-delta Calc 2 and Calculus. Not bad, OK, but - things that actually you guys do in Real Analysis - and for that famous entrance exam,

[28:27-29:10 is a story about how he blew off integration in high school and had to self-teach it before his exam]

6) Math class: $d x$ is notation 7) Physics class: $d x$ is a thing

(29:11) TM: So your training was that the $d x$ was an entity and not, as you described it, "the symbol is just notation?" So in math class, in math class it was just notation and in physics class it was a thing.

(40:40) TM: Yes, that is clear. Then, what are the roles, to you, of the

58) Same as in single integral

59) Practical role: variable indicator

60) Historical: comes from

$\Delta x, \Delta y$ in Riemann sum

61) He prefers the practical interpretation $d y$ and $d x$ in this expression? Same as in - well, again, same as in in the previous, the single integral, it's - the practical role is that it just tells you what you're integrating with respect to. And, as an historical or kind of symbolic thing here, it represents that it comes from the $\Delta x$ and the $\Delta y$ in a Riemann Sum. Do you have a personal attachment to one of those two above the other? Well, the first one makes sense. The first one is, is, is practical [laughs] and it carries information. The other one is more like a [tails off]

Figure 3: Two examples of the selection of data points from André's interview. The right column contains excerpts from André's interview and the left column contains the data points I coded from these excerpts. ("TM" is the author/interviewer)

categories.

At the conclusion of this process, it became apparent to me that many interview subjects referenced the same or similar categories. Noting the similarities in many categories, I created a thematic map for my data points and categories by going through these seven lists and performing the following algorithm: if the current category on my list seemed related to one that I had already included in my thematic map, I wrote the current category close to the already-included category and drew lines or arrows to show the connection between the two. If the current category seemed 
unrelated to all the categories that had already been placed in my thematic map, I thought of a title that might best describe this category, and wrote this title and the particular category in a new spot on my thematic map. The end result was a thematic map five pages long, with a list of thirty-seven initial themes. Figure 4 shows the portion of my thematic map that discusses this idea that a differential conceptualization might depend on the context in which it resides.

The end result of my thematic map was a list of thirty-seven initial themes shown in Figure 5. As I analyzed this initial list of themes, I realized that there were two ways that this list could be shortened. First, some of the themes on this list were not relevant to the research questions. For example, Initial Theme \#14 encompassed the collection of statements made by my interview subjects who mentioned that $\Delta x$ is a real number. This is a trivial statement with which I would expect all mathematicians would agree, and thus would provide no opportunity for conflict. It is also a statement that does not involve differentials, which are the focal point of this dissertation.

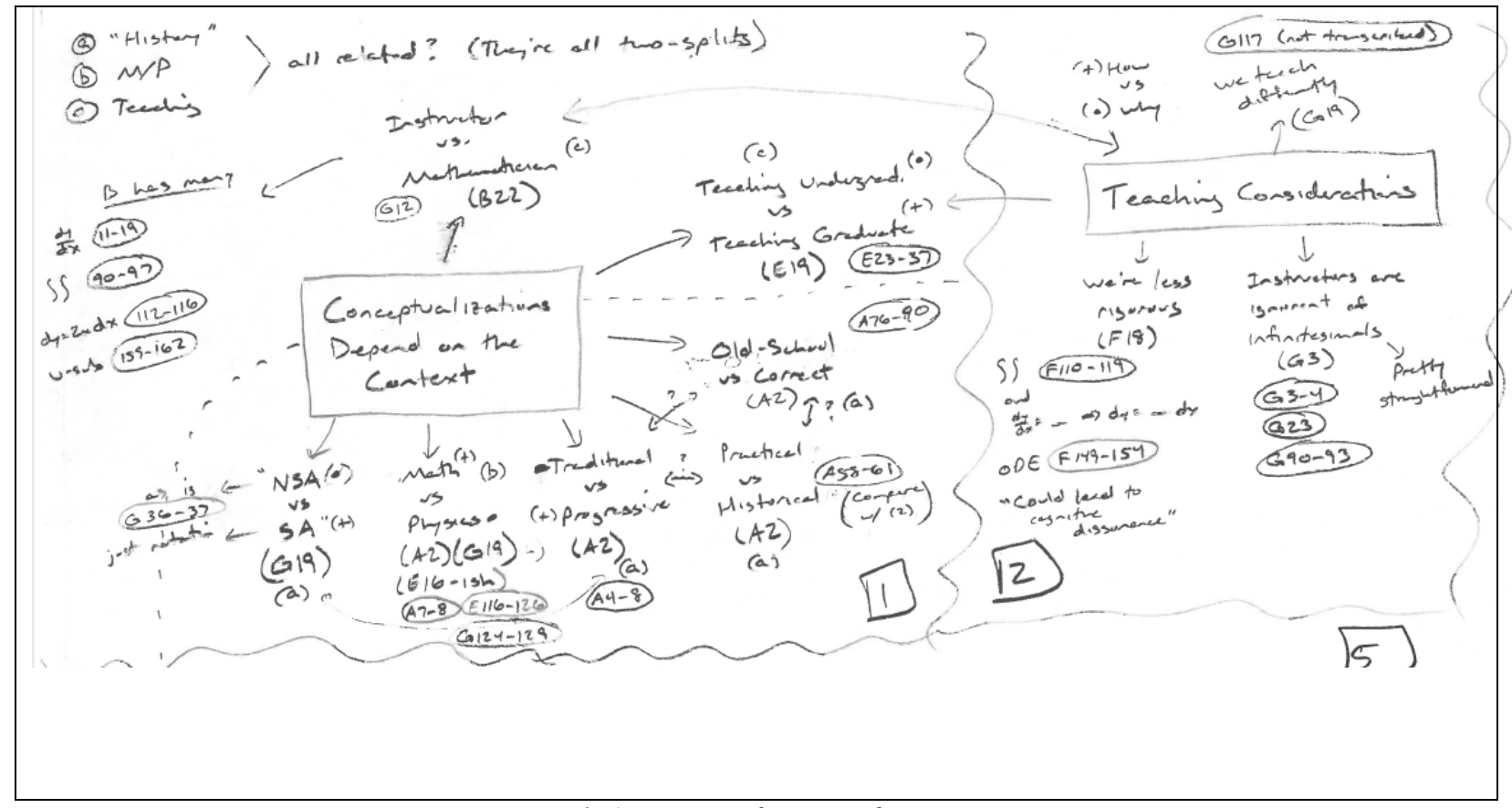

Figure 4: An excerpt from my thematic map 


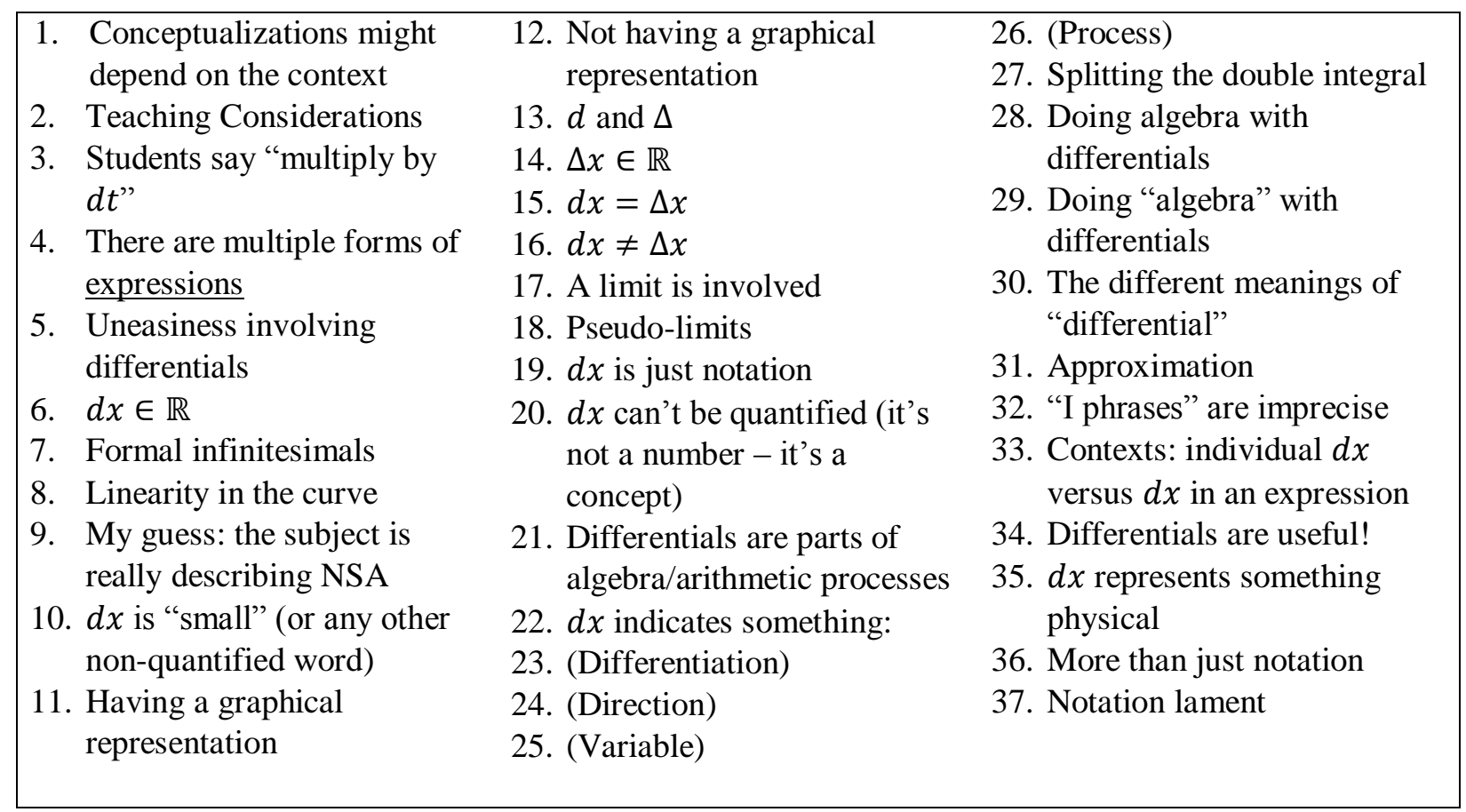

Figure 5: The initial list of themes (from the thematic map)

Thus, this theme was discarded. Second, I combined themes that were very similar to one another. For example, Initial Themes \#3, \#21, \#28, and \#29 each involved some version of the idea that algebraic manipulations might or might not be able to be performed with differentials. These four initial themes were combined into one overall "Algebra with Differentials" theme.

Turning the list of initial themes into a smaller, final list of themes was a multi-step process of elimination, comparison, combination, and refinement. After discarding the irrelevant themes and combining the similar ones, I reviewed my code lists and theme map, comparing them to the updated theme list. If I found any codes or elements of my theme map that did not seem to be fully described in the updated theme list, I refined the theme list to include the missing information. I made nine such updates to my theme list before I felt that my list encompassed the entire data set. To test this, I read through all of the transcriptions one final time, making sure that all of the statements made by my interview subjects could be found in my theme list. The result of all of these machinations was the list of themes given in Figure 6. 


\begin{tabular}{|c|c|c|}
\hline Tier 1: The "Sized" & Tier 1: The "Un-sized" Themes & Tier 2: The Others \\
\hline Themes & 17. The $d x$ arises from a limit & 1. Conceptualizations \\
\hline 6. $\quad d x \in \mathbb{R}$ & 20. The $d x$ is part of an automatic & might depend on the \\
\hline 7. Formal infinitesimals & process $-\mathrm{a}$ process that is done & context \\
\hline $\begin{array}{l}\text { 10. The } d x \text { is only described } \\
\text { as "small" (or any other }\end{array}$ & $\begin{array}{l}\text { without explanation or thought } \\
\text { (usually } u \text {-substitution or solving }\end{array}$ & $\begin{array}{l}\text { 5. Subject uneasiness with } \\
\text { differentials }\end{array}$ \\
\hline non-quantified word) & separable ODEs) & 28. Doing algebra with \\
\hline & 25. The $d x$ indicates a variable or a & $\begin{array}{l}\text { differentials } \\
\text { Ding "alohro" with }\end{array}$ \\
\hline & 26. The $d x$ indicates some other type of & differentials \\
\hline & & 34. Differentials are useful! \\
\hline
\end{tabular}

Figure 6: The first attempt at a final list of themes (main themes only; no sub-themes)

At first, this list of themes seemed to serve me well. I liked how they seemed to coalesce naturally into what I called two "tiers": Tier 1, which included themes that described the differentials directly, and Tier 2, which included themes that described ideas surrounding one's beliefs and uses of differentials. Within Tier 1, I liked what seemed to be a splitting of these themes into a group which appeared to endow the differentials with a size and a group that did not. At the time of its creation, this list seemed like it had clear, objectively-defined categories into which all interpretations of differentials, including ones that would be found in future interviews, would fall. In practice, this turned out not to be the case.

The Tier 2 themes worked well and presented no problems, but when presenting and discussing my results with my dissertation advisor, a previously-unseen problem with the "Tier 1" themes in this list emerged: the existence of overlapping categories. To give one example, Theme \#10 said that the differential was a level of "small" that was unquantifiable, and Theme \#17 said that the differential was the result of a limit process. It was certainly possible that these ideas could be intertwined: a mathematician could view a differential as a small unquantified amount (Theme \#10) that resulted from taking the limit (Theme \#17). I believed that, even with these overlaps that needed to be resolved, the idea that my themes could be partitioned into two tiers was still viable. 
Thus, I left my final list of "Tier 2" themes alone and concentrated on fixing the overlaps in my list of "Tier 1" themes. Since the presence or absence of limits in the creation of a differential seemed important to one's differential interpretation, I decided to make this presence or absence of limits the first criterion by which I would split these themes into new categories. I believed that this bifurcation would be helpful in accounting for some of the overlaps described in the previous paragraphs. For example, this criterion could create a difference between a "small" amount that was created via a limit and a "small" amount that was not.

The second new partitioning of the themes was motivated by the varying degrees of ambiguity that were attached to the idea of "smallness." Not only did the interview subjects describe differences in the sizes of the measurable differentials (a real number versus a hyperreal infinitesimal number versus some unquantifiable idea of "small"), but there also appeared to be differences in the level of "numerical tangibility" certain differentials might possess. For example, one might suggest that a differential that is described as an actual, measurable quantity is imbued with an idea of "numerical tangibility," a differential that exists as an object but without any numerical qualities is imbued with a lesser degree of "tangibility", and a differential that exists merely a variable indicator might be more "ephemeral" than the other differentials would be. It seemed to me that one could create a continuum of differential descriptions, with "ephemeral and not a concrete object" on one side and "actual, measurable, well-defined value" on the other.

These ideas suggested to me that a flowchart would be the best way to present my refined final list of what used to be my "Tier 1" themes. With a flowchart, I could begin by splitting the themes into two separate directions, based on the presence or absence of a limit process in the differential's description. Once this split has occurred, I could proceed through a series of decisions based on the continuum described above: start by determining if the differential is ephemeral and 
without tangibility of any kind. If so, stop and assign an "ephemeral" theme to that differential. If not, then proceed through decisions that ask if the differential has more and more "tangibility," either stopping if a certain level of tangibility is reached, or proceeding until the final step of "actual, measurable, well-defined value" is reached. Because this flowchart was the culmination of my analysis of my Tier 1 themes, I show it in Figure 7, but I save a more-detailed look at this flowchart and this final list of themes for the next chapter of this dissertation.

Data analysis for the second round of interviews. Since the second round of interviews was primarily conducted to test the efficacy of my theme lists, data analysis for these interviews was done by a more-abbreviated version of my previous thematic analysis. While I did identify and code data points in the transcriptions of these three interviews, I did not do so with the creation of the lists and thematic maps shown in Figures 2 through 4. Instead, I noted the presence of data points in each interview, and analyzed them with my flowchart and theme lists in mind: could each description of a differential be easily categorized into a Tier 1 or Tier 2 theme, and if the former, would this description fit into my flowchart as it currently existed?

The concept images from each interview subject will be discussed in the next section of this dissertation. For now, it is sufficient to say that, while these last three interview subjects described subthemes that were not present during the first seven interviews, their themes were easily categorized into the flowchart and tiers I had already established. To me, this suggested two things. First, the overall idea of the flowchart was sound: a system in which differentials are categorized by whether they arose from a limit and their degree of "numerical tangibility" seems to be a system that can account for any differential conceptualization. Second, since individual concept images can vary wildly, it is highly probable that each space on the flowchart and the Tier 2 list, can have many subthemes. 


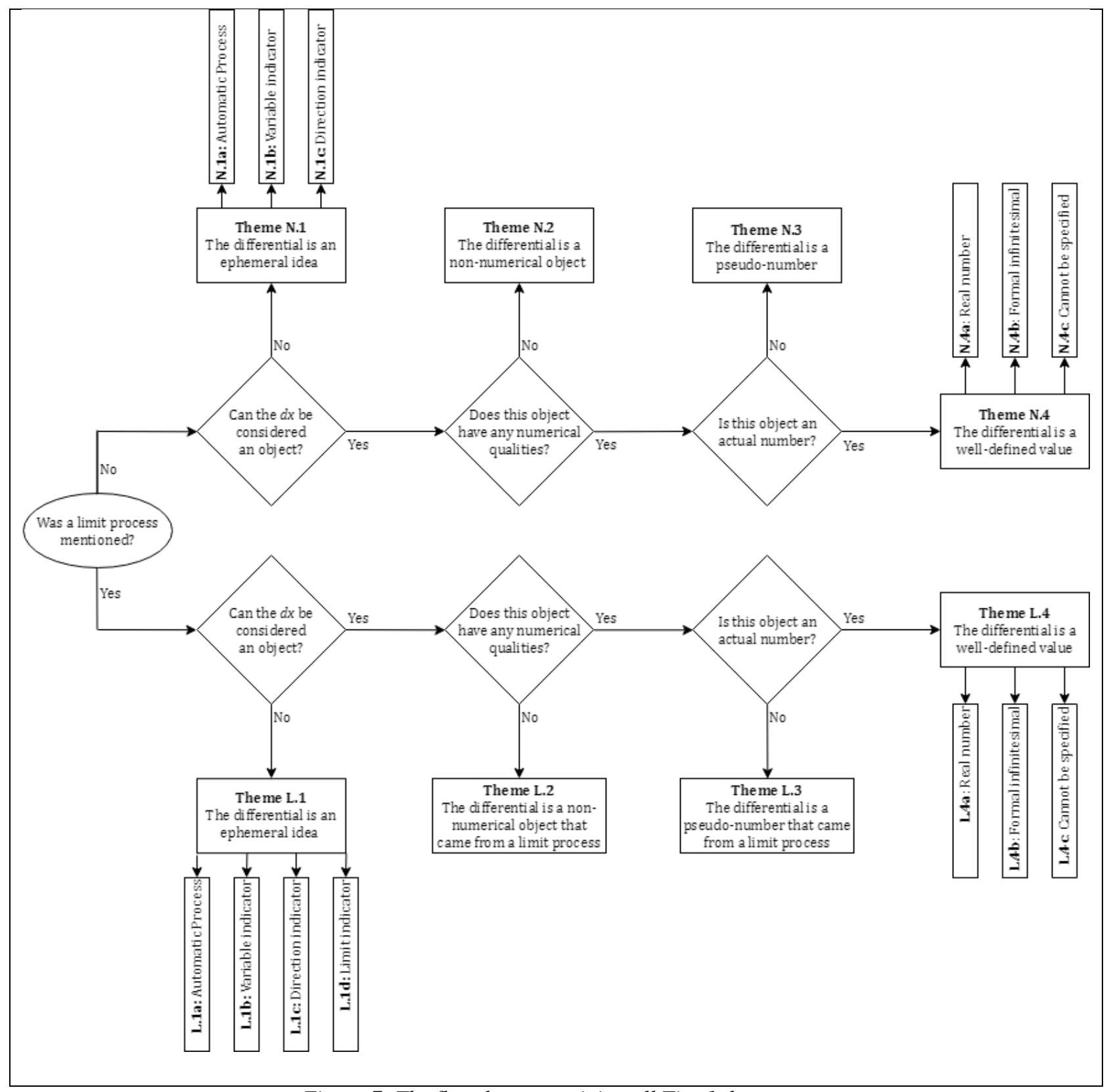

Figure 7: The flowchart containing all Tier 1 themes

My decision to end data collection after the last three interviews, and ten in total, was motivated by these two ideas. The last three interviews suggested that, no matter what conceptualizations might arise in future research, there would be a spot for them in my flowchart, as long as the person conducting this research will be willing to accept that there probably exist subthemes and Tier 2 themes hitherto undiscovered. Believing that it might be impossible to 
explore every Tier 2 theme and flowchart subtheme and that no further interviews would give conceptualizations that would not fit in my flowchart, I suspended data collection and analysis.

\section{Visual Representations of Data}

As mentioned in Chapter 5, when I found a data point, I wrote a brief description of it in the margin of the interview transcript. This was an acceptable way to list the data points initially, but resulted in over a hundred data points per interview spread out over multiple pages. I believed that it would be difficult to both analyze the themes from an entire interview and compare themes from different interviews if all I used for these analyses and comparisons were these notes. Thus, I created two types of visual representations of the themes I found in each interview. For me, seeing themes grouped by positions on a flowchart or differentiated by combinations of colors allowed me to make connections and see trends in an easier way than when I tried to make connections and see trends by only reading transcripts with notes and theme names scrawled in the margins.

The first visual media I created were specific flowcharts for each interview subject and for each item in my interview protocol. For the "interview subject" flowcharts (an example is André's flowchart, shown in Figure 8), I placed numbers or letters keyed to each element of the interview protocol $1^{10}$ next to each theme that was used during the discussion of these elements. If more than one theme was used for an element, I used dashed numbers to indicate the multiple themes. For

\footnotetext{
${ }^{10}$ For the flowcharts of each interview subject, I used the numbers (1) through (8) for each expression (these numbers were attached to each expression in the interview protocol - see pp. 162-166 in Appendix D), and the following letters: (D) for themes discussed during the initial question "What does the word 'differential' mean to you?", $(\Delta)$ for themes presented during the discussion of $d x$ versus $\Delta x$, and $(\mathrm{P})$ for themes conveyed during discussion of their particular "small" phrase)
} 


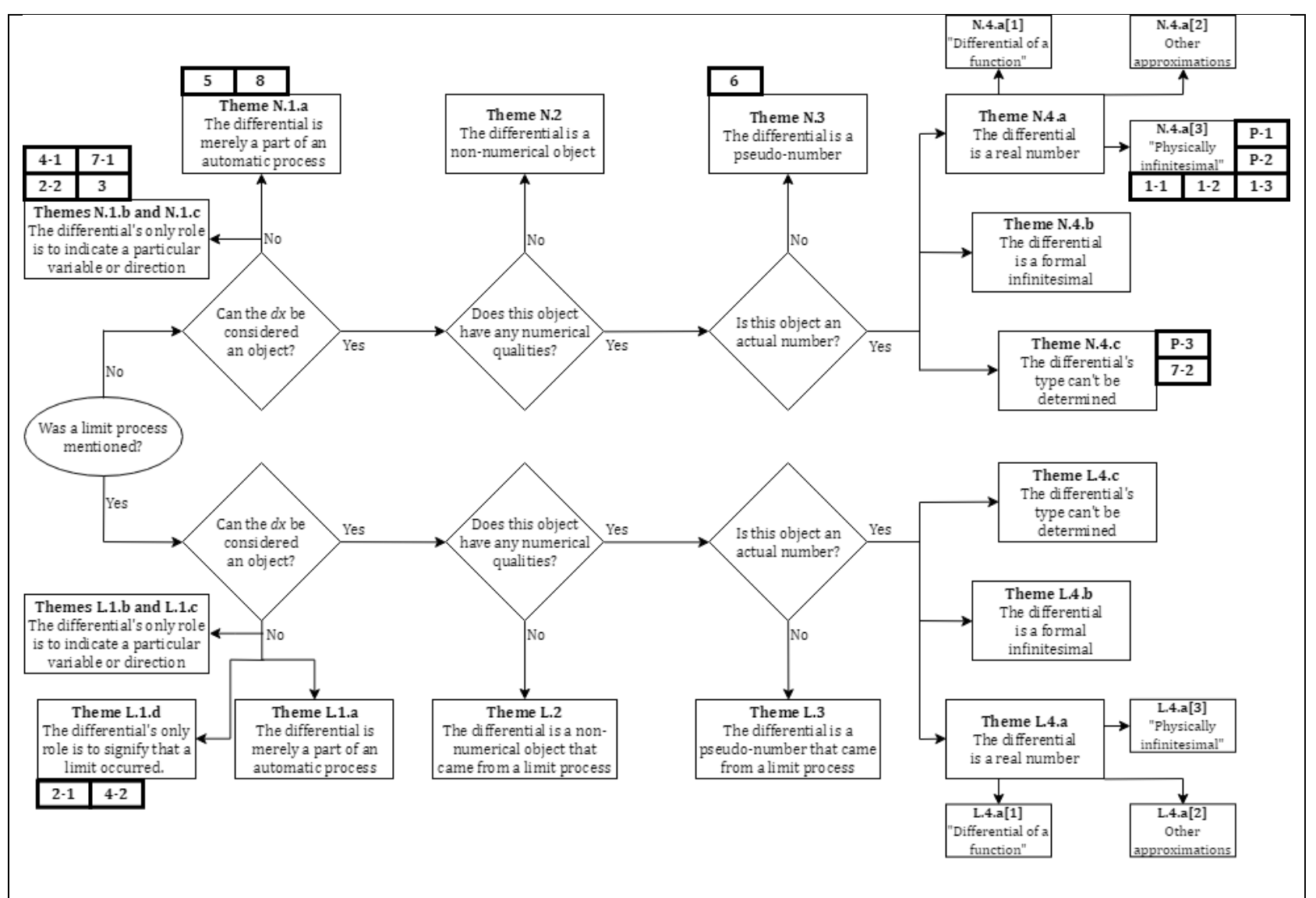

Figure 8: An example of an individual flowchart (André's)

example, one can see that André had two views of the differential in the "Work" problem, which was labelled as (7) in my interview protocol. These two different views were indicated by the "71 " and "7-2" in different positions on his flowchart. For the "items in my interview protocol" flowcharts, I placed the first letter of my interview subject along with similar dashed numbers to indicate multiple themes.

I decided to use Excel spreadsheets for the second medium, and these sheets were created both for each interview subject and for each item on my interview protocol. Within each of the “interview subject” sheets (an example is André's spreadsheet shown in Figure 9), I created columns headed by each item in my interview protocol and placed colored cells under these headings to show the existence of a particular theme. For the "items in my interview protocol" 


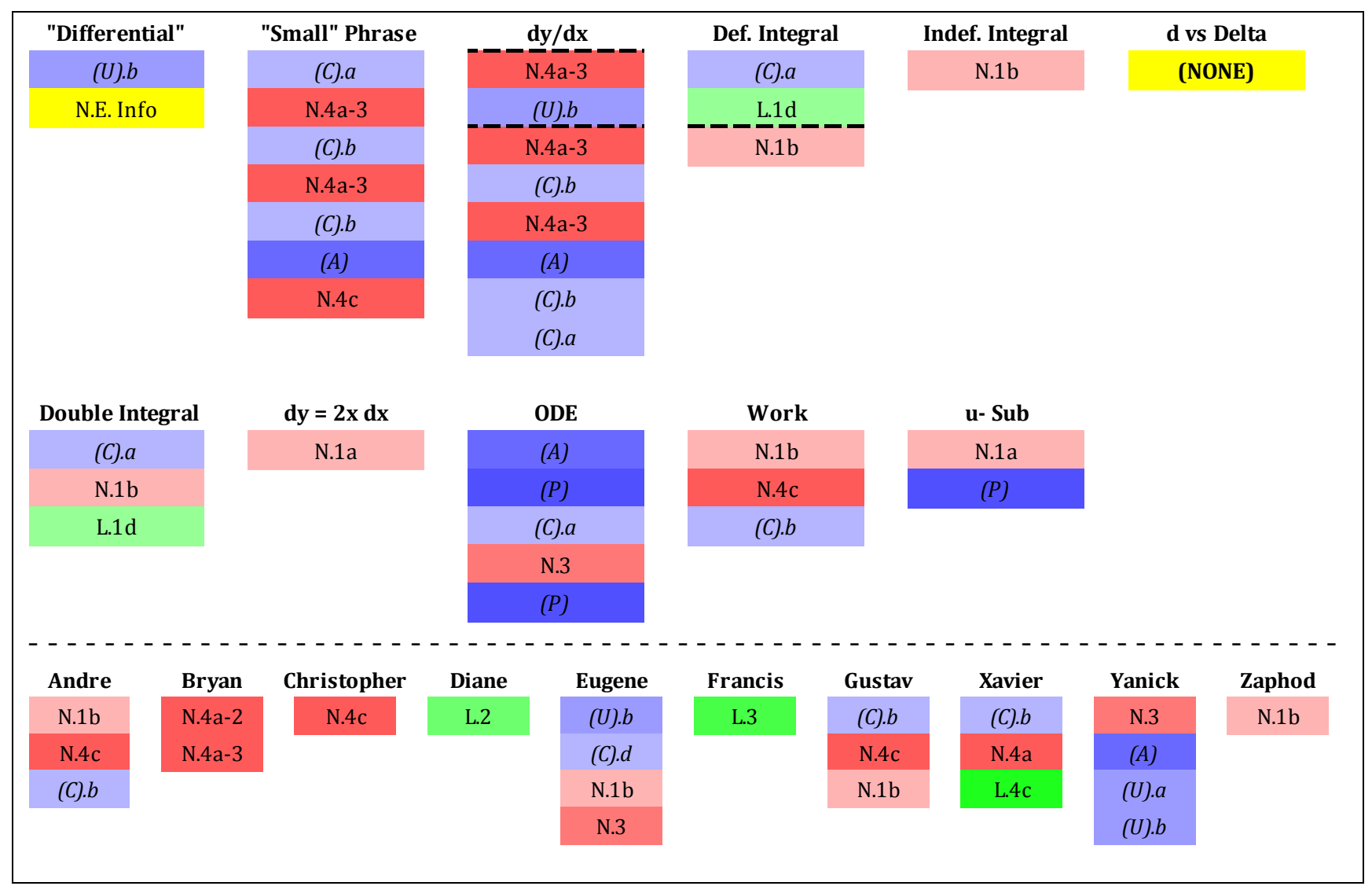

Figure 9: Two examples of spreadsheets (Top: André's, Bottom: The “Work” problem)

sheets (an example is the "Work Problem" sheet, also shown in Figure 9), my column headings were the interview subjects and the columns were copied and pasted from the "interview subject" sheets (For example, to create the " $\frac{d y}{d x}$ ” sheet, I copied everyone's “ $\frac{d y}{d x}$ " column onto one sheet, each person's column under his or her name.) Note that these initial sheets were created using only the first seven interviews, as those were the interviews dedicated to generating the theme lists.

In every sheet, I chose red cells for all non-limit " $\mathrm{N}$ " themes and green cells for all limit "L" themes, shading all of these types of cells from lightest to darkest depending on their position in the "tangibility" continuum. For example, Theme N.1 represented viewing the differential as an automatic process and had the lightest shade of red, while Theme N.4 represented viewing the differential as an actual number and had the darkest. For all Tier 2 themes, I chose blue cells and 
shaded them from lightest to darkest in the order that I will present them in the "Results" section (Theme (C) had the lightest blue, and Themes (P) and (I) had the darkest.) I added yellow cells for instances in which a subject was not discussed or there was not enough information to make any determination $^{11}$, and I added black cells to indicate when either an interview subject claimed that a differential had no meaning or the differential having no meaning could be inferred by his or her words.

I created one cell for each instance of the particular theme being used, and I defined one "instance" as discussing a particular theme without interruption. I chose this definition of "instance" to account for the different personalities and speaking patterns of my interview subjects. One interview subject might be taciturn, and give a one-sentence answer in which a particular theme is mentioned only once, while another interview subject might be more garrulous and give a multi-sentence paragraph in which the same theme is mentioned often. However, this difference in the amount of speech does not necessarily correlate with the strength of the theme in each subjects' concept image. It is possible that the taciturn interview subject has a much stronger attachment to the theme than the garrulous interview subject, and if so, giving the garrulous interview subject more cells in his or her sheet might suggest an incorrect estimation of the strengths of each theme. If two consecutive cells contain the same theme, it is because the interview subject described the same theme in two ways that were different enough to warrant consecutive cells.

\footnotetext{
${ }^{11}$ For example, the second question in the interview was "What does the word differential mean to you?" Most interview subjects replied with some version of the word "small," but since the interview had just begun, there was not enough information at that time to interpret "small" within the framework of my flowchart. Was it a small object? Was it a pseudo-number? A real number?

Consequently, a lot of the "Differential" spots on my Excel sheets received yellow cells.
} 
Horizontal dashed lines denote instances in which the discussion on the current topic was interrupted by the discussion of another topic. For example, in André's interview, he interrupted his remarks on the definite integral to discuss the indefinite integral. The dashed line in André's “Definite Integral" Excel sheet (Figure 8) shows this break. Additional dashed lines were used to denote when the subject discussed differentials in ways that deviated from the interview protocol. For example, some subjects discussed differentials in Chain Rule notation $\left(\frac{d y}{d x}=\frac{d y}{d t} \frac{d t}{d x}\right)$, which was not a notation in my interview protocol. I used dashed lines to separate the themes found in these Chain Rule discussions from the themes found in the course of the interview protocol.

I believe that these flowcharts and spreadsheets provide nuance that the transcripts lack. For example, during discussion of the definite integral there were times in which André said that the $d x$ in a definite integral was both limit-based and non-limit-based. However, when one looks at André's spreadsheet, he or she sees that his "Definite Integral" column contains both the green and red cells that designate limit-based and non-limit-based themes, respectively, but it also contains a blue cell marked (C).a, which designates a "Context" theme, and specifically, a "historical context" theme. This suggests that André's views of the definite integral's differentials are not a simple contradiction, but rather a more nuanced view that limit-based and non-limitbased conceptualizations each have a place in the historical development of integration and integral notation. 


\section{DATA AND RESULTS}

This section condenses and summarizes the responses that I obtained from my interviews which led to my theme lists and framework. For clarity to the readers, I will first describe each theme that resulted from the data analysis as well as the tools that I used to help me visualize the data in a more dynamic way. Then, I will present summaries of each interview subject's responses and all responses for each mathematical expression used in the interview protocol, specifically drawing attention the presence or absence of each of the themes. Finally, I provide answers to the research questions.

\section{The Flowchart and the Final Tier 1 Themes}

I begin this section by showing again the flowchart used for my Tier 1 themes (shown again in Figure 10). These Tier 1 themes are designated by a two-part labelling system. First, each of these theme's labels begins with the letter "L" or "N", depending on whether a $\underline{\mathbf{L}}$ imit process was

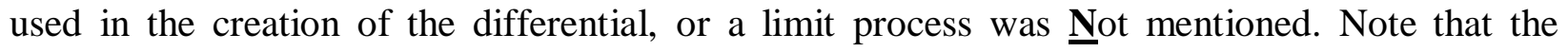
interview subject did not necessarily have to use the word limit to be labeled as a " $\mathrm{L}$ " theme as long as a limit process was described. Next, a number was assigned that represented the theme's position on my "numerical tangibility" continuum: I assigned a "1" if the differential did not seem to be a concrete object, " 2 " if it seemed to be a concrete object but without possessing any numerical qualities, " 3 " if the differential seemed to be an object with numerical qualities but was not described as an actual, quantifiable number (I will call such non-numerical objects "pseudonumbers" throughout this dissertation,) and "4" if the differential was a measurable, well-defined number. Some of these themes contain subthemes, which will be discussed below.

\section{Descriptions of Each Tier 1 Theme}

Since my flowchart was based on an idea of "numerical tangibility," I think it more natural 


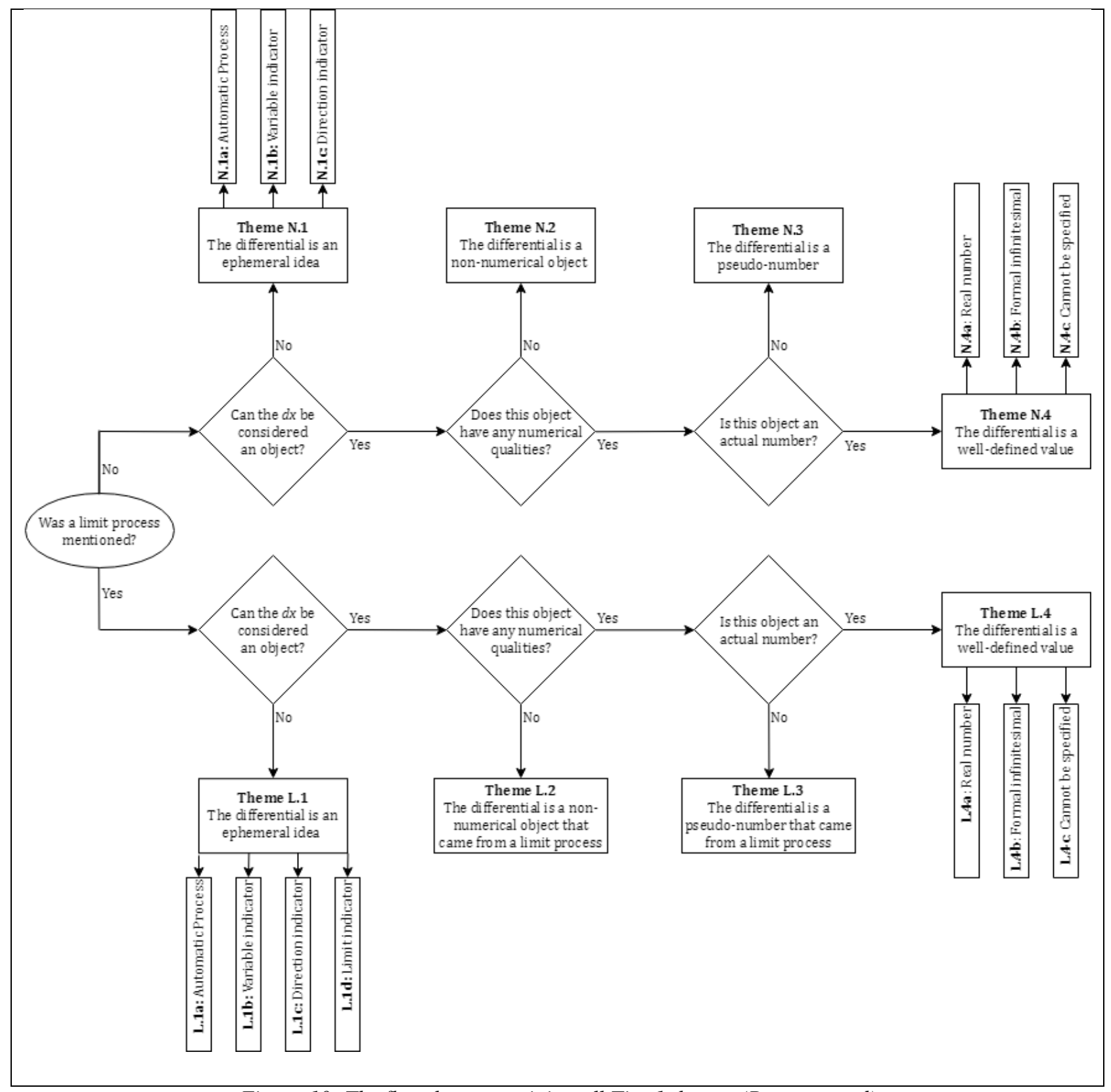

Figure 10: The flowchart containing all Tier 1 themes (Re-presented)

to begin my list of theme descriptions and examples by starting with the actual, well-defined types of numbers with which mathematicians are familiar. Thus, I am going to begin my descriptions with the "4" themes and proceed through my continuum to the " 1 " themes. I will present these descriptions of the Tier 1 themes in pairs. For example, I will present Themes N.4 and L.4 together, N.3 and L.3 together, and so on, since the only difference between an " $\mathrm{L}$ " theme and an " $\mathrm{N}$ " theme 
with the same number is the presence or absence of a limit process in its development. Unless stated otherwise, the reader can assume that the only difference in any two themes presented together is the presence or absence of a limit being used in the creation of a differential.

\section{Themes N.4 and L.4: The differential is a quantifiable or formally-described number.}

In this theme, the idea that the differential has a measurable value was either directly or indirectly stated by the interview subject. This theme was used for differentials defined as real numbers or hyperreal infinitesimal numbers as found in nonstandard analysis, but this theme was also invoked if the interview subject convinced me that the differential has a size which can be defined, even if he or she did not explicitly state whether that size is real or infinitesimal. There are subthemes that explore both these different ideas and the many ways that differentials can be interpreted as real numbers.

Themes N.4a and L.4a: The differential's value is a real number. In this theme, the value of the $d x$ was stated to be a real number. Sometimes this statement was done indirectly, as when someone described the differentials in the "Linear Approximation" context $(\Delta y \approx d y=$ $f^{\prime}(x) d x$, where $\Delta x=d x$.) If someone used this context, then he or she was saying that both $d x$ and $d y$ are real, because $\Delta x$ and $f^{\prime}(x)$ are also real. Sometimes this statement was done directly, as in Bryan's $d x$ in the definite integral: “But I don't think of it as infinitely small. It's always it's always a finite number," and later "Right, [the $d x$ ] would be $10^{-4}$ or something." Sometimes $d x$ was directly described as a real number, but with a definition similar to Courant and John's (1965) "physically infinitesimal". Examples of this were André's "a difference in $x$ that is much smaller than any relevant scale in the problem," and "[The differentials] are very small compared to - not so much to $x$, but more like ... to the scales at which $x$ or $y$ varies significantly." 
Themes N.4b and L.4b: The differential's value is a (formally-defined) infinitesimal. The $d x$ was described as a formally-defined infinitesimal number as found in nonstandard analysis. Again, this could have been stated indirectly or directly. Gustav mentioned Abraham Robinson by name, and, during discussion of what "small" phrases meant to him, said, "Well, I can give you the definition: that's smaller than any $\frac{1}{n}$ and greater than zero. That's [a] kind of positive infinitesimal." This was a fine definition for a hyperreal infinitesimal, but Gustav stopped short of actually describing any of the infinitesimals in his interview in this way. By contrast, Zaphod not only mentioned Robinson but specifically stated during his discussion of Leibniz derivative notation: "so if I'm thinking about differentials as objects with size, I think about them as infinitesimal elements, so I think about them as literal infinitesimals in the Abraham Robinsons sense."

Note that this theme was not being used if the interview subject just happened to use the word "infinitesimal" as a vague, informal descriptor, such as in the phrase "infinitesimally small." Instead, the assignment of this theme required some indication by the interview subject that he or she is familiar with some form of nonstandard analysis and the formal hyperreal infinitesimals that reside therein.

Themes N.4c and L.4c: The differential has a value of unspecified size, inferred to be real or infinitesimal. This subtheme was to be used when the subject assigned the $d x$ qualities that gave it a well-defined size, but the particular type of well-defined size (real or infinitesimal) was not explicitly stated. To give an example, I will use Christopher's phrase "the $d x$ is small enough so that the linear approximation is very accurate." Approximations can be "very accurate" if the value of the $d x$ is a small enough real number. Approximations can also be "very accurate" if the value of the $d x$ is a hyperreal infinitesimal (the approximation will be inaccurate by another 
hyperreal infinitesimal.) Sometimes further questioning enabled me to determine that the subject was conceptualizing the $d x$ as a real number or as a hyperreal infinitesimal, enabling me to assign subtheme N.4a or N.4b. Other times, such as with Christopher's phrase above, the data was not clear enough to make the distinction. I classified these instances as subtheme N.4c.

Themes N.3 and L.3: The differential is a "pseudo-number." In this theme, interview subjects viewed a differential as a concrete object that possessed numerical qualities such as "size" or "width," but these objects were presented more as abstract ideas rather than specific, measurable values. I called such objects "pseudo-numbers" and assigned them a "3" in my "numerical tangibility" continuum. A differential was categorized as a pseudo-number if it was described as a concrete object with numerical qualities but without a clear, direct statement that this differential was a real or infinitesimal number. A differential was also categorized as a pseudo-number if the interview subject hedged his or her description of a particular differential by saying that it is treated "like" it is a number or "as if" it were a number.

To give an example of a limit-based pseudo-number: Diane described most of her differentials in terms of limits, and, indeed, talked about the $d \tau$ and $d t$ in the separable ODE as limits of $\Delta \tau$ and $\Delta t$. But when discussing the solution of the ODE, she said, "The funnier part for me about these kind of problems is that we start treating them like an actual number and start doing algebra with them," and summarized her views on this $d t$ by saying, "It's an infinitely small quantity; it's not really a number, but we think about it that way." This $d t$ arose from a limit, but given that her often-used phrase "infinitely small" was never explicitly quantified by her during her interview, this $d t$ could not be assigned Theme L.4. Instead, I assigned it Theme L.3 because it was treated "like an actual number" when it comes to multiplication, and thus, it is an object with numerical qualities. 
Themes N.2 and L.2: The differential is an object with no numerical qualities. In my data, it was possible that a differential might not be given any descriptors that would classify it as a number, such as "size" or "value," but might still be considered to have some tangibility, and thus, still be a mathematical object.

I understand that there might be an issue of semantics with the word "object." By the strictest of definitions, the definite integral $\int_{1}^{10} x^{2} d x$ could be considered to contain up to eight objects: the integral symbol, the lower bound, the two digits that make up the upper bound, the two "pieces" of $x^{2}$ and the two letters of $d x$. However, I would submit that mathematicians would not consider the " 10 " and the " $x$ " in this context to be made up of two pieces. I would also surmise that when a mathematician sees this $x^{2}$, a part of his or her evoked concept image would be a picture of a parabola, or the graph of a parabola on the Cartesian plane, ensuring that mathematicians have no trouble viewing $f(x)=x^{2}$ or other functions as concrete, tangible, mathematical objects.

The assignment of the number " 2 " to a differential in my framework would be an understanding from the interview subject that the differential was afforded a similar level of tangibility. This tangibility could have been ascertained by the interview subject describing differentials as objects worthy of study, or by the interview subject describing differentials as having an equal worth to other mathematical objects in an expression. For example, signifying in some way that the $d x$ in the definite integral $\int_{a}^{b} f(x) d x$ is an "equal partner" to $f(x)$ in this expression and not an afterthought.

To give an example of this: Diane described the differential in her definite integral as "the result of a limit process" and noted that "[The $d x$ in the definite integral] sort of stands in for the $\Delta x$, but I don't think that I'm taking infinitely many points, finding the functional value and 
multiplying by an infinitely small number necessarily." Diane clearly discussed this $d x$ as having a meaning and purpose, cementing it as an "object," in the above sense. But in stating that it cannot be multiplied, she did not assign this object a numerical quality. Thus, this differential received a designation of Theme L.2 instead of Theme L.3.

\section{Themes N.1 and L.1: The differential represents an ephemeral (not concrete) idea.}

Finally, a differential might have been considered as neither a sized quantity nor a non-numerical object. To continue the semantic discussion from the above section, a differential assigned a "1" in my framework is one that was not perceived to be equally tangible as more standard mathematical objects. In this dataset, I found two ways that this occurred: the differential was a part of some automatic process or the differential was an indicator of how to interpret an expression. The subthemes that explore these different ideas are listed below.

Themes N.1a and L.1a: The $d x$ is merely a part of an automatic process. In this subtheme, the differential was merely a part of a process that was reflexively completed without any explanation or thought. For an example of this, Diane, when discussing how the substitution step $d u=\frac{1}{2 \sqrt{t}} d t$ for the integral $\int_{1}^{4} \frac{\cos \sqrt{t}}{2 \sqrt{t}} d t$ is sometimes taught, said:

I don't know how to explain it, it's just that it bothers me when we write this kind of thing down without really explaining it to our students in Calc. 2, I guess. And maybe I'm the only one that doesn't. Maybe the other people are really clear about why we're saying $d u$ and $d t$ and just sticking them there. But I feel like when we teach the $u$-substitution in particular, we just write this $[u=\sqrt{t}]$ and write that $\left[d u=\frac{1}{2 \sqrt{t}} d t\right]$, and we help them solve and plug stuff in, and we don't spend enough time talking about what is $d u$ and what is $d t$. 
While Diane was speaking of teaching in general, the fact that she ended the above quote with "and I'm just as guilty of that," while André noted that he called the same substitution step "just a mnemonic" showed that this subtheme exists in my data set. As for its classification, the fact that no time was given to the discussion of any potential characteristics of the $d u$ and $d t$ while they were being used in this substitution step is what kept them as "ephemeral" (thus, Theme N/L.1) and not classified as "objects" (Theme N/L.2) in my continuum. These differentials cannot possess the same tangibility as other, better-analyzed differentials if they were only automatically written down and immediately discarded once a particular evaluation step was concluded.

Themes N.1b and L.1b: The $d x$ serves merely as a means to indicate a particular variable upon which some process or characteristic is based. In this subtheme, the use of a " $d x$ " in an expression means that " $x$ " is the relevant variable for whatever operation is suggested by the expression. This was a theme that was already mentioned in the literature review as a common student conceptualization of the differential in an integral, and in my data, this theme was predominant in my interview subjects' indefinite integrals and present in their definite integrals. However, I believe that this subtheme was not limited to integration. When seeing the notation “ $\frac{d y}{d x}$, , a few of my interview subjects specifically stated that $y$ was a function of $x$ based on the position of the $y$ and the $x$ in that notation. I would surmise that, in this expression, the differentials indicated the dependent and independent variables, and thus fall under this subtheme.

Regarding the semantics of the word "object" mentioned above, I would put these differentials in the "ephemeral" category (Theme N/L.1) and not in the "object category (Theme N/L.2) because, to me, these differentials seem more akin to verbal directions than tangible mathematical expressions. To put it another way, if one views the $d x$ in a definite integral as only "mentioning the variable of integration," then the $f(x)$ and $d x$ are not equal partners, as they 
would be if the $f(x) d x$ was viewed as a multiplication of two factors. Instead, with this theme, the $d x$ described how the more-important $f(x)$ will be integrated and thus takes on a secondary role.

Themes N.1c and L.1c: The $d x$ indicates a particular direction of "travel." This subtheme, found in only the double integral and definite integral, was similar to the previous subtheme in the sense that the differential only serves as an indicator, but in this case, an idea of movement and/or the word "direction" is specifically given. For example, graphical representations of the double integral $\int_{a}^{b} \int_{c}^{d} f(x, y) d y d x$ can contain the conceptual metaphors of the differentials $d y$ and $d x$ representing "moving in the $y$-direction between $y=c$ and $y=d$ " then "moving in the $x$-direction between $x=a$ and $x=b$." Again, if one viewed these differentials with this conceptualization, then he or she was using them to help describe the integration of the more-important $f(x, y)$, and thus, the differentials would not be considered as "objects" like they would be if viewed as reified limit processes or factors of the product $f(x, y)$. $d y \cdot d x$

Theme L.1d: The $\boldsymbol{d x}$ indicates that a limit occurred. This was another rarely-used subtheme, and was found only in André's interview. In this subtheme, the subject indicated that the notation containing the $d x$ was created by using a limit process. To give examples, when André discussed the definite integral, he noted " $d x$ represents the fact that it was a sum, it was a Riemann sum," and "it represents the fact that the integral is a limit of a sum like this." Instead of representing a specific object, such as the Riemann sum's $\Delta x$, which would warrant the assignation of Theme L.2, André's differentials represented the fact that a limit existed. Thus, they served as indicators of a process, and, like the differentials that served as indicators of a variable, were 
classified under Theme L.1. Since this subtheme is meant to indicate the existence of a limit process and is thus dependent on a limit, there is no corresponding subtheme N.1d.

A brief note about subthemes. Even though I did not find any during my research, I do not wish to suggest that no subthemes can exist for Themes N.3/L.3 and N.2/L.2. A lack of subthemes in this dissertation means only that when these themes appeared in my data, all instances of the same theme were described similarly enough to one another that I thought no subthemes were warranted for this particular data. Similarly, I do not wish to suggest that the subthemes I listed here are the only possible subthemes for Themes N.4/L.4 and N.1/L.1, only that the listed subthemes were the ones that appeared in my data. The vastness and uniqueness of individual concept images suggest that future research could contain new interpretations of these eight main themes, necessitating the introduction of additional subthemes.

\section{Descriptions and Examples of Each Tier 2 Theme and Subtheme}

The themes that I have categorized as "Tier 2" themes are the themes that are not directly tied to one's conceptual understanding of differentials but rather discuss ideas about differentials. Table 5 contains the eight final Tier 2 themes which emerged from the data. In this section, I provide summaries and examples of these themes and some subthemes. Since the first five were the most common and interesting, they will be discussed in greater detail than the others.

\section{Theme (C): The Meaning of the $d x$ Depends on a Particular Context}

The presence of this theme signifies that the interview subject believed that the meaning of the differential can change depending on the context in which the differential is found. Thus, there can be more than one way to interpret the same differential. I designated all of the contexts found in my dataset as subthemes and list them below. I do not claim that all of the contexts listed below are the only contexts which might exist; further research might unearth contexts which my 


\begin{tabular}{|c|c|}
\hline \multicolumn{2}{|c|}{ List of all Tier 2 themes } \\
\hline Symbol & Description \\
\hline (C) & $\begin{array}{l}\text { One's conceptualizations of differentials can depend on the Context in which the } \\
\text { are presented. }\end{array}$ \\
\hline (U) & 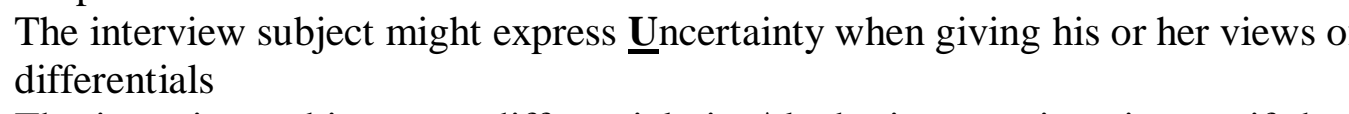 \\
\hline (A) & 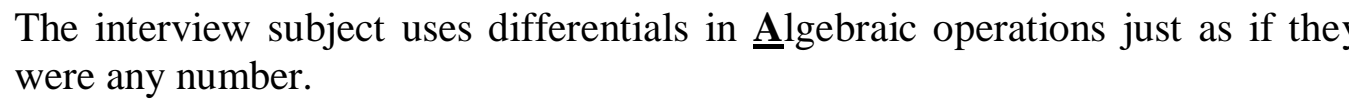 \\
\hline (“A”) & $\begin{array}{l}\text { There is hesitancy when discussing algebraic differentials - I term this them } \\
\text { "Algebra" with differentials }\end{array}$ \\
\hline (A) & $\begin{array}{l}\text { The opposite of Theme (A): one cannot perform } \underline{\text { Algebraic operations witl }} \\
\text { differentials }\end{array}$ \\
\hline$(\mathrm{P}-\mathrm{L})$ & The differential resulted from the use of a $\underline{P}$ seudo-Limit \\
\hline (P) & The interview subject views differentials or differential notation as Practical. \\
\hline (I) & The interview subject views differentials or differential notation as Impractical. \\
\hline
\end{tabular}

interview subjects never considered.

\section{Theme (C).a: The interview subject mentions that the interpretations of differentials}

have changed over the course of history. In this subtheme, the interview subject described the analyses of differentials as being dependent on a particular time in history. In my dataset, this theme was used by André and Gustav. Gustav prefaced some of his descriptions of differentials by noting the historical development of analysis from Leibniz through Cauchy to Robinson. Specifically, he called the differentials in the Leibniz notations of derivative and definite integral "historical leftovers," but also "notations based on intuitions," where these "intuitions" referenced Leibniz' informal understanding of infinitesimals. André used terms like "traditional" and "old school" in some of his descriptions, and defined the differentials in the double integral as follows:

"The practical role is that it just tells you what you're integrating with respect to. And, as an historical or kind of symbolic thing here, it represents that it comes from the $\Delta x$ and the $\Delta y$ in a Riemann Sum," showing that, for him, a "historical" context changes the differential's meaning. 


\section{Theme (C).b: Differentials might be viewed differently by mathematicians and other}

experts. This subtheme was used when the interview subject mentioned any idea that mathematicians and other experts view certain differentials in different ways. Note that while my literature review discussed physics education research, this theme would also hold for engineers or members of other "applied" fields. This theme was most prevalent in the "Work" problem, where multiple interview subjects (André, Eugene, Gustav, and Xavier) described the integral similarly: the integral was initially formed in a "physics" context, in which the $d x$ represented a small amount of wire or distance. Once this integral was constructed and needed to be evaluated, one would switch one's view to a "mathematical" context, in which the $d x$ then indicated the variable of integration.

Outside of the "Work" problem, this theme again primarily appeared in André's and Gustav's interviews. Gustav noted that he would describe the differentials in the separable ODE in a certain way if he is thinking "mechanical or applications." André described physicists as more willing to view differentials as real numbers than mathematicians, and discussed his own experiences of learning calculus as a student: there existed an "engineering way" in which $d x$ was a value smaller than scale, but also a "mathy way" which was based on traditional, epsilon-delta limits.

Theme (C).c: There is an awareness of nonstandard analysis. This theme differs from Themes N.4b and L.4b in that with this theme, the interview subject does not personally subscribe to the view of differentials as nonstandard infinitesimals. Rather, the interview subject mentioned that he or she was aware that it is possible to view differentials as some type of formally-defined infinitesimal, and that doing so would give the differentials a different flavor. In my dataset, this theme was used by Eugene and Francis, who, respectively, said toward the beginnings of their 
interviews "Although it is my understanding that, you know, in this theory of infinitesimals or whatnot that can be made precise, you can view it that way. I don't have that background, that machinery," and "I know a little bit about, with infinitesimals. I don't know a lot, probably not enough to hurt me, but, you know, that you think about these in a different, nonstandard way."

\section{Theme (C).d: The interview subject mentions that the act of teaching can alter one's}

views of differentials. This was the most common of "context" subthemes. Nearly every interview subject mentioned their teaching in some way during his or her interview, perhaps implying different "personal" and "instructor" aspects of one's concept image. The alteration of differential conceptualizations that comes from teaching took several forms in my dataset. I will mention and give examples of three.

First, it is possible that one might teach conceptualizations that are markedly different from one's personal ideas about differentials. This seems true with Bryan. As will be mentioned in his interview summary below, Bryan seemed to hold views that differentials were values that did not come from a limit. Nevertheless, when teaching, he presented limit-based differentials. The act of teaching caused him to use a conceptualization that fell outside his personal views. Second, sometimes instructors teach different conceptualizations to different types of students. Eugene gave two examples of this: he said that he would teach the $d x$ in a definite integral as a small width if he were teaching undergraduates, but as an indicator of the variable of integration if he were teaching more advanced students, and he would accept "multiply by $d t$ " from younger students without protest, but would want to "push a little bit further" if an older student said the same thing. Finally, it is also possible that one holds a complex view of differentials but teaches a less-complex view to his or her students. An example of this comes from Gustav's discussion of Leibniz notation. He understood that notation as a ratio of formal nonstandard infinitesimals, but "when I 
actually teach calculus, I tell them that this is pure notation ... The intuition this notation - again, come[s] from the fact that you can do this by using infinitesimals." Thus, he may present his students with an intuitive understanding of Robinson's infinitesimals, but will not present a formal definition of them.

Theme (C).e: The word "differential" may have multiple meanings. This theme represents any instances in which there was any uncertainty as to how the word "differential" is interpreted by the interview subject. Eugene, Francis, and Xavier initially defined the word "differential" using the aforementioned "Linear Approximation" idea $\left(\Delta y \approx d y=f^{\prime}(x) d x\right.$, where $\Delta x=d x$, but $\Delta y \neq d y$ ), rather than the two-letter symbol of the form " $d_{-}$". When this confusion happened, it was cleared up quickly, and once discussed, never affected the remainder of the interviews.

Theme (C).f: A single differential versus multiple differentials in one expression. This theme was rare, only used by Bryan and Eugene when discussing Leibniz derivative notation. Both of them mentioned that they could define a $d x$ if it appeared individually, but since they did not view " $\frac{d y}{d x}$ " as a ratio, the " $d x$ " in this notation had no meaning to them. Thus, a " $d x$ " may go from having meaning to not having meaning, depending on the notation used.

Theme (C).g: Differential conceptualizations depend on experience. This theme notes that one's conceptualizations might vary depending on how much experience with differentials he or she might have. Xavier discussed this theme by saying that a layman would view the word "differential" as a general small difference whereas a mathematician knows and uses a very precise and formal definition. Yanick discussed this theme in regards to his personal growth as a mathematician, noting that, as a student, he viewed differentials as variable indicators, but after earning multiple degrees, his concept image of differentials has evolved. 


\section{Theme (U): There is Uncertainty Surrounding Differentials}

This theme was in use whenever the interview subject expressed views that showed he or she was not fully confident in his or her opinions of differentials.

Theme (U).a: Acknowledgement of cognitive conflict. With this subtheme, the interview subject expressed awareness that he or she had said contradictory statements within the interview, and was not happy with the existence of personal cognitive conflict. This unhappiness might have been described directly by the interview subject, or might have been inferred by how strongly he or she seemed to want to resolve the cognitive conflict. An example of this is Francis viewing his "Linear Approximation" differentials (differentials of the form $d y=2 x d x$ ) as real numbers, when in all of his earlier discussions of differentials, he described them as unquantifiable: "but now I'm being cognizant of what I think about this, and what I originally said ... That these [at the $d y$ and $d x$ ] are not quantifiable. [Thinking] And I'd have to really think about rectifying this."

Another example is Yanick's awareness of his inconsistency of how confident he described algebra with differentials:

So I've been contradicting myself all over the place. On one end I'm saying that I can multiply by $d x$ like it's nothing, and on the other end I'm putting divide in air quotes cause I'm really worried about dividing something that's very small.

Theme (U).b: Some uncertainty in the interview subject's responses. In this theme, the interview subject might have made statements that showed that he or she was not completely confident in the explanations that were given. The fact that this was a very common theme implies the sheer vastness of possible conceptualizations of differentials and perhaps speaks to the need for this dissertation. Some examples of this lack of confidence include the interview subject admitting that he or she was unsure if the answers being given were correct, an example of this 
being Diane's multiple uses of the word "fuzzy" to describe her understanding of her phrase "infinitely small." Another example is the interview subject suggesting that the answer being given is but a smaller part of some "real" answer that is beyond the interview subject's understanding, as in Eugene saying, "I don't really have a formal understanding of $d x$, although I understand that concept can be made precise if you make appropriate definitions and that kind of thing."

\section{Algebra Themes: How Much Algebra can be Conducted with Differentials?}

Throughout my interviews, there was disagreement among the interview subjects as to how "real" any algebra performed with differentials might be. I have designated three subthemes to cover the possible responses on a "real/not real" continuum.

Theme (A): Any algebraic step performed with a differential is as real as the same step performed with a real or formal infinitesimal number. In this theme, statements that describe algebraic manipulations with differentials are given just as confidently as statements regarding algebraic manipulations with real numbers. Note that this does not mean that one who used this theme must think of differentials as real numbers, only that he or she had no qualms about multiplying or dividing differentials. Examples of this theme include using the statement "multiply by $d t$ " as a step in the solution process for an ODE, describing the definite integral's multiplication as " $f(x)$ times $d x$," and describing Leibniz derivative notation as " $d y$ divided by $d x$," as long as these statements were given without any hesitation or qualifications.

Theme (“A”): We cannot perform algebra with differentials, but we can perform "algebra" with differentials. The quotation marks are meant to denote an algebra in which manipulations with differentials are performed as if the differentials were real numbers or formal infinitesimals, but the interview subject does so while directly stating that such manipulations are not as genuine as they would be with real numbers. In my data, examples of this theme included 
the description by some interview subjects that the separation step in the ODE's solution was "like" or "as if" we are multiplying by $d t$, and the idea that, while one might teach such a separation as multiplication, one might also, to use Diane's words, "feel funny about writing it that way."

Theme (A): There is no algebra with differentials. In this theme, the interview subject believed that one cannot perform any algebra (or even "algebra") with differentials. The presence of this theme would be noted by an unambiguous statement that algebra is simply not allowed. To use the converses of the above examples, someone using this theme would not accept "multiplying by $d t$ " as a viable solution method for the ODE, would state that a definite integral should not be considered as $f(x)$ times $d x$, and would view Leibniz derivative notation as not a quotient of $d y$ divided by $d x$.

Note that it is possible for an interview subject to express multiple views on this continuum throughout his or her entire interview. Their "algebra" views might change depending upon the differential-containing expression and/or the context that they are discussing.

\section{Theme (P-L): The Existence of a "Pseudo-Limit"}

This theme was used whenever the interview subject described a limit process that was incorrect or differed significantly from the traditional epsilon-delta limit. The particular example of this found in my data was mentioned as a conceptualization in Artigue (1991) and Orton (1983).

Yanick used a definition for the differential $d x$ as $\lim _{\Delta x \rightarrow 0} \Delta x$, and Francis used $\lim _{n \rightarrow \infty} \frac{b-a}{n}$. If one assumes that any value of any differential must be nonzero, then defining differentials in these ways is a contradiction, as the most basic facts of limits and continuity dictate that $\lim _{\Delta x \rightarrow 0} \Delta x=$ $\lim _{n \rightarrow \infty} \frac{b-a}{n}=0$. If this were true, then every definite integral would have to be equal to 0 .

Note that simply stating one of these incorrect definitions is not enough to classify the relevant differential as coming from a pseudo-limit. When discussing graphical representations of 
Leibniz' derivative notation, Diane said 'I would basically say “as we take [ $\lim _{\Delta y \rightarrow 0} \Delta y$ and $\left.\lim _{\Delta x \rightarrow 0} \Delta x\right]$, this limit approaches this $d y$, or limit approaches this $d x$. But it never gets there, 'cause we're not actually making it zero, right?" She both notes that these limits, if left unchecked, should reach zero, and also qualifies her statement with "basically," implying that, to her, the differential's definition is more than this limit. The assignment of this theme to a differential occurred when these definitions of a differential were presented despite, and without attention to, the inherent contradictions present in these definitions.

\section{Themes (P) and (I): Differential Notation is Practical (or Impractical)}

Theme $(\mathrm{P})$ was used at any point when the interview subject mentioned the utility of differentials. This was usually presented as the idea that differential notations make ideas or solution methods more intuitive. Examples of this include the usefulness of Chain Rule notation $\frac{d y}{d t}=\frac{d y}{d x} \frac{d x}{d t}$ and the utility of solving differential equations by separation. Conversely, Theme (I) was only used by Bryan, and was meant to convey that he believed what the differential notation is trying to represent is confusing or unclear. His specific example discussed Leibniz notation and how he viewed the $d x$ in that notation as different from other $d x$ 's: "I think the world would have been fine if we would have called [the derivative] $y^{\prime}$ and never said that $\Delta x$ becomes a $d x . "$

\section{Summaries of Each Interview Subject}

This section will summarize both the main findings of each interview as well as provide the beginnings of an analysis of the personal concept definition of differentials that seems to be held by each interview subject. I qualify that last sentence with the words "beginnings" and "seems" because I recognize that providing a complete concept image of an object as complicated as the differential might not be possible after one interview. Nevertheless, I do believe that the 
information I obtained by using my detailed interview protocol allows for not only good approximations of personal concept images, but also overall trends and some interesting responses. André

André's themes were almost entirely non-limit-based, as can be seen by the collection of small boxes in the top half of his flowchart and the prevalence of red over green in Figure 11. The only times he described limit-based conceptualizations were during his discussions of the definite and double integral, stating that the differential in an integral served as an indicator that a limit process occurred: "the $d x$ represents the fact that it was a [Riemann] sum." Also, the idea of limitbased differentials in integration was almost always given with a discussion of the history of integration and notation. This is shown by the presence of Theme (C).a in the same column as most of his spreadsheet's green cells. Specifically, he called limit-based interpretations the "historical" way to interpret the differentials in those expressions, while stating that the "practical" and preferred interpretation was of these differentials as variable indicators. Thus, I might suggest that his limit-based themes seemed to have been stated as to give an idea of completeness instead of being conceptualizations he actually believed and held.

Interview subjects having multiple conceptualizations was a common occurrence throughout the data, and André was an example of this. Specifically, he seemed to have a pretty even split between differentials as well-defined numbers and differentials as variable indicators, shown in his flowchart as a balance between themes on the right and left sides, respectively, and in his spreadsheet as a pretty even split between dark red cells and light red cells, respectively. When discussing his phrase "infinitesimally small" and Leibniz derivative notation at the beginning of his interview, he described differentials the following way "If they represent actual variations, actual quantities, then they're very small compared to - not so much to $x$, but more 


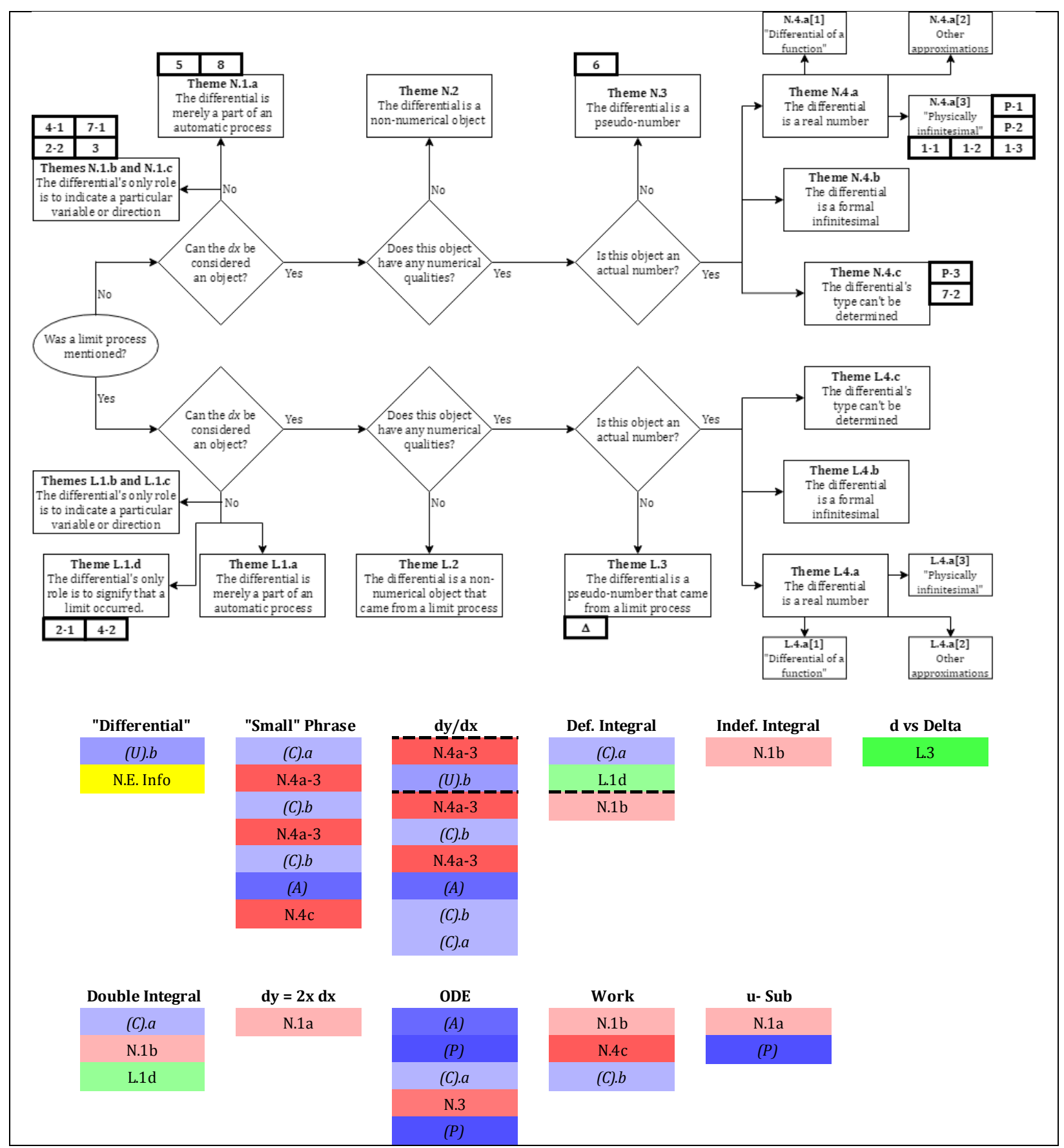

Figure 11: André's flowchart and spreadsheet

like, see [gestures at part of a figure he drew] to the scales at which $x$ or $y$ varies significantly," which seems similar to Courant and John's (1965) "physically infinitesimal." This "physically infinitesimal" description was also used when discussing the differentials in the separable ODE and the "Work" problem. However, when discussing integration and ideas like differentials of 
functions and integration by substitution, André's differentials were variable indicators and parts of automatic processes: "it's a necessary way to indicate what is the variable with respect to which you are, you are doing the integration or the antidifferentiation."

Intermingled with André's multiple conceptualizations was the idea that different contexts can affect how one might conceptualize differentials. This idea was briefly explored during my pilot study, which involved interviewing two mathematicians and a physicist, but André's interview was notable for how often context was discussed, as shown by the amount of "(C)" themes in the spreadsheet in Figure 11. Not only did André mention some differences in how he felt mathematicians interpreted differentials versus physicists or engineers (Theme (C).b), but he also claimed different interpretations could exist based on the historical development of notation (Theme (C).a). Specific examples of these include

So just, just imagine $d x$ is a really, really, small number, you know, very, very small change in $x$, which is smaller than anything, anything else $x$ might do. And then we do these things ... you can do simplifications, you can do algebra ...that was the "engineering way" of teaching Calculus. And then the "mathy way" was the epsilon-delta ... "the practical role is that it [the $d x$ in a definite integral] just tells you what you're integrating with respect to. And, as an historical or kind of symbolic thing here, it represents that it comes from the $\Delta x$ and the $\Delta y$ in a Riemann Sum."

\section{Bryan}

Bryan's dominant view of differentials was one of differentials as real numbers, as can be seen by the concentration of themes in the top right of his flowchart and by the amount of dark red cells in his spreadsheet (Figure 12). I would guess that at least a part of this view came from his 
non-academic work, which involves mathematical modeling. Many times during his interview, he mentioned hypothetical situations such as receiving values from a colleague with which he would need to calculate a derivative, or needing to approximate an integral within a certain level of tolerance.

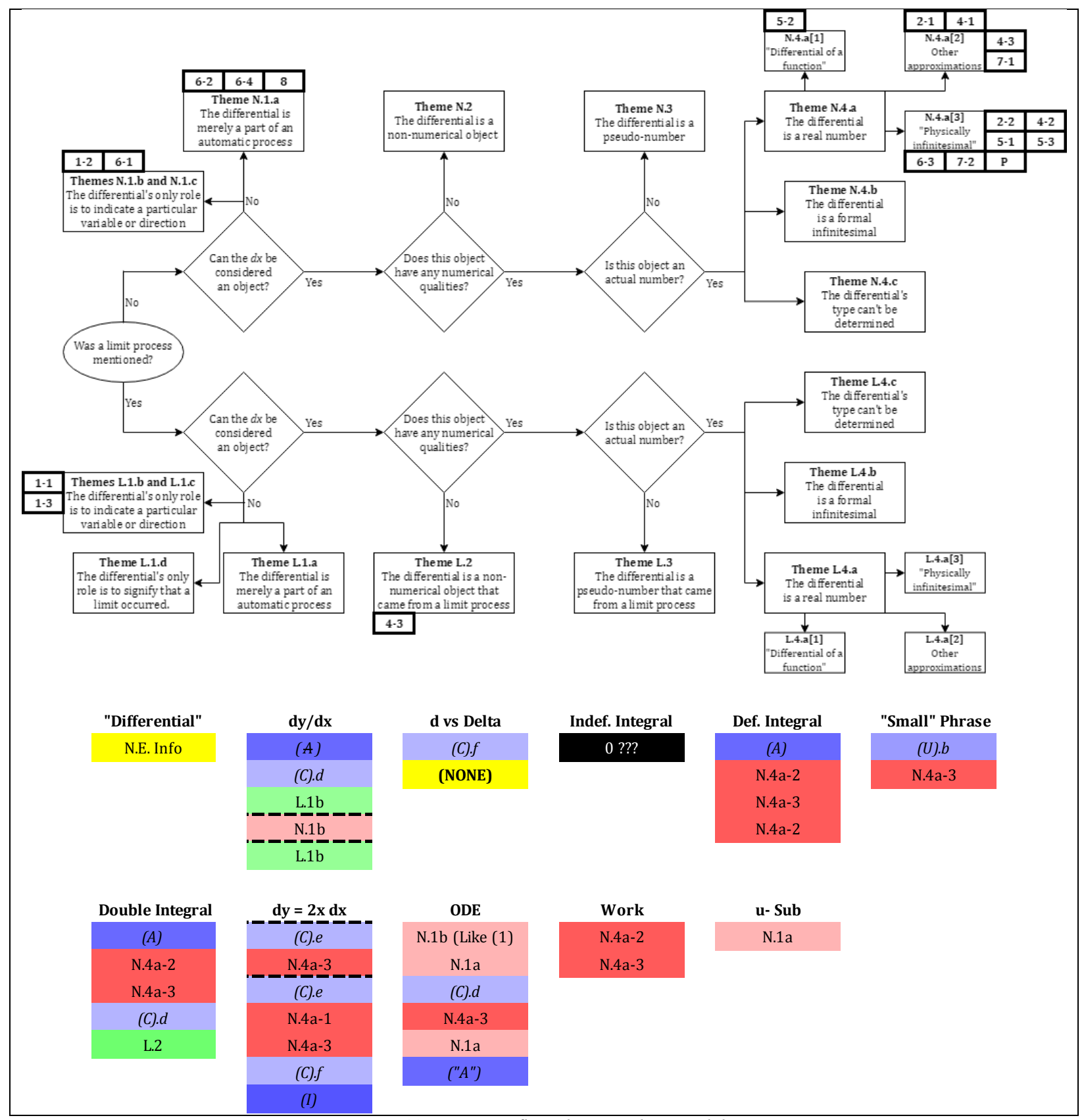

Figure 12: Bryan's flowchart and spreadsheet 
Thus, for Bryan, the differentials in definite integrals, double integrals, and the expression $d y=2 x d x$ were real numbers. In fact, when asked about phrases like "infinitely small," he said not only that he did not like to use them because they were too difficult to make precise, but also "I don't think of [the definite integral's $d x$ ] as infinitely small. It's always ... a finite number." Sometimes he interpreted this number in the sense of Courant and John's (1965) "physically infinitesimal" in which the differential should be smaller than the relevant scale of the physical situation:

If we're talking about astronomical stuff, you know, the change in $x$ being small could mean 100,000 miles, but when you're talking about molecules, you know, the change in $x$ can not be 100,000 miles, it's one Ångström. So "relative" is important, but for this idea that the change in $x$ will help you determine the change in $y$, the change in $x$ has to be relatively small

Sometimes this real number was simply a value not dependent on a particular scale, but instead simply small enough to give him the level of approximation he needs:

I should be able to come up with a way to come up with an approximation that's better and better. You know, if somebody says "I want it to $10^{-6 "}$ I can do it, or if they want it $10^{-12}$ I can do it ... the $\Delta x$ would be $10^{-4}$ or something.

There were three main instances in which Bryan did not use real-number-based conceptualizations for differentials. First, he did not have any conceptualization for the $d x$ in the indefinite integral: "To me, [the indefinite integral's notation] is a straightforward way of coming up with the antiderivative of $g$. And [the $d x$ ] could almost mean nothing." The idea that a differential has no meaning is represented by black "Theme $\# 0$ " cells in the spreadsheets. Second, he viewed the solution of the separable ODE and the evaluation of the integration by substitution 
expression as automatic processes. Consequently, the differentials in the ODE and "substitution" integral were merely parts of this automatic process and not necessarily real numbers. Finally, Bryan occasionally used "limit-based" themes, but he only did so when he was discussing his teaching, as shown by the inclusion of the "teaching context" Theme (C).d in the same columns as the only green cells in Figure 12. He mentioned the idea of secant lines approaching a tangent line when discussing Leibniz derivative notation and shrinking $\Delta x$ and $\Delta y$ when defining a double integral, but he prefaced both of those ideas with phrases like "If I were speaking to a student." I would say that, while his personal concept image is mostly one of differentials as real numbers without limits, Bryan also has elements of a limit-based "teaching" concept image that he can use when needed. This is another, and different, example of context-based differentials than the ones found in André's interview.

\section{Christopher}

Compared to André and Bryan, Christopher used limit-based themes much more often, as his is the first flowchart to have a lot of themes on the bottom half and a spreadsheet containing a lot of green (Figure 13). Indeed, the differentials in his definite, indefinite, and double integrals would best be described as limit-based pseudo-numbers. I call them "pseudo-numbers" because he described and drew the width of the Riemann sum rectangles under a curve as " $d x$ " and later said " $f(x)$ times $d x$ " when describing the integral These imply that his $d x$ can be multiplied and used to measure a width, which I would describe as "numerical characteristics." However, unlike André's and Bryan's differentials that were specifically defined as real numbers, albeit smaller than a particular scale, Christopher did not quantify his integral's differentials, and only described them by the more vague "Really small and getting smaller. As a limit."

Other limit-based themes arose with the questions not directly addressing differentials in 


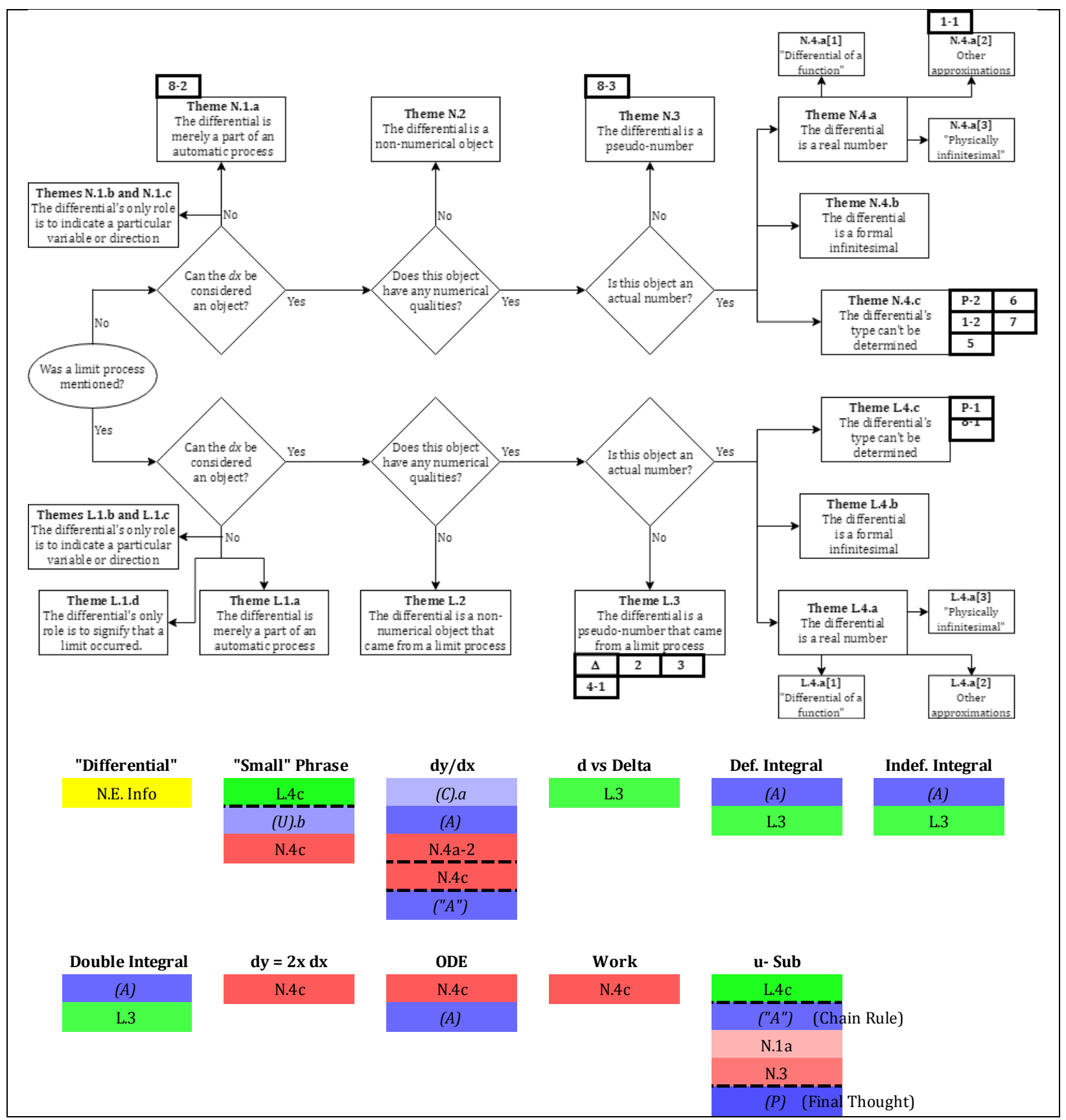

Figure 13: Christopher's flowchart and spreadsheet

expressions. When discussing his initial phrase that differentials were "teensy-weensy" changes in a variable, he later clarified this definition with "Differentials kind of represent the limiting form of linear approximations." When discussing the difference between $\Delta x$ and $d x$, he stated "When 
you write $d x$, you're in some sense taking a limit; there's some sort of limit involved ... Yeah, there's some sort of limiting process involved when you use a ' $d$ '.'

Differentials that were measurable quantities and not limit-based occurred during his discussion of the "context" problems. Even though he described the differentials in the definite integral as limit-based pseudo-numbers, when discussing the definite integral in the "Work" problem, he ascribed to its differential a definable length with no mention of limits: "So $d x$ would be a small change in the position of the mass going up the side of the building," and "I usually like to show them that the work is the same if you imagine lifting only the mass by itself, and then chopping up the cable into little bits, and raising each little bit by itself." Also, when describing how he tells his students how to model a differential equation, Christopher stated "I usually tell my class 'If you want to model a physical situation, get a differential equation out of it, you gotta ask "What happens over the next $d t ?$ ?"," implying that the $d t$ is a measurable value of time not described in terms of a limit. This seeming discrepancy between limit-based and non-limit-based themes might have come about from imprecise or incomplete questioning by me, but I think that instead, it is an example of a concept image that contains two aspects that are evoked at different times: a "theoretical" aspect that is evoked when discussing theory and generic expressions, and a "practical" aspect that is evoked when discussing expressions within a physical context.

\section{Diane}

The summary for Diane's interview is perhaps the most streamlined: she viewed almost every differential with a limit-based theme, as shown by the amount of themes in the lower half of her flowchart and the amount of green in Figure 14. In fact, there were two interview expressions, the separable ODE and the expression $d y=2 x d x$, for which she was the only interview subject to invoke limit-based themes for the differentials contained in those expressions. There were a few 


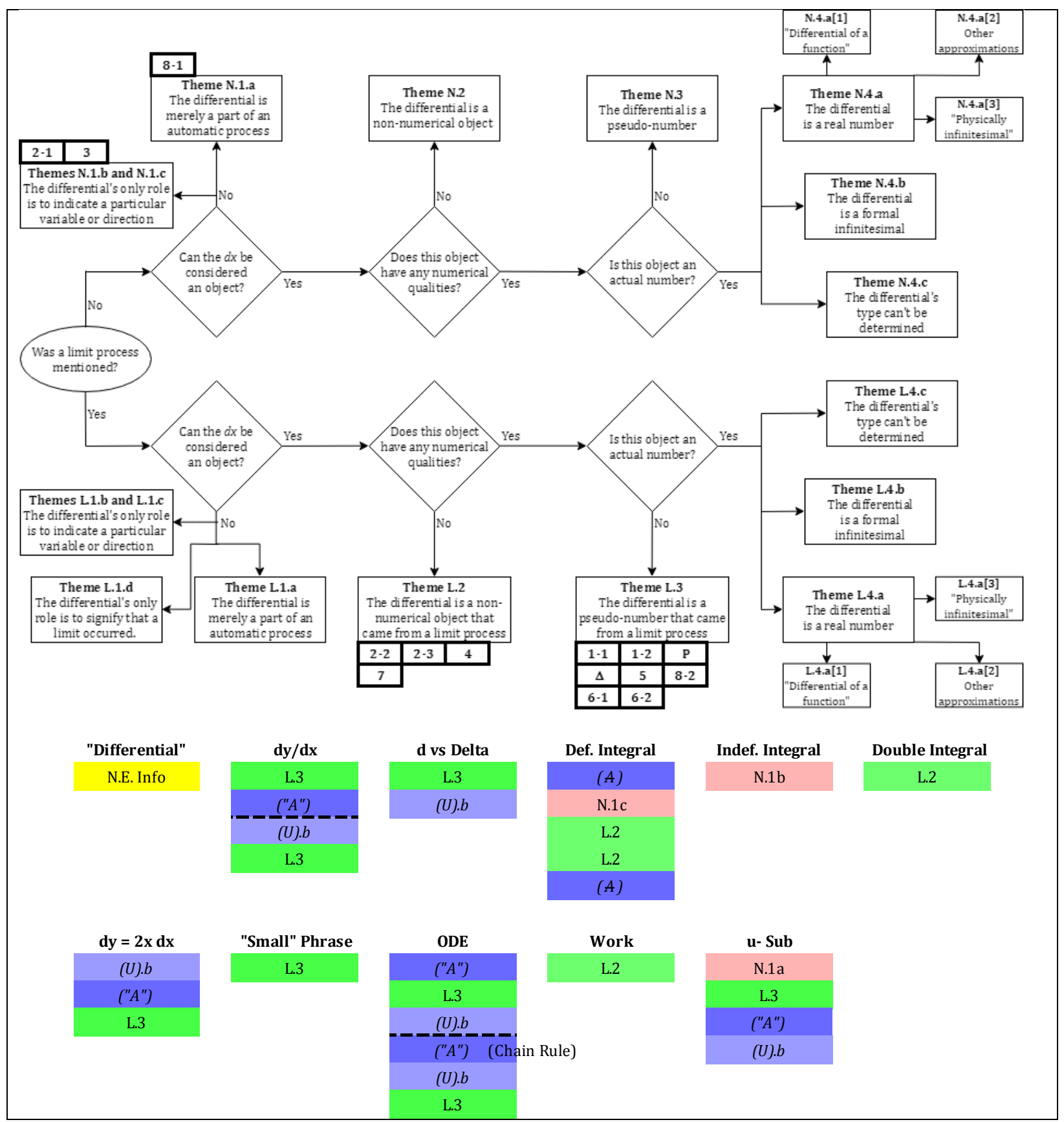

Figure 14: Diane's flowchart and spreadsheet

other differentials that she could view as variable indicators, but for most of those differentials, she held a second view that they also could arise from limit-based themes. The differential in the indefinite integral was the only one that she described as purely a variable indicator without any limit basis. 
Diane seemed to split her limit-based differentials into two groups, depending on her views of how similar they were to well-defined numbers. The first group was differentials as limit-based pseudo numbers (Theme L.3). For these differentials, Diane was willing to say, albeit uncomfortably, that one can tend to "treat them as actual quantities that we can divide by and move around." For example, when solving an ODE, she said that it is acceptable to "multiply by $d t$ " in order to separate the variables as long as it is understood that the $d t$ does not represent a real number: "I keep saying things like "It's an infinitely small quantity; it's not really a number, but we think about it that way." The other group described differentials as reified limit processes (Theme L.2): the differential was an object that has equal status with any other symbol in an expression, but has no numerical qualities. For example, Diane described the differential in a definite integral as an object that conveys information about variable or direction, but also said "I don't ever really think about it as being $f(x, y)$ times $d x$ itself," which would not qualify this differential as a pseudo-number. Thus, it would be too simple to define Diane's concept image merely as consisting of "limit-based" differentials, since, for her, some of these differentials have numerical characteristics and some do not, creating nuance to her concept image, as shown by the two slightly-differing shades of green throughout her spreadsheet.

However, it is perhaps worth noting that Diane was the interview subject who was assigned the greatest number of "uncertainty" themes (Theme (U).b in her spreadsheet). Examples of this uncertainty included her inability to describe fully her term "infinitely small," not being certain whether it was proper to multiply, divide, or cancel differentials, and stating that textbooks "come up with some funny, hand-wavy thing - way to explain what they're doing there" when they are discussing differential-based ideas. Thus, part of her concept image is one in which differentials are somewhat nebulously-defined. 


\section{Eugene}

The most striking thing about Eugene's interview were his two views of similar terms. When discussing the phrase he uses for an idea of "smallness," he gave different definitions for the terms "infinitesimally small" and "infinitesimal", shown by the different colors in the "Phrase" column of his spreadsheet (Figure 15). He described "infinitesimally small" as "something that's

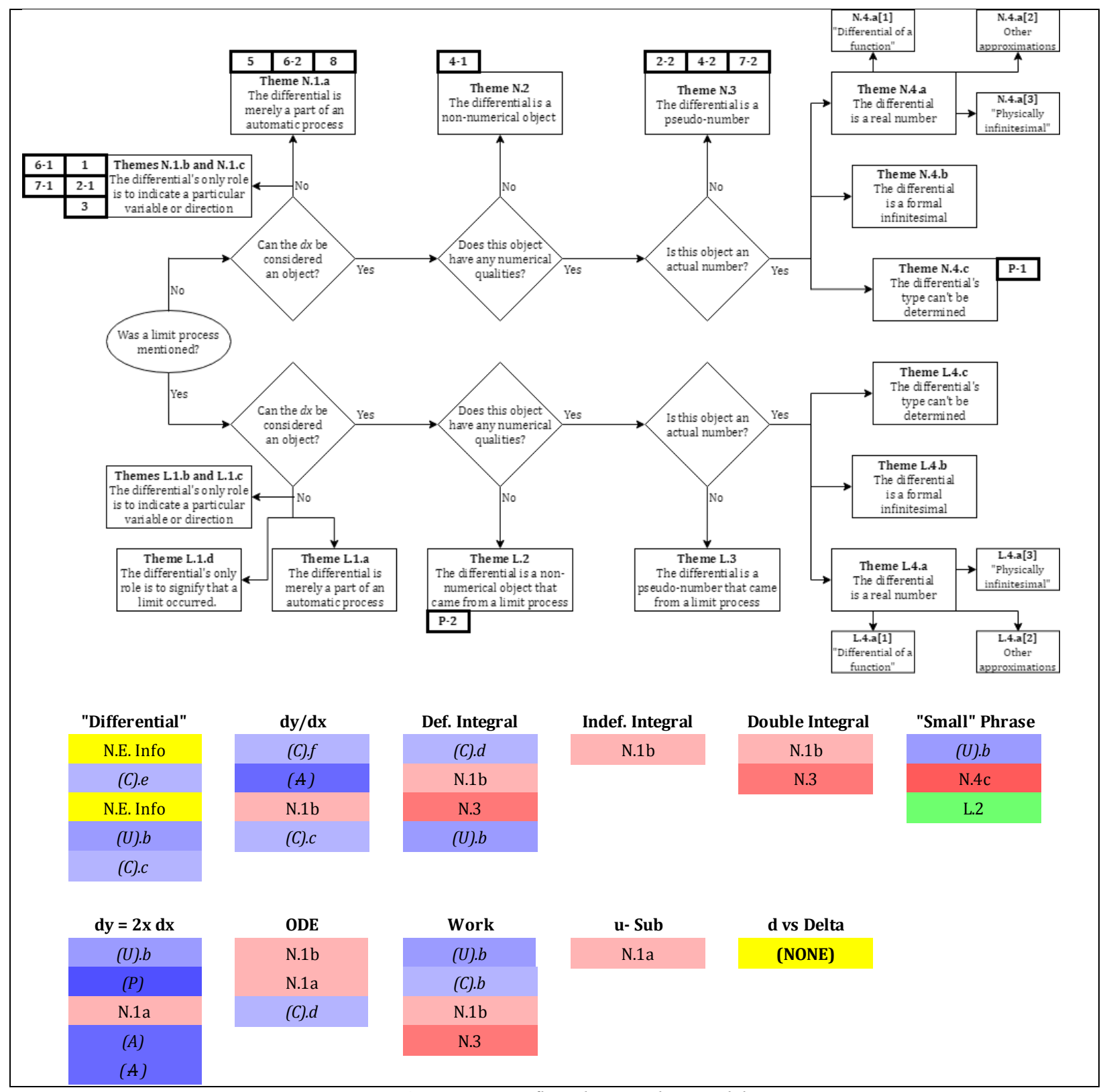

Figure 15: Eugene's flowchart and spreadsheet 
sort of a picture of the limiting process at a very advanced stage," while "infinitesimal" was defined "somehow after it's all done and packaged in a [makes a "container" gesture], you know." It seems to me that there was a difference between the terms "very advanced stage" and "done," and that the choice of these words causes his definitions to be different. His "infinitesimal," which was used when the limit process is done, seems to describe the reification of the limit process into an object, an idea perhaps reinforced by his "packaging" metaphor and "container" gesture. But if one interprets "advanced stage" as a limit that is still in the process of shrinking, then his “infinitesimally small” might represent a measurable quantity.

It might seem that, with all of this talk about shrinking, Eugene's concept image tends towards limits in some form when discussing the theory of differentials. In practice, however, Eugene most often referred to differentials in simpler ways that did not involve limits, as shown by the placement of themes in his flowchart and lack of green in his spreadsheet. Common responses were differentials as variable indicators (Theme N.1b) in Leibniz derivative notation and integrals, or differentials as parts of automatic processes, such in integration by substitution: My first cut at [the integration by substitution] is that this is just an algebraic device which facilitates ... what would formally be to re-write, to put the integrand in terms of the result of a differentiation of a composition of functions. So I see it as kind of a time-saving informality.

Thus, the $d u$ and $d t$ in the substitution $d u=\frac{1}{2 \sqrt{t}} d t$ are written without any thought given to their conceptualization. Other responses by Eugene painted some differentials as pseudo-numbers (Theme N.3). Like Christopher, he also wrote " $d x$ " as the width of the Riemann sum rectangles for his definite integrals, implying that the differentials in definite and double integrals had a "measurable" quality, even if they were not thought of as an actual value. 


\section{Francis}

Francis' concept image began to take shape as one in which there were initially two main conceptualizations of differentials depending on the expression in which those differentials resided. This can be seen most easily in the first row of his spreadsheet (Figure 16), in which his “integral" columns contained green, limit-based themes, but all other columns contained red, nonlimit-based themes. One of these main conceptualizations was that of a differential as a variable “integral" columns contained green, limit-based themes, but all other columns contained red, nonlimit-based themes. One of these main conceptualizations was that of a differential as a variable "integral" columns contained green, limit-based themes, but all other columns contained red, nonlimit-based themes. One of these main conceptualizations was that of a differential as a variable indicator. This could be inferred in that, while he said that the notation $\frac{d y}{d x}$ was one symbol, he said "I assume $y$ is a function of $x$ " when first viewing it, suggesting that this symbol identified the dependent and independent variables by their placement in that symbol. Thus, the purpose of the $d y$ and the $d x$ were to indicate the dependence of the variables. Similarly, when seeing the indefinite integral symbol, Francis described it as "the antiderivative of $g(x)$ with respect to $x$," suggesting that the $d x$ indicated the variable of integration. The second main conceptualization was one of the differential as a limit-based pseudo-number (Theme L.3), found in his views of the definite and double integrals. This can be inferred because, while he described the word "differential" as "an infinitesimal change" and this change as more of a concept than something that can be quantified, he continually described the differentials in his definite and double integrals as "widths" that come from limits and can be multiplied, suggesting that even if differentials cannot be quantified, they at least have numerical characteristics.

Later in the interview, however, his complex image became more complex, as he became 


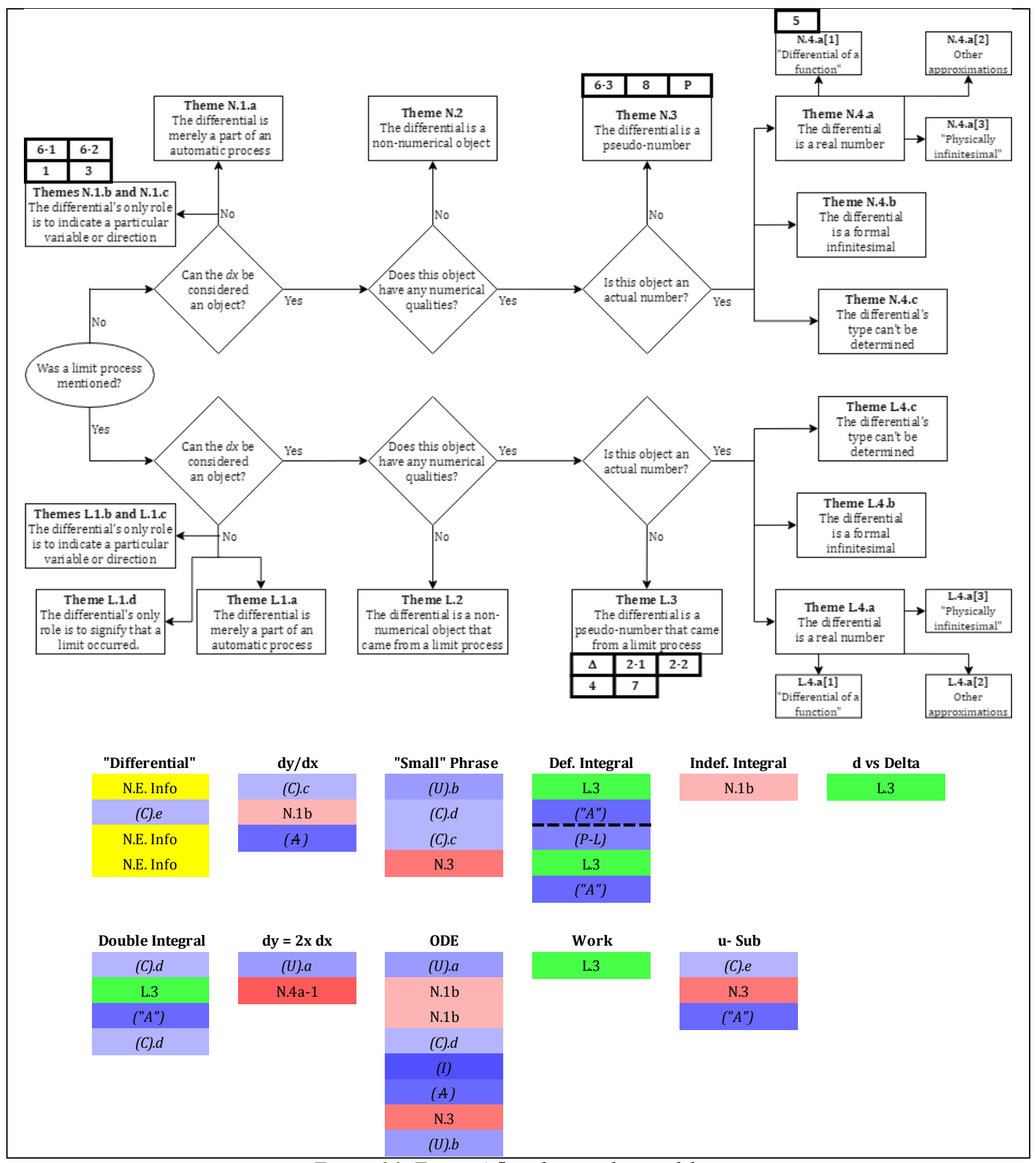

Figure 16: Francis' flowchart and spreadsheet

the first interview subject to admit to having cognitive conflict over conflicting responses. This is shown in the spreadsheets by Theme (U).a. His cognitive conflict began when describing the differentials found in the expression $d y=2 x d x$. He described these differentials by what I called 
the "Linear Approximation" idea in my literature review: $\Delta y \approx d y=f^{\prime}(x) d x$, where $\Delta x=d x$, but $\Delta y \neq d y$. Since $\Delta x$ is a real number in these expressions, $d x$ would also be a real number, and so, by extension, would $d y$. After describing what were, to him, the now-quantifiable differentials in the expression $d y=2 x d x$ and after I confirmed that these differentials were quantifiable, he noted, "but now I'm being cognizant of what I think about this, and what I originally said, no. That these [the $d y$ and $d x$ ] are not quantifiable. [Pause] And I'd have to really think about rectifying this." A second instance of cognitive conflict occurred discussing the separable ODE $\frac{d \tau}{d t}=-k t$ when he considered both his previously-stated idea that $\frac{d y}{d x}$ was one symbol and not a quotient with his idea that you can separate the $d \tau$ and $d t$ during the solution of the ODE. Although he later solved the same ODE by integration, thus showing that this separation was not mandatory, he did mention that he was aware of this discrepancy and that textbooks do not explain this discrepancy very well.

\section{Gustav}

Gustav was the first interview subject, and the only interview subject within the first seven interviews, to discuss the idea of differentials being formal infinitesimals as defined in nonstandard analysis (Theme N.4b). He specifically mentioned Abraham Robinson multiple times, and when using the word "infinitesimal" to describe a differential, stated his definition of "infinitesimal" by saying: "Well, I can give you the definition: that's smaller than any $\frac{1}{n}$ and greater than zero. That's kind of [a] positive infinitesimal." The existence of hyperreal infinitesimal values greater than zero yet smaller than any real number is central to nonstandard analysis.

Yet, I believe that it is noteworthy that his only uses of the idea of "formal, nonstandard infinitesimal" occurred during the discussion of the three more "theoretical" questions (What does the word "differential" mean to you? What is the difference between $d x$ and $\Delta x$ ? What do you 
mean by "infinitesimal"?). This might be an example of what I will call an "unpacking" of his concept image. When first presented with an idea, he defaulted to the immediate recall of specific, nonstandard-infinitesimal-based theoretical definitions that he had memorized and could recite, but when asked to think about the idea some more, he drew upon other elements of his concept

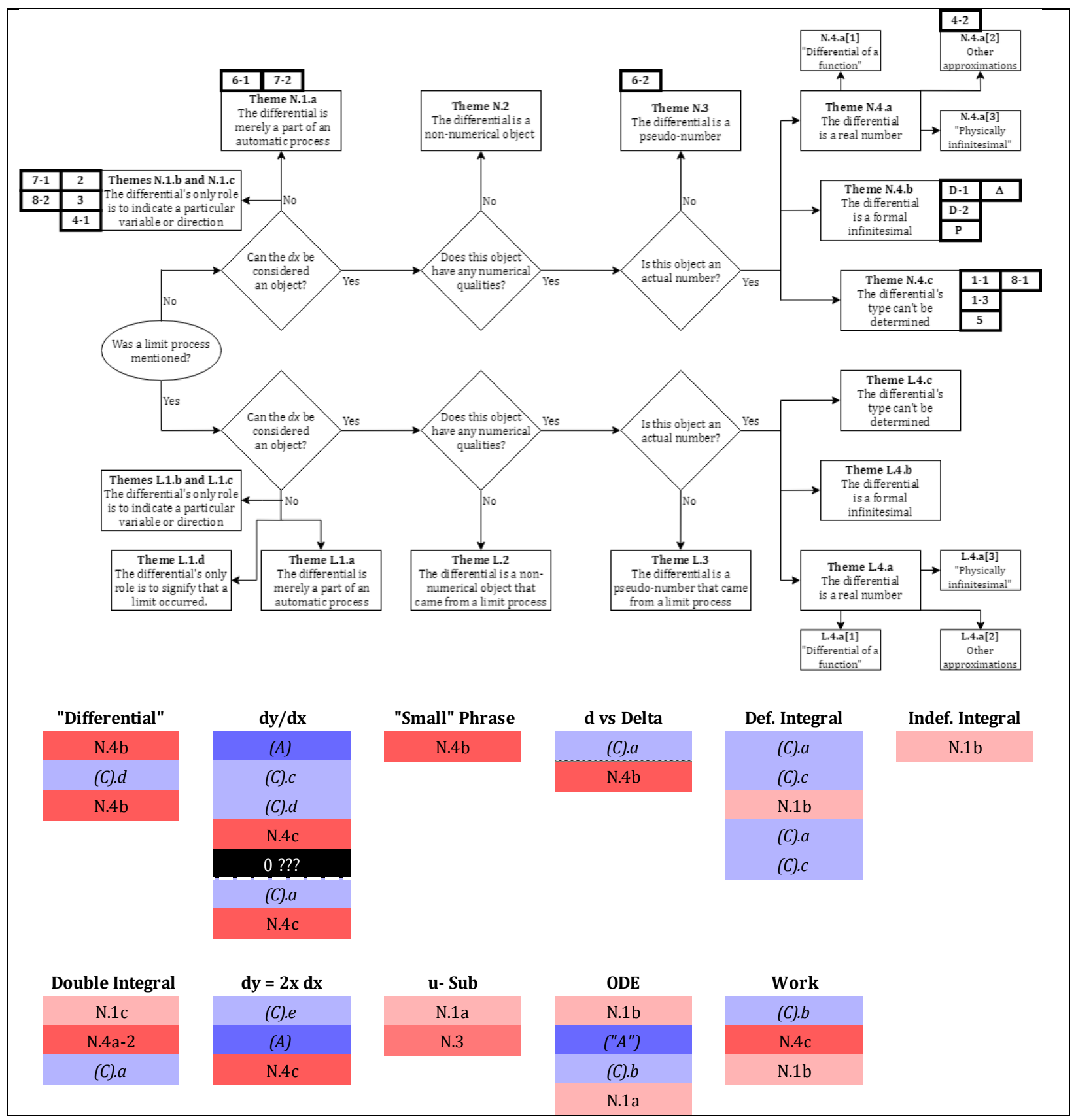

Figure 17: Gustav's flowchart and spreadsheet 
image to broaden this initial recall. This can be seen in his spreadsheet, in which, after the last "theoretical" question, not only were there no more occurrences of Theme N.4b, but there was also in increase in theme diversity. A similar "unpacking" idea might explain why all other interview subjects answered the initial "differential" question with vague, poorly-defined notions of "small" that did not contain enough information to justify the assignment of a theme. The prominent characteristic of the generic differential might be "it is small," and this characteristic is the one that might be the most easily recalled. Then, when given specific expressions and asked to qualify one's thinking, interview subjects want to move beyond one simple characteristic and provide more details from their concept images. Still, it is notable that Gustav's initial "small" response distinctly mentioned formal infinitesimals when no other initial responses to this point did.

Gustav went on many tangents during his interview, including his opinions on calculus instructors' limited knowledge of differential-based calculus, historians' comparisons of Leibniz and Newton with Robinson, and his views of what should be taught in first-year versus later-year calculus courses. When addressing the expressions from my protocol, Gustav used the word "limit" only once, and that mention of "limit" was used to discuss a view that, to him, is rarely used:

There are several ways of doing it ... when you take the integral as approximation ...you split this into equal pieces - as a limit, you obtain the integral. However, most of the time, you don't think about this when you are truly trying to solve this. You just know that this [at $W=\int_{a}^{b} F d x$, a formula he earlier wrote and discussed] is a formula with 0,50 , and put it here [at the "Work" integral]. Similar to André, most of Gustav's themes were the non-limit-based themes of "variable indicator" or "automatic process" on one side, and "actual number" on the other, but unlike André, the 
differences between these two ideas did not split as neatly along a "differentiation/integration" divide. Rather, there were "variable indicator" and "automatic process" themes in almost every expression. Like André's interview, there was a lot of contextual discussion, including both historical contexts (shown by Theme (C).a) as well as physics contexts (Theme (C).b), suggesting that perhaps Gustav primarily uses differentials in simple ways like variable indicators or as parts of automatic processes, but he also understands and can accept historical or physical reasons why a differential might be perceived as a well-defined value, as long as those historical and physical reasons do not involve limits.

\section{Xavier}

The last three interviews were conducted after the completion and analysis of the first seven. However, each of the last three interviews contained themes and ideas not found or not fully-explored in the first seven. For example, early in his interview, Xavier suggested the existence of a hitherto unused context: that of "layman versus professional" (Theme (C).g). Specifically, he noted that the word "differential" means the value $f(x+\Delta x)-f(x)$ "at a layman level", whereas "mathematically" and "rigorously," the word differential has the "very precise" definition of $d y=f^{\prime}\left(x_{0}\right) d x$. It is noteworthy that he gave the precise definition of "differential" as $d y=f^{\prime}\left(x_{0}\right) d x$, yet called the " $d x$ " in that expression an independent variable, equal in status to the other independent variable:

To begin with, we start with $d y$ to be defined as $f^{\prime}\left(x_{0}\right)$ times $d x$. And to be very accurate: $f^{\prime}\left(x_{0}\right)$ should be considered one variable, $d x$ another variable independent variable. So altogether we have two independent variables: $x_{0}$ and $d x$, and when you multiply them together, you get $d$ 
Thus, it appeared that the status of "differential" was not automatically conferred by the symbolic form $d[]$, since " $d x$ " is treated the same as the $x_{0}$, while " $d y$ " is afforded "differential" status.

In addition, the idea of " $d x$ is an independent variable" was not consistent throughout his

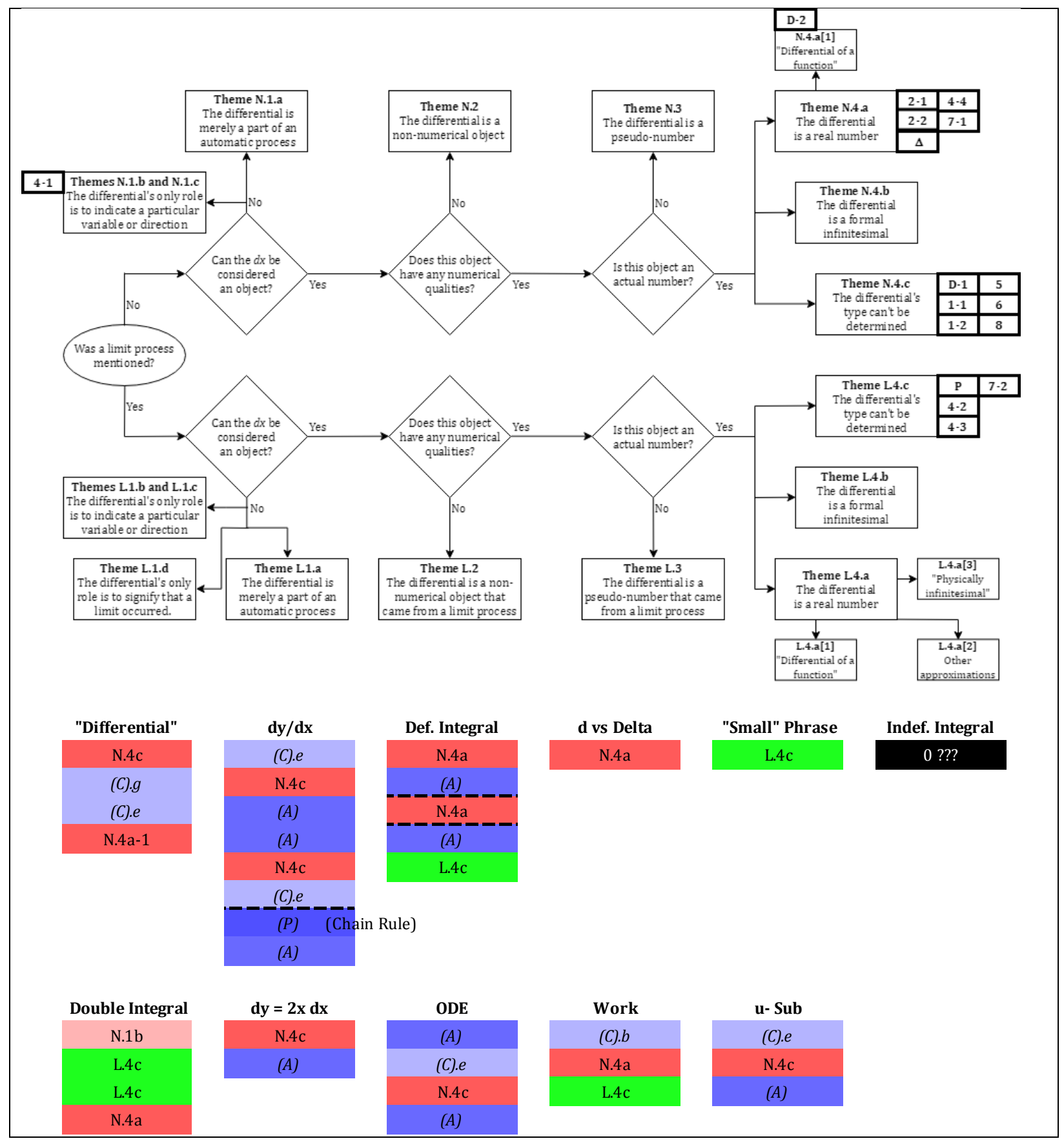

Figure 18: Xavier's flowchart and spreadsheet 
entire interview. When discussing Leibniz derivative notation, he did refer to the above definition $d y=f^{\prime}\left(x_{0}\right) d x$, stating that as long as $d x$ did not equal zero, division of it in order to obtain $\frac{d y}{d x}=$ $f^{\prime}\left(x_{0}\right)$ was fine. But when discussing the $d x$ in a definite integral, Xavier did not refer to that definition. He initially defined that $d x$ as the difference of two partition points $x_{j}-x_{j-1}$, and thus a real number, but after giving that definition, described the product $f\left(x_{j}^{*}\right)\left(x_{j}-x_{j-1}\right)$ as infinitesimal. This seemed to blur the distinction between $x_{j}-x_{j-1}$, being a "real number" or "infinitesimal," until he gave his definition of "infinitesimal" as "the process in which you take a

quantity to the limit zero. In this case, $x_{j}-x_{j-1}$." This recent addition of the idea of limit can be seen in his spreadsheet, where the first green-colored limit-based theme occurred at the end of his discussion of the definite integral, and then reoccurred sporadically throughout the rest of his interview whenever he discussed integration.

Thus, as had occurred with many of the other interview subjects, Xavier's concept image seemed to have multiple strong themes, their "strength" shown in the flowchart by the multiple squares attached to them. For him, his initial definition of the word "differential" involved a formula that referred to $d y$ and not $d x$ and drove the ideas of Leibniz derivative notation and the substitutions used in integration by substitution. But the $d x$ 's found in integration were ones that were well-defined, measurable, infinitesimal differences that resulted from a limit.

\section{Yanick}

Yanick's concept image was one in which differentials are predominantly pseudo-numbers (Themes N.3 and L.3 on the flowchart and in his spreadsheet). I characterize most of his differentials as pseudo-numbers based on two recurring statements. On the one hand, he described the idea of "infinitely small change" as a "theoretical construct" that is "not necessarily physical." On the other hand, he said many times that he has no issue with multiplying or dividing by a $d x$ if 
needed. Entities that can be treated as numbers without being quantifiable is how I define pseudonumbers in this dissertation. However, unlike the views of some earlier interview subjects, who almost always described their pseudo-numbers as arising from limit processes, Yanick's

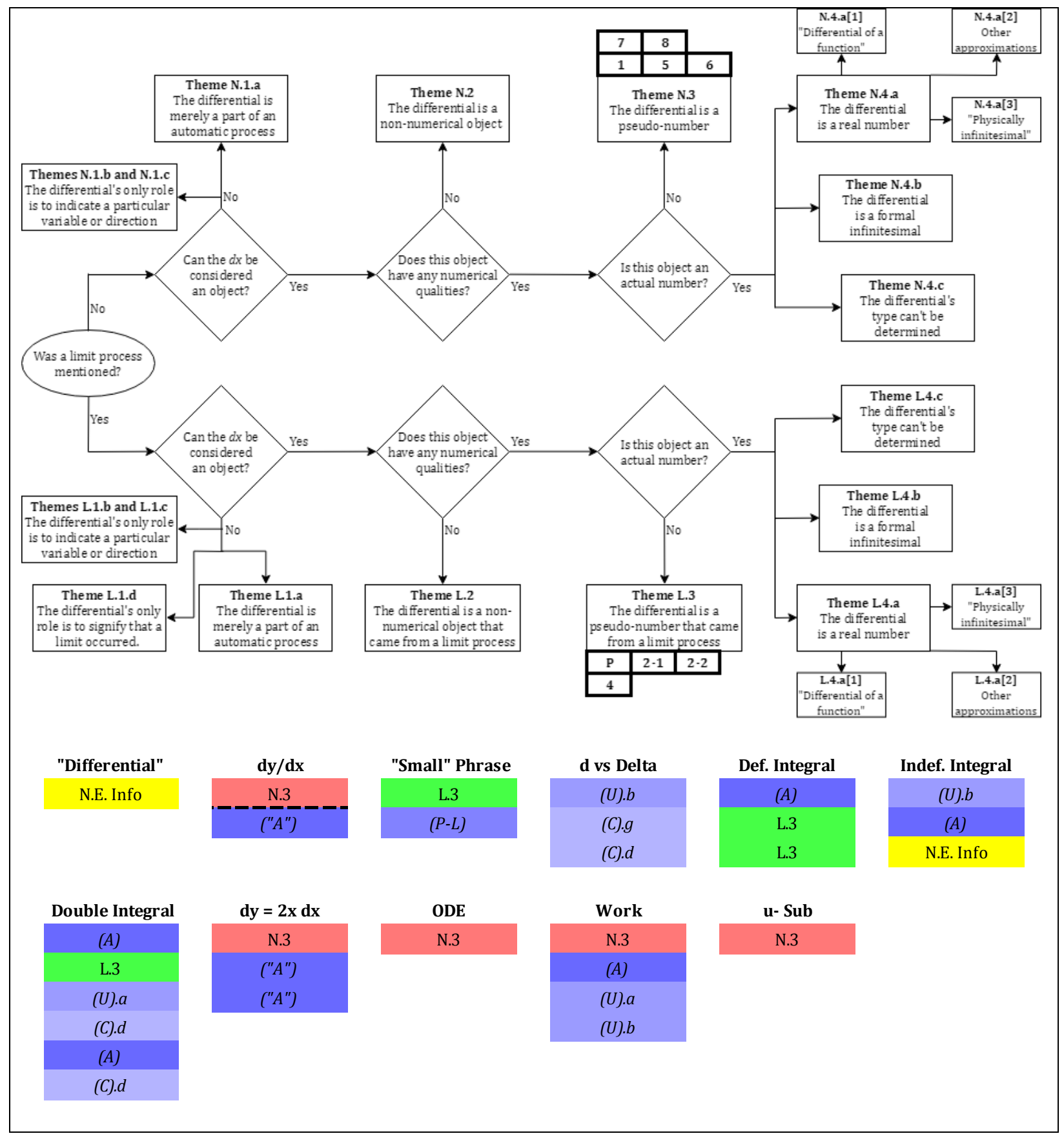

Figure 19: Yanick's flowchart and spreadsheet 
pseudo-numbers were almost evenly split between pseudo-numbers that arose from limits and pseudo-numbers that did not. Like many of the interview subjects before him, Yanick conceptualized the differentials in definite and double integrals as limit-based, while many of his other differentials, including those in the "context problems," were not-limit based.

There were three responses that seem worthy of mention. First, Yanick noted that he viewed some differentials as comparisons to changes rather than the changes themselves. When discussing Leibniz derivative notation, he said:

But I also want to be careful here. It's a comparison instead of a quotient to me. I'm comparing two quantities. Because you could easily as well, if $f(x)$ is an inverse function or $y$ is an inverse function, you could compare $d x$ and $d y$, and when discussing $d y=2 x d x$, he noted:

So basically this is a comparison to small changes, right? ... And then you gotta be VERY careful thinking about thinking about comparisons instead of thinking about divides or multiplies or what have you. Especially with chain rule or multivariable, and so forth. Yeah, in some sense that gives you intuition when I say "divides" (big air quotes). In another sense, we gotta be very clear about what comparisons are versus actual calculations.

It appears that Yanick views these particular differentials as comparisons in order to rationalize the idea that dividing by differentials is not permitted even though it may appear permitted in certain contexts.

Second, Yanick also referenced the new "layman versus professional" context (Theme (C).g). However, unlike Xavier's use of this context, where he seemed to compare a generic layman and generic professional, Yanick described this context as it pertained to his own 
mathematical development: when he was an undergraduate student, his only view of differentials was that of a variable indicator, but as he matured in his mathematics studies, his views of differentials became more complex. Yanick said that he tries to teach his students that differentials are more than variable indicators, so that they do not have to go through the same conceptual metamorphosis that he did. Finally, Yanick was the second interview subject to admit to cognitive conflict due to an internal contradiction (Theme (U).a in his spreadsheet. After initially saying that the $d y$ and $d x$ in a double integral have no graphical representation, he later stated 'No, I'm contradicting myself but that's alright. I do have certain times where I say if I zoomed in an infinite amount of times there would be a little change in $x$ and a little change in $y$," implying a graphical representation. But after saying this, he remarked that "There's two things swimming around here. One is trying to get students to buy into this stuff. And two is being true to what I feel like it means." Those last two statements painted a picture of a mathematician who possesses a personal concept image that revolves around unquantifiable pseudo-numbers, yet who also possesses a "teaching" concept image that he uses in an effort to make differentials more understandable to his students.

\section{Zaphod}

Zaphod's interview contained some similar results to Gustav's. I categorized no themes from Zaphod's interview as limit-based, so there are no themes at the bottom of Zaphod's flowchart and there are no green cells in Zaphod's spreadsheet. Also, like Gustav, Zaphod also mentioned formal infinitesimals as defined in Robinson's nonstandard analysis (1961). However, these interviews were only similar to one another for these particular characteristics at the surface level; many differences were found during further analysis. 
First, while Gustav mentioned nonstandard infinitesimals only during what I call the three "theoretical" questions and not during discussions of the actual differentials, Zaphod was the first interview subject to state that he thought of specific differentials as nonstandard infinitesimals:

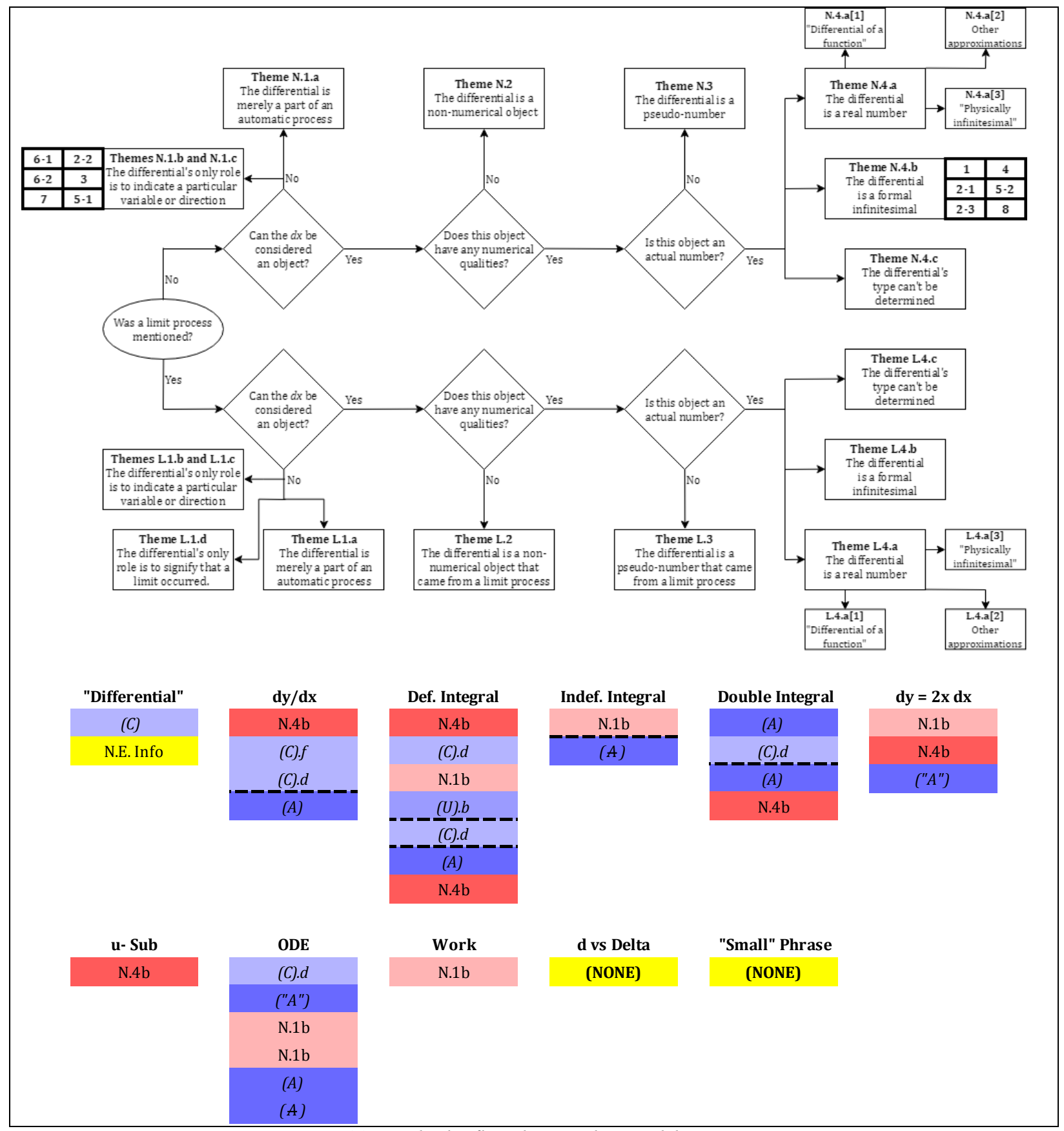

Figure 20: Zaphod's flowchart and spreadsheet 
Okay, so in part of my masters I studied nonstandard analysis, so if I'm thinking about differentials as objects with size, I think about them as infinitesimal elements. So I think about them as literal infinitesimals in the Abraham Robinson sense.

Second, while Gustav only used the word "limit" once during his interview, Zaphod used it ten times, but usually to describe how limits are not how he views differentials. When discussing Leibniz derivative notation, he initially said "Well, my immediate reaction is that it's $\lim _{h \rightarrow 0} \frac{f(x+h)-f(x)}{h}$. So if someone's asking me this question, I assume that it's a definitional question and that's what I would say,” and later said

I usually think of them symbolically, but I would be thinking about the definition of a derivative as a limit without the limit part. So I'd be looking at the difference in the output of the function over small time changes on the top. And on the bottom would be the difference in the inputs.

To me, this implies that he only mentions limits when he perceives that he needs to give a commonly-used definition; when he is left to his own devices, then differentials are formal infinitesimals. This implication can also be seen by the amount of "teaching" (C).d themes in his spreadsheet, as teaching would be a situation in which he might feel he needs to evoke a different part of his concept image.

However, not all of Zaphod's differentials were formal infinitesimals. For the solvable ODE, "Work" problem, and indefinite integral, Zaphod was comfortable viewing differentials as variable indicators (Theme N.1b). In the separable ODE, Zaphod was the only interview subject to mention the idea of a differential operator, describing the left-hand side of the separable ODE as the operator $\frac{d}{d t}$ applied to $\tau$, instead of the ratio $\frac{d \tau}{d t}$. Despite the idea of "differential operator" not occurring during my first round of interviews, I believe that it still fits within my framework 
as an example of "variable indicator". While this interpretation loses the differential " $d \tau$," Zaphod still stated that the role of the " $d t$ " in the operator $\frac{d}{d t}$ was to indicate the variable of differentiation.

\section{Summaries of Each Expression}

This section will briefly discuss both the main findings from each expression used in the interview protocol, and comparisons of the expressions that contained similar notations. I will address these themes in a different order than the order used in the interview protocol in order to group expressions that contain similar notations together: Leibniz derivative notation $\frac{d y}{d x}$ with the separable ODE that contains $\frac{d \tau}{d t}$, the generic definite integral presented without context with the "Work" problem that contains a definite integral, and the expression $d y=2 x d x$ with the "integration by substitution" problem, which contains the substitution $d u=\frac{1}{2 \sqrt{t}} d t$.

\section{The Initial "Differential" Question}

My intent in asking the initial question "What does the word 'differential' mean to you?" was to receive answers that could be used as a baseline with which to compare the later answers given to questions about specific differentials. In practice, however, such comparisons were rare, because most interview subjects gave basic, non-detailed answers to this initial question. Seven of the ten interview subjects gave responses that contained some simple variation on "it is small," which, by itself, is not enough to assign it a particular theme. , as the word "small" could be used to describe many themes on my list. Not only are there small real numbers and hyperreal infinitesimal numbers (Themes N.4a and N.4b, respectively), but "small" could also be a numerical quality found in a pseudo-number (Theme N.3 or L.3).

I would surmise that most of these initial, poorly-defined "small" ideas were merely placeholders that were later unpacked into more-complex ideas. While individuals might have rich 


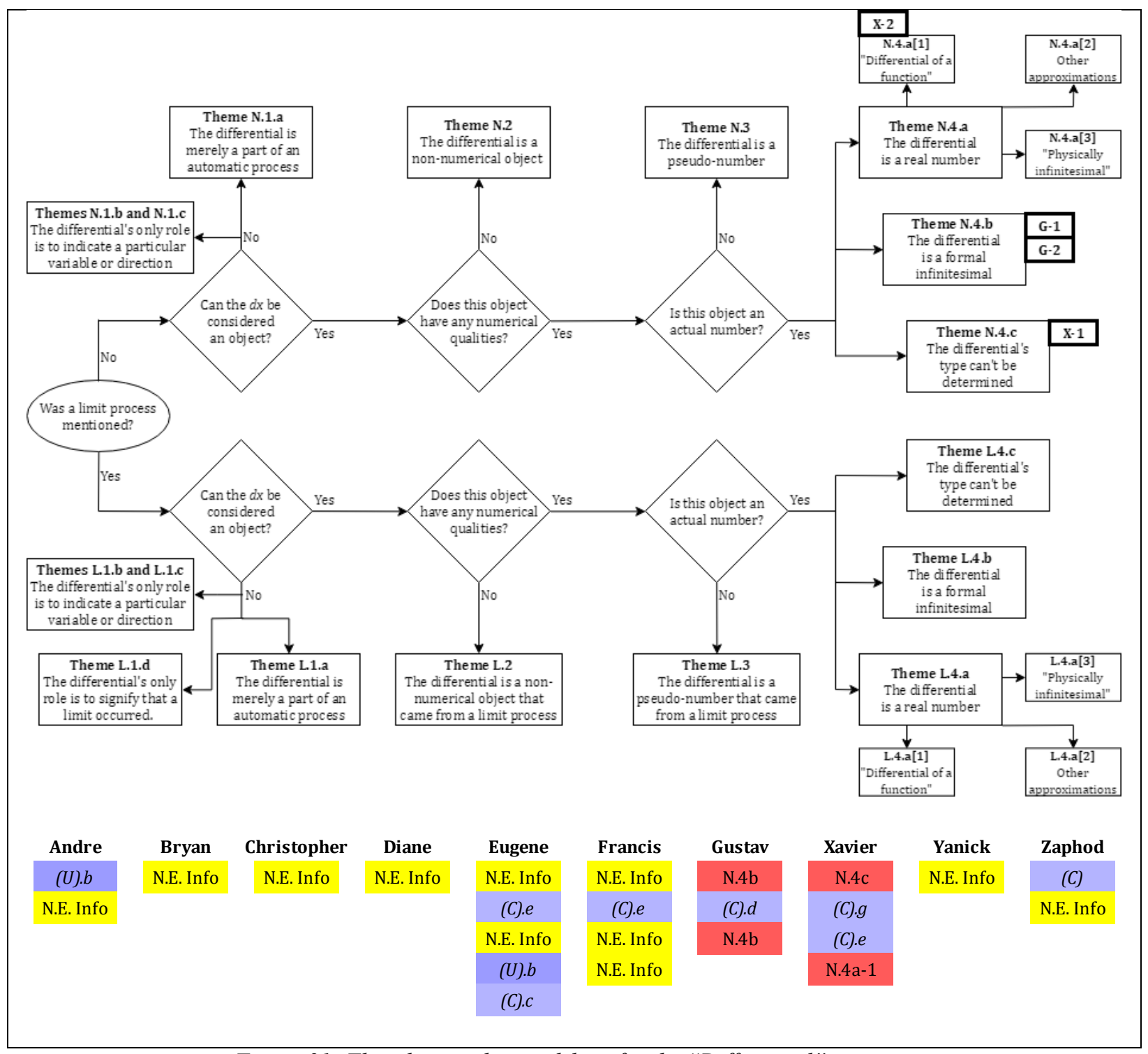

Figure 21: Flowchart and spreadsheet for the "Differential" question

and complex concept images for differentials, I believe that these images are rarely explored to their fullest in the everyday lives of most mathematicians. Thus, most of the interview subjects' initial responses consisted of merely a quick, surface-level idea of "small." As the interview progressed, the interview subjects got opportunities to not only think more deeply about differentials than usual, but also to attempt to vocalize and articulate their thoughts, after which more complex personal concept images emerged. These initial incomplete explanations are characterized by yellow "Not Enough Information" cells in the spreadsheets. 
Gustav and Xavier were the only interview subjects to provide enough additional detail so that their answers could be assigned to a specific theme. Gustav's initial remark was that differentials were "infinitely small," but he also mentioned Leibniz and Robinson, suggesting that his idea of "infinitely small” aligns with nonstandard analysis' formal infinitesimal values. Xavier gave his definition of a differential as the value $f^{\prime}\left(x_{0}\right) d x$, and while he did not explicitly say that the $d x$ in this definition was a real or hyperreal infinitesimal number, we can infer that it is at least some well-defined number, excusing it from the "pseudo-number" idea and placing it within the "well-defined value" part of my theme list. Finally, Zaphod's response to this question did not include any idea of "small." Rather, he stated that the word "differential" causes him to think only of the specific symbol $d x$ or $d y$, with the context of the particular expression determining what he would think next.

\section{The Interview Subjects' "Small” Phrase}

The questions about the interview subjects" particular phrase used to describe "smallness" were asked at different points in each interview, depending on when "smallness" was first mentioned. This mostly occurred during discussion of Leibniz derivative notation (for Christopher, Diane, Francis, Gustav, and Yanick), but it also occurred before Leibniz derivative notation (André), during discussion of definite integrals (Brian and Xavier) and the discussion of double integrals (Eugene). The distribution of themes in the flowchart and collection of colors in the spreadsheet suggest that one precise meaning of a phrase like "infinitesimally small" would be impossible to find, as my interview subjects' views varied widely.

Six of the interview subjects (Christopher, Diane, Eugene, Francis, Xavier, and Yanick) were not able to formally quantify the phrases they used. It is interesting to me that five of these six were the only ones to describe their phrases in terms of limits. Christopher and Xavier described 


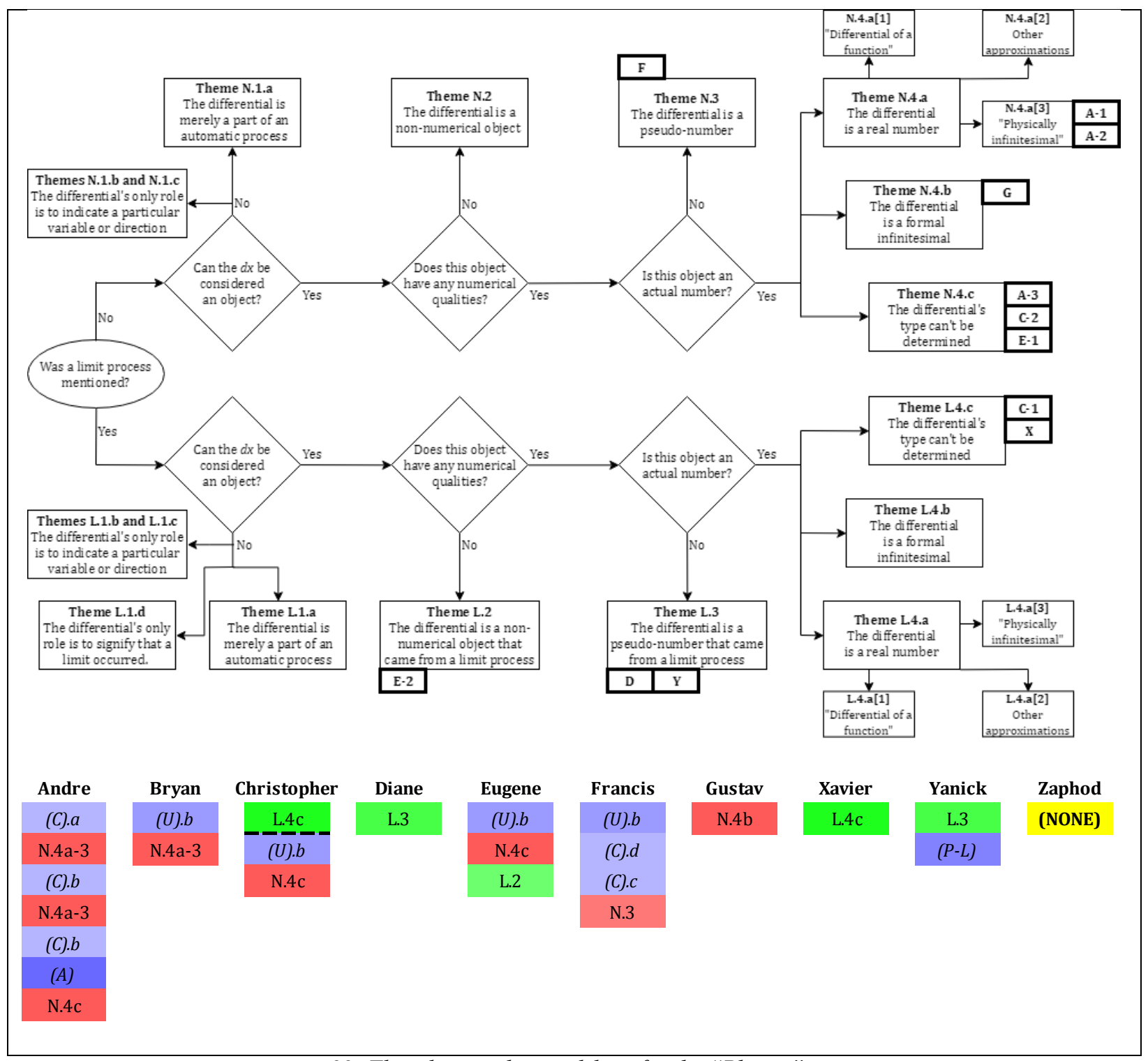

Figure 22: Flowchart and spreadsheet for the "Phrase" question

their phrases as the process of a limit, with Christopher using the active tense "limiting" to describe his "very, very small" as "a limiting relationship among very, very small quantities," and Xavier describing "infinitesimal” as "a process in which you take a quantity to the limit zero." In contrast, Diane, Eugene, and Yanick described their phrases as the result of a limit process. Diane specifically saying that "infinitely small" was "the result of a limiting process," Eugene describing 
“infinitesimal" as "somehow after [a limiting process is] all done and packaged," and Yanick describing "infinitely small" as "physical changes getting smaller and smaller" with $d x$ being "the smallest change," implying a finished limit. Francis did not mention limits when discussing "infinitesimally small," stating that it "is really just a concept ... can we quantify it? No."

The remaining interview subjects, however, were able to define their phrases more exactly. André and Bryan described their phrases as representing real numbers in manners akin to Courant and John (1965). André defined "infinitesimally small" specifically as "smaller than ... any relevant quantity in the problem," while Bryan used phrases like "small enough" and "relatively small," saying that these phrases "are always real numbers." Gustav and Zaphod mentioned hyperreal infinitesimal numbers as found in nonstandard analysis. Gustav said the aforementioned quote "Well, I can give you the definition: that's smaller than any $\frac{1}{n}$ and greater than zero. That's kind of positive infinitesimal" during his discussion of the term "infinitesimal," and while Zaphod did not ever specifically discuss any particular "small" phrase during his interview, he repeatedly stated during his interview that his personal use of the word "infinitesimal" was meant to convey Robinson's nonstandard hyperreal infinitesimals.

I find it interesting that Bryan and Christopher each stated that they, respectively, "try not to" or "usually don't" use such phrases because they can't be, respectively, "made precise" or are “too vague.” While Bryan does subscribe to Courant and John's (1965) "physically infinitesimal," he notes that the idea of "small" can vary from problem to problem: "If we're talking about astronomical stuff, you know, the change in $x$ being small could mean 100,000 miles, but when you're talking about molecules, you know, the change in $x$ can not be 100,000 miles, it's one Ångström.” 


\section{Differences between $d x$ and $\Delta x$}

Like the "small" phrase, the difference between $d x$ and $\Delta x$ was addressed at different times throughout the interviews, as shown in Table 6 below. To explain the last two rows: Eugene and Zaphod did not mention $\Delta x$ at any time during their interview, and while I had time to ask Zaphod about $\Delta x$ at the end of his interview, time constraints prohibited me from asking Eugene.

Overall, there seems to be no correlation between in which expression the idea of $\Delta x$ was first mentioned and how the $d x$ in those expressions was interpreted. Most of the responses (André's, Christopher's, Diane's, Francis', Yanick’s, and Zaphod's) were variations of the idea that an initial $\Delta x$ represented a finite quantity that could be measured, while the creation of a $d x$ involved some sort of limit applied to the $\Delta x$. Some explanations of the limits applied to the $\Delta x$ seemed confusing and even erroneous to me. For example, André described the $d x$ as "what's left of $\Delta x$ after it goes to zero" and Diane said that the $d x$ is "kind of the end result" of $\Delta x$ going to zero. These explanations seem to imply that, even though a limit process has gone to zero, there is still something remaining, which seems to contradict that $\Delta x$ has really proceeded to zero. Similarly, Diane, Francis, and Yanick each mentioned the idea that the $d x$ was the result of $\lim _{\Delta x \rightarrow 0} \Delta x$. This also seems contradictory, as by the laws of limits, this result should be equal to zero

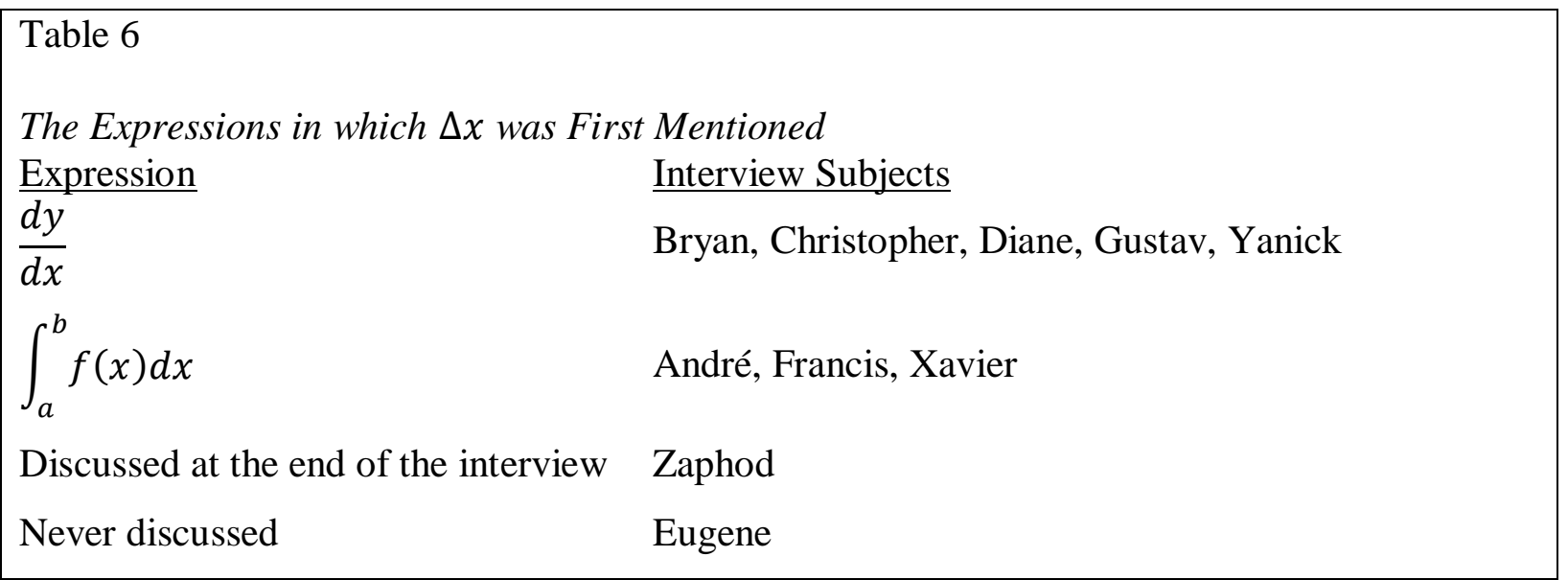


and not have a nonzero result (although, to match what she said above, Diane did qualify her version of this with a "sort of.") Other descriptions were less confusing, if not detailed. Christopher said that "there's some sort of limit involved" when one writes a $d x$, which is what differentiates a $\Delta x$ and a $d x$. Francis and Yanick both described the $d x$ as a theoretical concept, which differentiates it from a quantifiable $\Delta x$.

The other responses did not mention limits. Gustav, as he did before, described $d x$ in the style of nonstandard analysis: greater than zero yet smaller than any $\frac{1}{n}, n \in \mathbb{N}$, and thus different from the real-valued $\Delta x$. Bryan and Xavier at times held notions that there were no differences

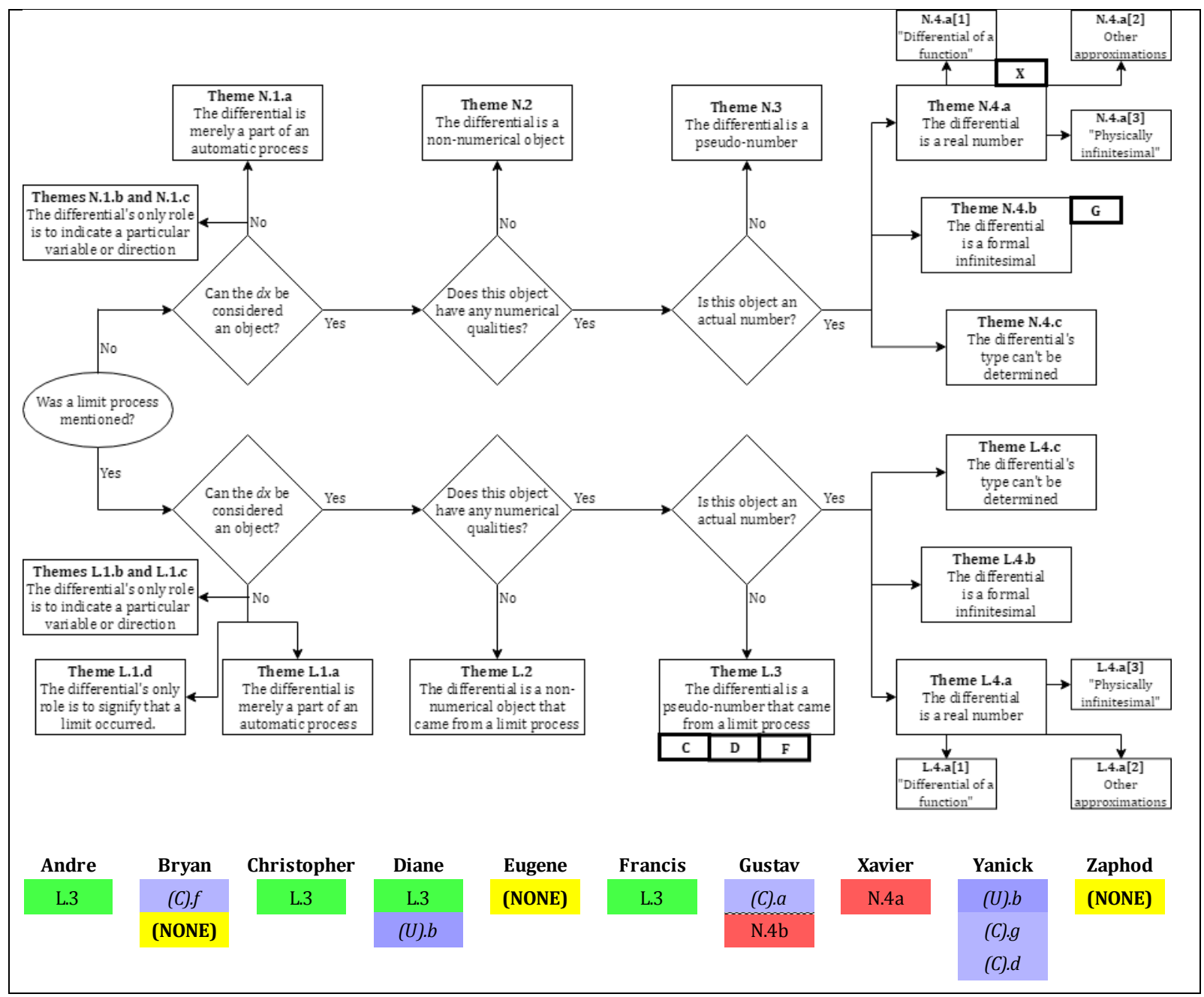

Figure 23: Flowchart and spreadsheet for the "Delta" question 
between $d x$ and $\Delta x$. Xavier initially described the $d x$ in a definite integral as the difference $x_{j}-$ $x_{j-1}$, a definition I would have expected for a $\Delta x$. Bryan directly said at one point that there was no difference between a $d x$ and a $\Delta x$, and illustrated this idea that these two notations were interchangeable when describing a definite integral as " $f$ multiplied by a little $\Delta x$ or $d x$." The fact that Bryan had no specific definition dedicated to a $d x$, instead viewing the terms " $d x$ " and " $\Delta x$ " as interchangeable, is what prompted the yellow "None" in this spreadsheet.

\section{Leibniz Derivative Notation $\frac{d y}{d x}$}

This expression was the first expression discussed during each interview, and there were multiple interpretations given for it, which can be seen by the summary given in Table 7 and the amount and variation of colors found in Figure 24. Each interpretation is more fully-explored below.

The first interpretation that I mention is the interpretation that this symbol is not constructed as a ratio of two separate entities $d y$ and $d x$, but is instead thought of as one symbol. Hans Freudenthal once stated that there was no meaning to these particular $d y$ and $d x$ :

\begin{tabular}{|ll}
\hline Table 7 & \\
Summary of Interview Subjects' Views of the Differentials in $\frac{d y}{d x}$ & $\frac{\text { Interview Subject(s) }}{\text { View }}$ \\
Only a Variable Indicator & Bryan, Eugene, Francis \\
Real, but "Physically Infinitesimal," Numbers & André \\
Formal, Hyperreal Infinitesimal Numbers & Gustav, Zaphod \\
Some Measurable Value, but the Specific Type is Unclear & Christopher, Xavier \\
Pseudo-Numbers Arising from a Limit Process & Diane \\
Pseudo-Numbers Not Arising from a Limit Process & Yanick
\end{tabular}


If $d y$ and $d x$ occur only in the combination $\frac{d y}{d x}$, or under the integral sign after the integrand, the question as to what $d x$ and $d y$ mean individually is as meaningful as to ask what the "l', "'o", "'g”' in "'log'” mean (Freudenthal, 1973, p. 550).

It was my initial belief that any interview subjects who viewed this expression as one symbol would also find the individual $d y$ and $d x$ meaningless. However, all of my interview subjects who held the "one-symbol" view, Bryan, Eugene, and Francis, stated some variation of the idea that this expression showed that $y$ was the dependent variable that relied on the independent variable $x$. An example of this is Eugene's statement that "this symbol usually occurs in the context where $y$ is a function of $x$ " Thus, I would suggest that, even if one does not view this expression as a ratio but still discusses the dependence of the variables, then the individual $d y$ and $d x$ are at least serving as variable indicators (Theme N.1b).

The most common view of the individual $d y$ and $d x$ however, was that of some measurable value, as shown by the number of themes in the upper-right of the flowchart and number of dark red themes in the spreadsheet. Some of these measurable values were explicitly defined. André described the value of these differentials as akin to Courant and John's (1965) "physically infinitesimal" values and thus real numbers:

They're very small compared to - not so much to $x$, but more like to the scales at which $x$ or $y$ varies significantly. So this could be, you know, this here [points to a spot on the $x$-axis in one of his drawings] could be $52,000,000$, and this [points at a second $x$-value] could be $52,000,000.1$

Gustav and Zaphod described the value of these differentials as formal, nonstandard, hyperreal infinitesimals, with Gustav stating "I understand, knowing what I know from the history, 


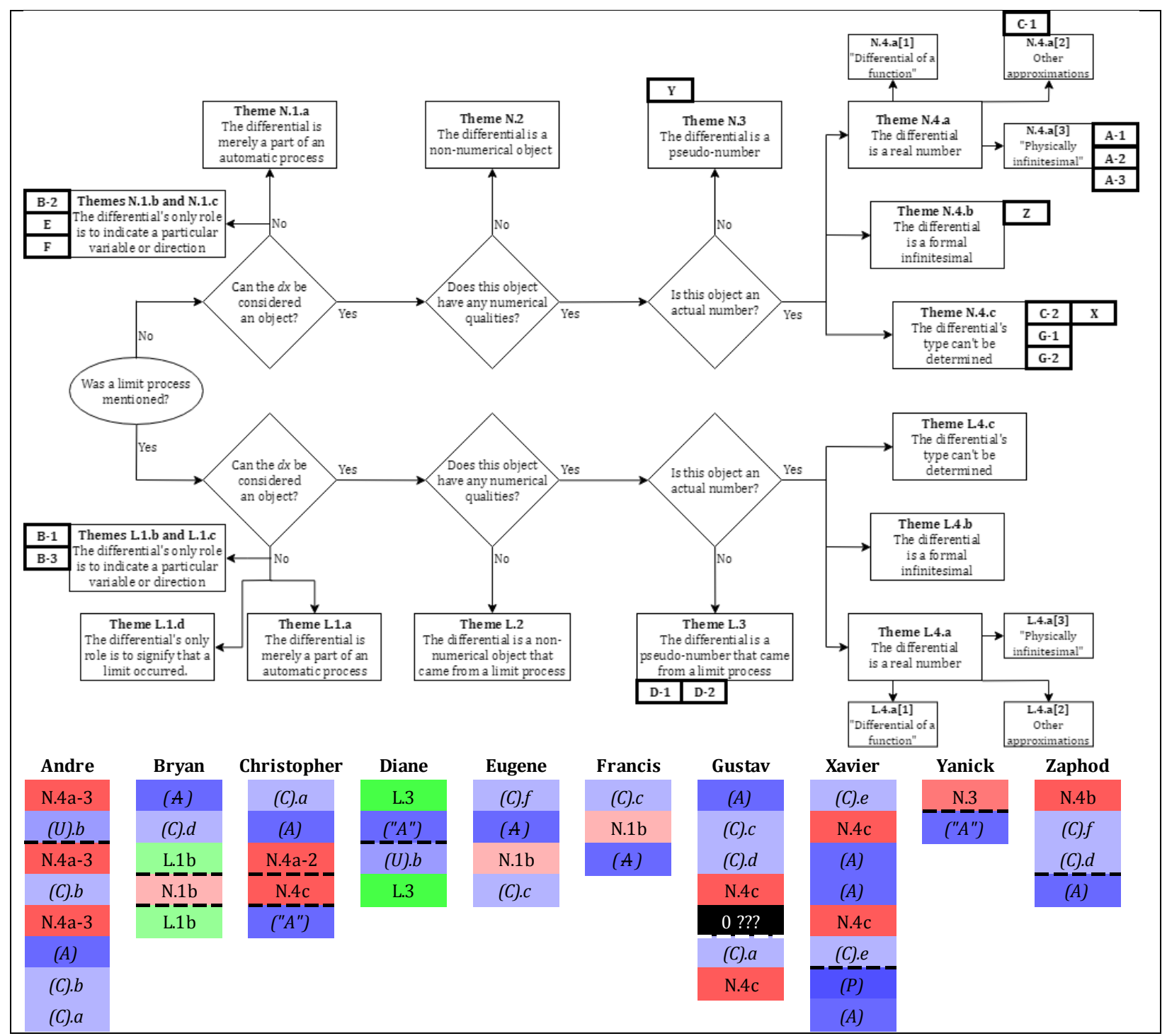

Figure 24: Flowchart and spreadsheet for $\frac{d y}{d x}$

that $\left[\frac{d y}{d x}\right]$ can be considered a ratio of infinitesimals," and Zaphod stating "if I'm thinking about differentials as objects with size, I think about them as infinitesimal elements. So I think about them as literal infinitesimals in the Abraham Robinsons sense."

Christopher and Xavier described these values in ways that imply that they are well-defined and measurable, but, unlike the three aforementioned interview subjects, they did not provide enough context clues to ascertain whether these values were real or hyperreal. Specifically, Christopher described the $d y$ and $d x$ as being legs of a right triangle, with the hypotenuse being a 
small portion of the function's curve magnified enough so that it appears linear. However, while the value right triangle legs can be assumed to be real numbers, Keisler (2012) uses similarlyconstructed right triangles but with infinitesimal-valued legs. Thus, without further explanation that I did not obtain during his interview, Christopher's differentials must remain measurable but not well-defined. Similarly, Xavier's description of $d y$ as being equal to $f^{\prime}\left(x_{0}\right) d x$ is also not specific enough, as the multiplication in Xavier's definition can also be done with hyperreal infinitesimals as well as real numbers. The other interpretation was of these differentials as pseudonumbers. Diane and Yanick both used the phrase "infinitely small" and while both admitted that this phrase was imprecise, Diane invoked the idea of this "infinitely small" coming from a limit process applied to a measurable $\Delta y$ and $\Delta x$, where Yanick did not.

The only other striking feature of the themes found in this expression was the larger-thannormal percentage of Tier 2 themes relative to all of the other expressions, as noted by the number of blue cells in Figure 24. There were discussions about viewing differential expressions in a variety of contexts: historical views of calculus, physical and physics-based contexts, how nonstandard analysis can impact one's views, the various meanings of the word "differential," and how expressions containing two differentials might be viewed differently than expressions containing one differential. Subjects also discussed the efficacy of thinking (or not thinking) of this symbol as a ratio, and the uncertainty that they might have about the interpretations of differential-based expressions. While it is possible that Leibniz derivative notation is an expression that naturally lends itself to these kinds of thoughts, a simpler reason for the high percentage of Tier 2 themes might be the position of this expression at the beginning of the interviews. Perhaps toward the beginning of interviews, the interview subjects might have been more inclined to discuss their views in greater detail because of their initial interest and excitement. As the interview 
continued, perhaps interest and excitement waned, and less-complete (and thus less-context-based) opinions resulted.

\section{The Separable ODE $\frac{d \tau}{d t}=-k \tau$}

The first expression $\frac{d y}{d x}$ was presented to the interview subjects without any explanation of what might be represented by the $x$ and $y$. In contrast, this ODE contained the notation $\frac{d \tau}{d t}$, in which $\tau$ and $t$ represented the physical quantities of temperature and time, respectively. Since temperature and time are measured with well-defined values, I initially assumed that the differentials in $\frac{d \tau}{d t}$ would be categorized by more "tangible" themes than the differentials in $\frac{d y}{d x}$. Specifically, compared to the flowchart and spreadsheet for Leibniz derivative notation, I expected more themes on the right side of this expression's flowchart and more darker red themes in this expression's spreadsheet. However, this assumed shift toward "more tangibility" did not happen for most of the interview subjects, as many of them viewed the differentials in the initial $\frac{d y}{d x}$ and the later $\frac{d \tau}{d t}$ in similar ways. The assigning of similar themes to these two sets of differentials was not necessarily dependent on how one viewed the initial $d y$ and $d x$. It occurred for some of those who viewed the $d y$ and $d x$ as some measurable amounts (Christopher and Xavier), for those who viewed them as some sort of pseudo-numbers (Diane and Yanick), and for one of those who viewed them as variable indicators (Eugene).

The other five interview subjects either showed a little evidence for my "tangibility" hypothesis, or showed evidence that contradicted it. The hypothesis was somewhat met for Bryan and Francis, who at first viewed the $\frac{d y}{d x}$ as one ratio and not a quotient, but yet advocated the idea that one could "multiply by $d t$ " in order to solve the ODE. This action was not enthusiastically endorsed by either Bryan or Francis. Bryan said, "if I had to actually write a textbook or something, 


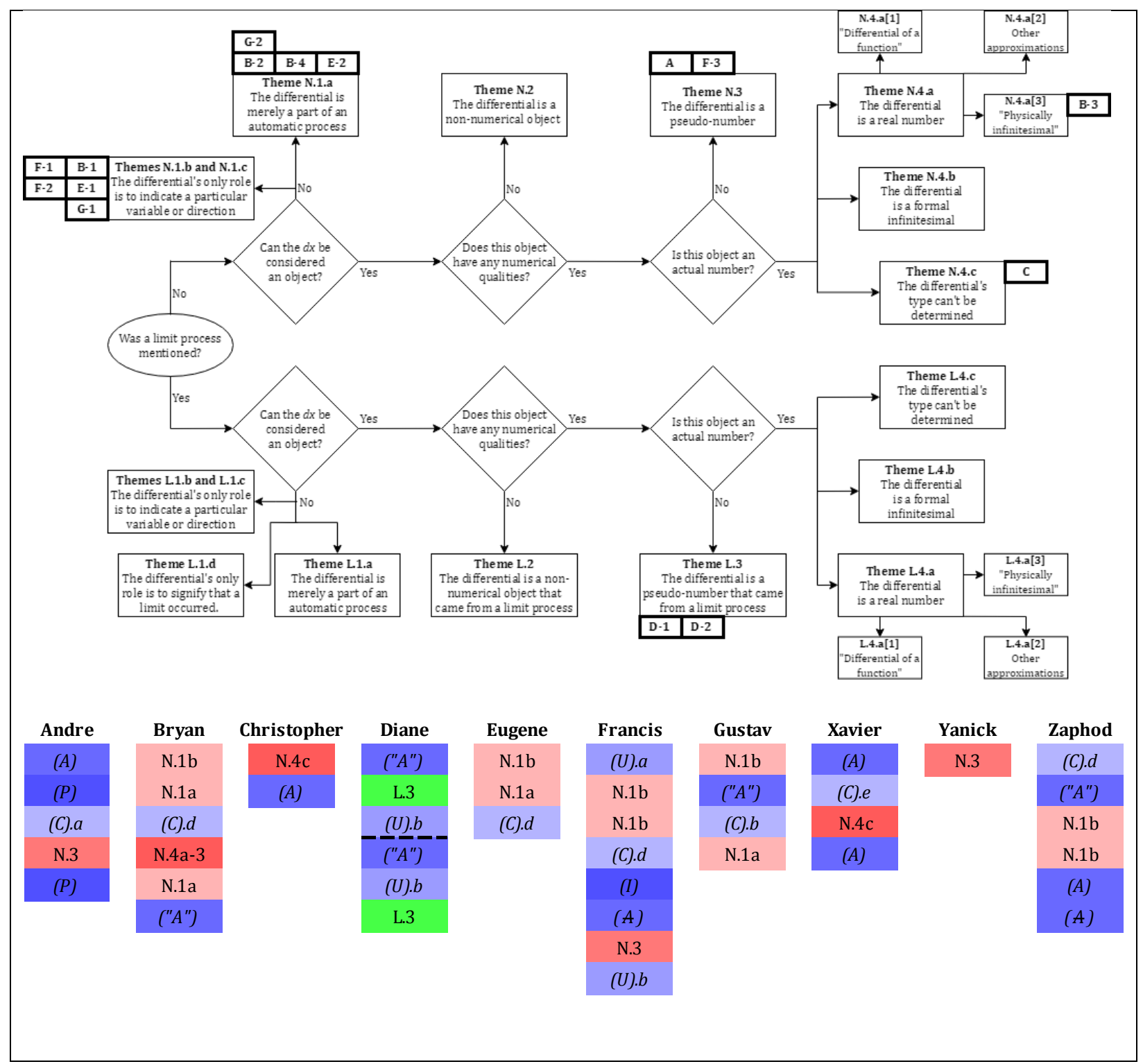

Figure 25: Flowchart and spreadsheet for the separable ODE

I'd have to think about it a little bit more about just saying 'multiply by $d t$ ' and then the $d t$ 's cancel", and Francis claimed, "I do have a discrepancy ... I'm not completely satisfied with this, that type of notation". This action assumes that $\frac{d \tau}{d t}$ at the very least acts like a quotient, thus endowing the individual $d \tau$ and $d t$ with a tangibility that their $d y$ and $d x$ do not have. On the other hand, Gustav's and Zaphod's remarks about the differentials in the ODE seemed to move in the opposite direction of my hypothesis. While their $d y$ and $d x$ were described as hyperreal 
infinitesimals, they spoke of the action of solving the ODE by "multiplying by $d t$ " as an automatic process, thus ignoring the $d \tau$ 's and $d t$ 's infinitesimal nature and turning them into only notational pieces of that automatic process. Similarly, André seemed to revert from describing the initial $d y$ and $d x$ as real values to describing the $d \tau$ and $d t$ as less concrete pseudo-numbers.

Aside from the differences in how the interview subjects viewed the differentials in the ODE, another set of differences was how they viewed the idea of "multiplying both sides by $d t$ " as a part of the solution method. The spreadsheet for the ODE (Figure 25) contains all three of the "Algebra" themes (A), ("A"), and (A), showing that there was a wide range of opinions on how to think about the idea of "multiplying by $d t$." Only Francis stated that this was not acceptable, saying that one should solve $\frac{d \tau}{d t}=-k \tau$ by integration $\left(\int \frac{1}{\tau} \frac{d \tau}{d t} d t=\int-k d t\right)$, as this removes the need for separating the initial $d t$ from the initial $d \tau$. Several interview subjects (Bryan, Diane, Eugene, and Gustav) mentioned that this separation was acceptable but heavily qualified this separation with phrases that spoke to their uncertainty that this was a valid mathematical operation, such as "be careful what it means" (Bryan) or "I would spend more time thinking about it" (Diane). André, Christopher, Xavier, and Yanick seemed to feel that such multiplication required no qualifiers. This is perhaps summarized by Christopher's description of differential equation modeling as "What happens over the next $d t$ ?", implying that, since this $d t$ is measurable, multiplying by it is perfectly valid.

On top of all of these conceptualizations for the differentials in the ODE, there were statements made by some interview subjects that were not differential-based at all. Upon being asked to solve the ODE, Bryan and Christopher stated that they would not teach nor expect their students to solve it by separation, preferring instead that they simply recognize it as an equation modeling exponential decay. This view might render any discussion of the differentials 
meaningless, outside of viewing them as variable indicators to denote the dependent and independent variables. Zaphod said that he interpreted the ODE as $\frac{d}{d t}(\tau)=-k t$, with " $\frac{d}{d t}$ " as a differentiation operator. Again, this view eliminates $d \tau$ as its own entity, and regulates $d t$ as a representative of the variable of differentiation.

\section{The Generic Definite Integral $\int_{a}^{b} f(x) d x$}

This expression was the second expression discussed in each interview and was presented immediately after Leibniz derivative notation. Because a good portion of the literature I cited in my literature review showed that there were many student conceptualizations of the differential found in a definite integral, it was not surprising to me that not only were there many expert conceptualizations of this differential, but several experts had multiple views of this differential as well. I summarize all such conceptualizations in Table 8.

Some of the differentials in the definite integral were described as limit-based, indicated by the green cells in the spreadsheet. André viewed this differential as an indicator that a limit process happened: “ $d x$ represents the fact that it was a Riemann sum." Diane viewed this differential as a reification of the limit process: "I think about it more just as, again, the result of a limiting process." To me, there is a nuanced distinction here: both interview subjects are stating that a limit was applied to a sum that contained $\Delta x$, but Diane's $d x$ is the transformation of the $\Delta x$ into a different, tangible object, whereas André's $d x$ is merely a symbol that states "this limit took place." There were more "numerical" differentials in definite integrals as well, which were already mentioned in the individual summaries: Christopher and Francis viewed them as limit-based pseudo-numbers: values that can't be quantified but can still be multiplied or used to define width, and one of the ways Xavier viewed them was as limit-based quantities that can be quantified. Similar variation happened within the non-limit-based themes. A common interpretation for the 


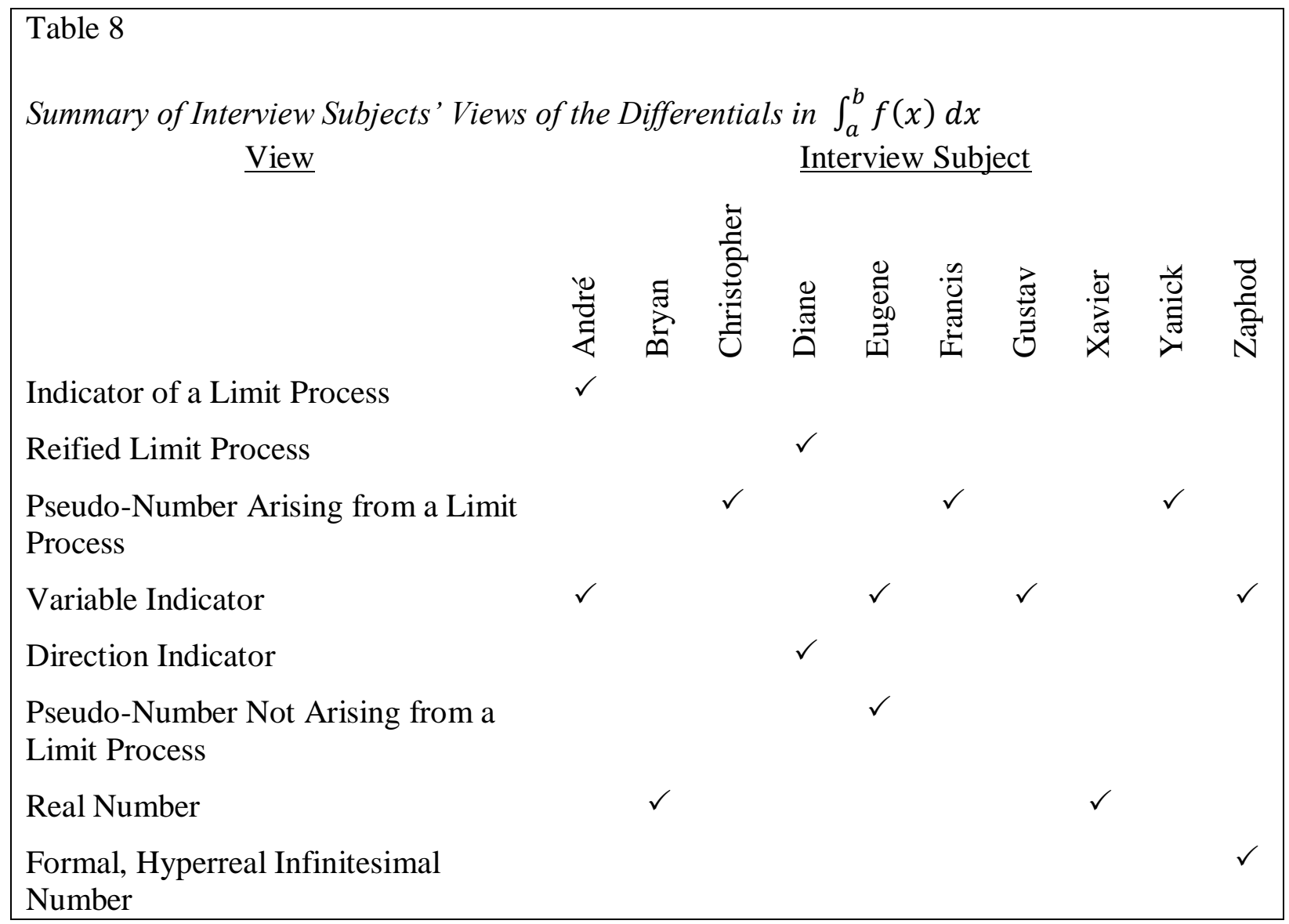

definite integral's differential was an indicator for the variable of integration. One of Eugene's views was of this differential as a pseudo-number, but a pseudo-number that did not come from a limit. Finally, Bryan and Zaphod viewed these as well-defined values: Bryan as "physically infinitesimal" real numbers and Zaphod as nonstandard analysis formal infinitesimals.

Some names in Table 8 had two checkmarks because the definite integral's differential was one in which some interview subjects' views changed depending upon the context. The definite integral's spreadsheet shows that Eugene and Zaphod expressed Theme (C).d, that differential views could change when teaching, while André expressed Theme (C).a, that differential views can change depending on one's view of the history of the notation. This explains how these three interview subjects could state multiple views for the definite integral's differential: 


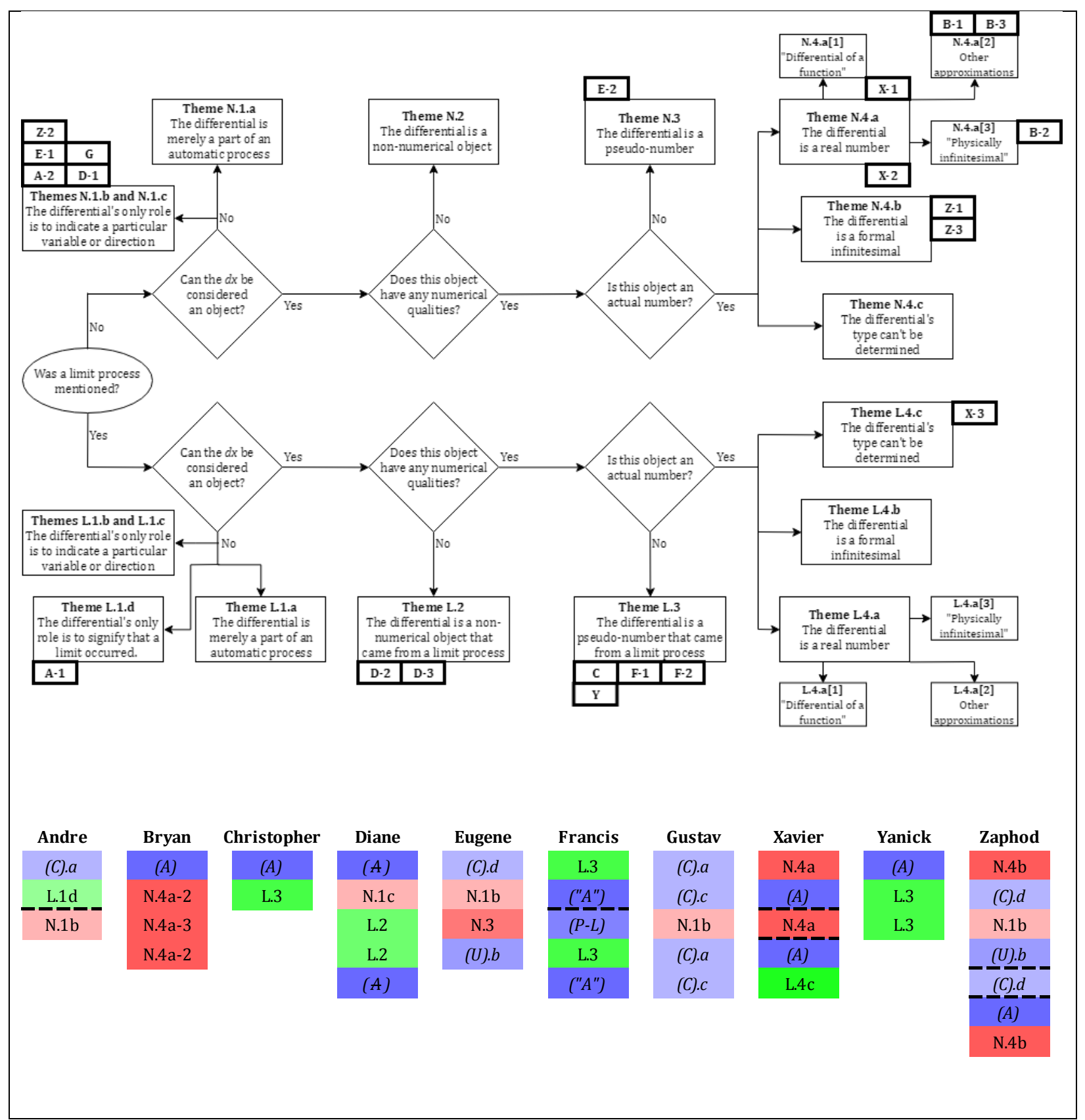

Figure 26: Flowchart and spreadsheet for $\int_{a}^{b} f(x) d x$

Eugene and Zaphod teach different views depending on the level of their students, while André can hold two different views depending on whether he is considering "historical" or "practical" viewpoints. 


\section{The "Work" Problem Containing the Integral $\int_{0}^{50} 700-3 x d x$}

Unlike the shift from Leibniz notation to the $\frac{d \tau}{d t}$ in the separable ODE, there was a general change in views from the generic definite integral to the specific definite integral in the "Work" problem. Overall, there was some movement away from limit-based themes and toward non-limitbased ones, and some movement away from more "ephemeral" differentials towards more "tangible" ones. These shifts can be seen in the greater concentration of themes to the right of this flowchart greater prevalence of darker shades of red in this spreadsheet as compared to the definite integral's flowchart and spreadsheet. However, these shifts were not absolute and did not occur in everyone's responses.

André and Christopher were interview subjects who moved from primarily limit-based responses for their generic definite integral to non-limit-based responses in the specific one. Specifically, André's descriptions went from the definite integral's differential as "the $d x$ represents that [the Riemann sum is] that sum when $\Delta x$ goes to zero" to the specific definite integral's differential as "I would have to consider the $d x$ represents the displacement." Christopher's definitions showed a similar progression, from the definite integral's differential's size as "really small and getting smaller ... as a limit" to the specific definite integral's differential described as "a small movement up the side of the building." Gustav similarly shifted his view of the generic definite integral's differential from the indicator of the variable of integration to, in his words, "I would remember this $[d x]$ as a displacement."

However, not everyone's views of the differentials in these two integrals shifted, and while some interview subjects' views shifted, that shift was not necessarily to a more "physical" differential. Both Diane and Francis viewed the differentials in each integral as limit-based objects and pseudo-numbers, respectively, while Eugene viewed both integral's differentials as variable 


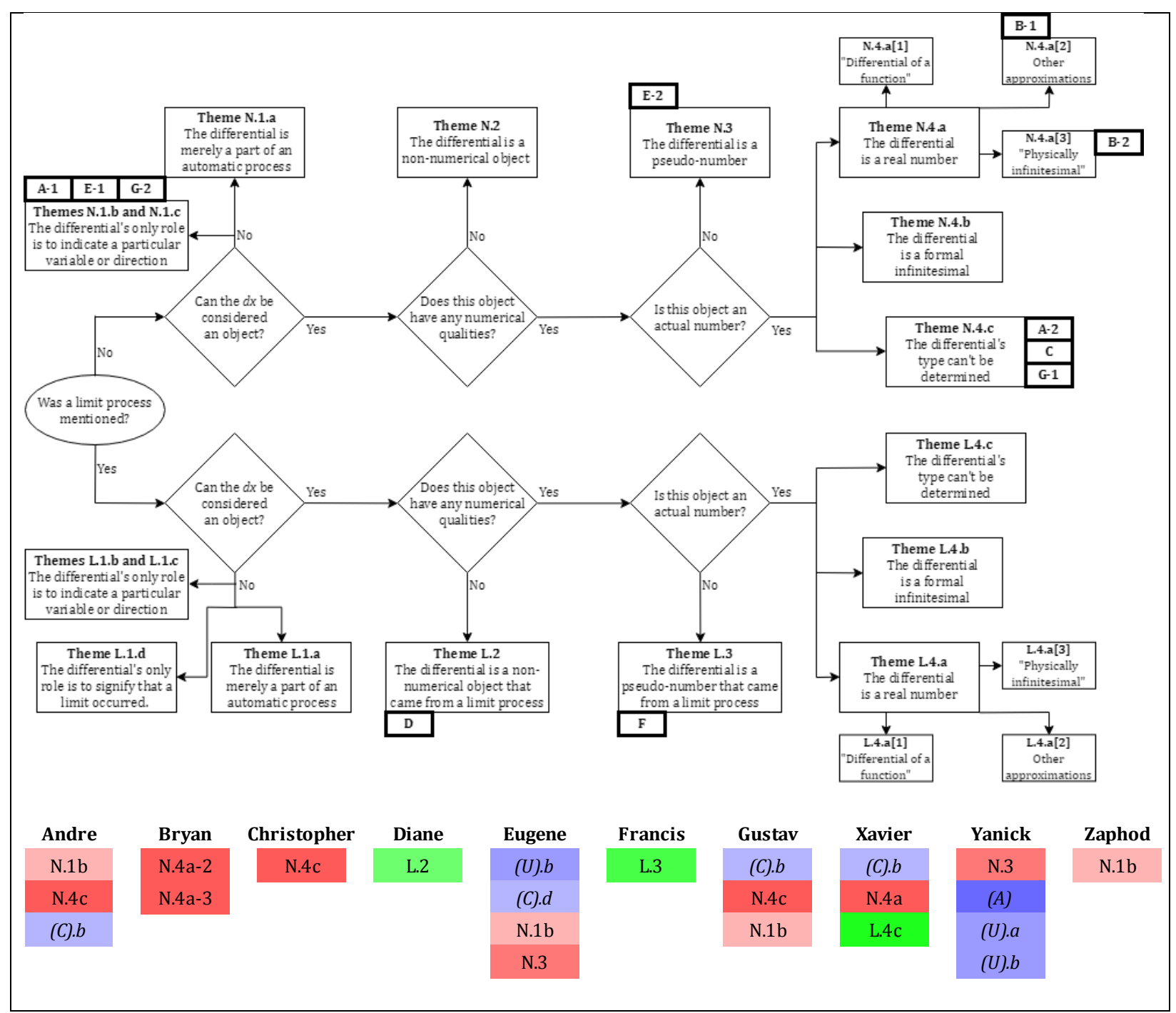

Figure 27: Flowchart and spreadsheet for the "Work" problem

indicators or pseudo-numbers, depending on the level of the students he would be teaching. The fact that the specific definite integral described a physical condition did not change their views. As an example of a different shift, Zaphod stated that the generic definite integral's differential was a formal hyperreal infinitesimal while the differential in the specific definite integral was primarily a variable indicator. However, this analysis may simply have resulted from incomplete questioning and exploration of his ideas, as he also mentioned the idea that the generic definite integral's differential could be thought of as a variable indicator. 
There was enough of this movement to suggest that, in this case, providing a physical context in which the integral can reside has an effect on the conceptualizations of the differential in that integral. In fact, André, Eugene, Gustav, and Xavier each mentioned some version of an idea that physicists and mathematicians might view this integral differently, utilizing by Theme (C).b. To give one example, André described this idea in terms of "realms": a "physics" realm in which the integral is created and the $d x$ has a physical quality, and a "mathematics" realm in which the integral is evaluated and the $d x$ merely indicates the variable of integration. This implies that any concept definition for the integral might contain different elements that are evoked or repressed depending on whether the integral is presented within a physical context.

\section{The Indefinite Integral $\int g(x) d x$}

This expression was presented at the same time as the definite integral, although most interview subjects discussed each integral separately. Of all of the differential-containing expressions in the interview protocol, the indefinite integral is the expression that contained the most uniform responses, which can be seen by the relatively simple flowchart and spreadsheet for this expression. Six out of the ten interview subjects (André, Diane, Eugene, Francis, Gustav, and Zaphod) stated that the purpose of the differential in a definite integral was to indicate the variable of integration, and nothing more. In general, interview subjects spent noticeably less time discussing this expression than discussing the definite and double integrals. This lesser amount of time and simpler theme distribution, however, might be attributed to the nature of this expression: Diane and Eugene both commented that, in a sense, the $d x$ in an indefinite integral is less important to a first-year calculus student since he or she has not yet experienced multiple integrals. As Diane said: 
It doesn't come into play so much in Calc. 1, because they generally have only one variable showing up, but when you have more variables or you have a variable that's a constant or something, then it's important to have that notation there. But in my mind, for the indefinite integral, it's literally just notation for the general antiderivative. $d x$ is mostly there to tell me what variable the change is - in the direction of

There were exceptions to this characterization of "variable indicator." Yanick thought for a while about how the indefinite integral's $d x$ might be conceptualized and

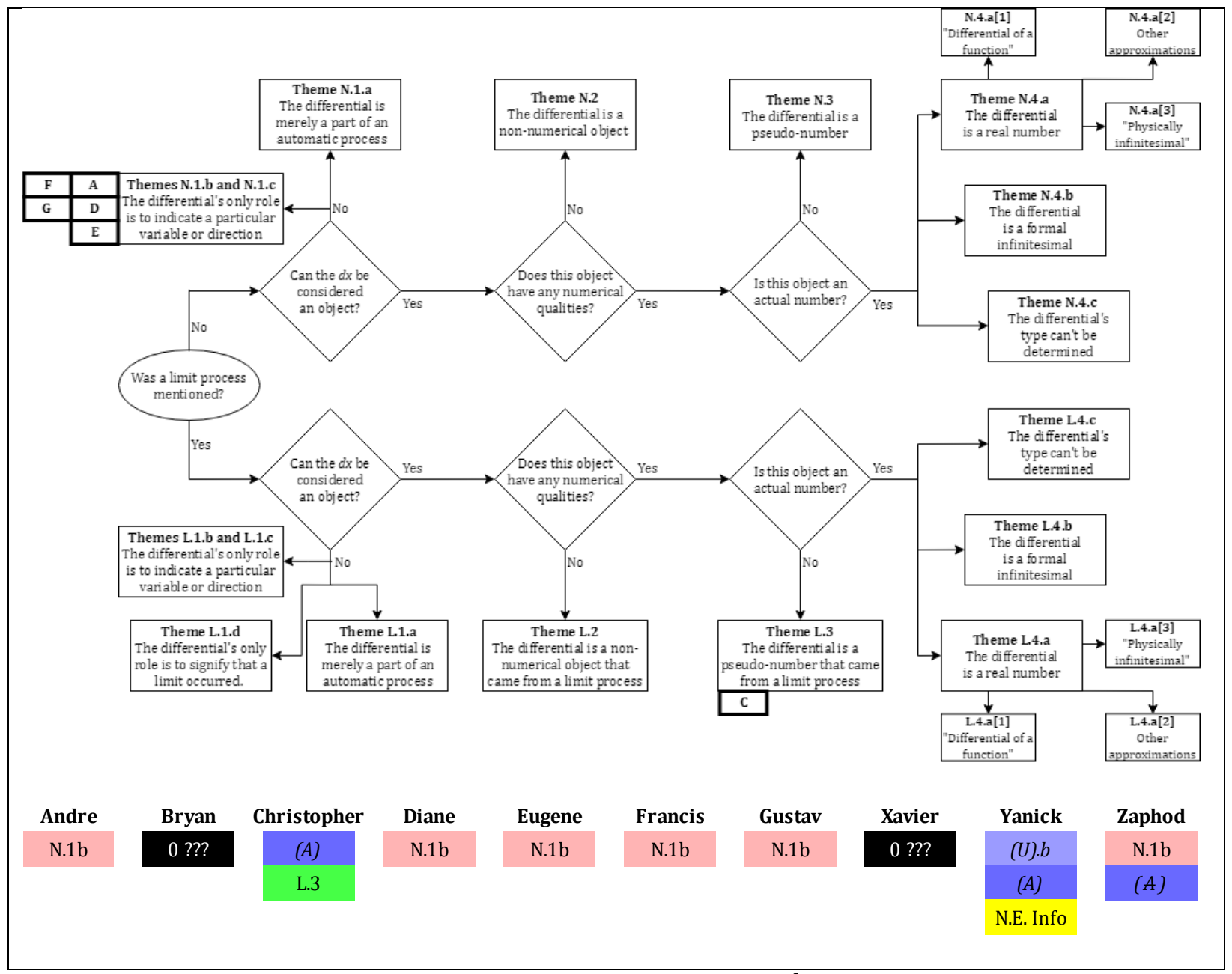

Figure 28: Flowchart and spreadsheet for $\int g(x) d x$ 
eventually stated that he needed to think more about it before committing to an answer. Bryan and Xavier made statements that suggested the entire expression (3) was a notation for "the antiderivative of $g$ " and thus, the $d x$, as a part of this notation, had no meaning by itself. Perhaps further questioning of these two might have elicited responses that contained a phrase like "in terms of $x$ " that could have shown a "variable indicator" theme, but on the other hand, Bryan stated "it could almost mean nothing," where further questioning confirmed that "it" referred to the indefinite integral's $d x$. Thus, I feel that "no meaning" is a viable option for this differential.

Finally, Christopher seemed to suggest an interpretation for the indefinite integral that matches Thompson's (1961) interpretation: the expression $d y=g(x) d x$ contains a $d x$, which measures a small horizontal displacement and is multiplied by $g(x)$ to form $d y$, a small vertical displacement. Thus, $y=\int d y=\int g(x) d x$ is the sum of all of these small vertical displacements, expressed as a function: "And you can sort of interpret it as a - it's an antiderivative in the sense that if you do take $g(x)$ times $d x$ and add it up, you'll get the increment in an antiderivative." However, it is fair to say that viewing this assessment as a definitive assessment may have happened because of imprecise questioning. Recognizing his description as similar to Thompson's, I immediately asked questions about possible graphical interpretations of the indefinite integral, as Thompson peppered his interpretation with graphical representations. As a result, I may not have given Christopher an adequate attempt to revisit the indefinite integral, in case he had any additional interpretations.

The Double Integral $\int_{0}^{1} \int_{2}^{3} f(x, y) d y d x$

This expression was presented immediately after the definite and indefinite integrals and the differentials in this expression are fairly easily described: the breakdown of themes for the differentials in the double integral are almost exactly the same as the breakdown of themes for the 
definite integral. I would hypothesize that the reason for this is because a double integral can be viewed as the combination of two definite integrals, and thus it is understandable that the collections of themes for the double integral and definite integral are near-identical. Indeed, during the discussions of double integrals, many interview subjects (André, Bryan. Francis, Xavier, Yanick, and Zaphod) stated variations of Yanick's idea that “And so you're doing the same [with the double integral] as you're doing with the definite integral up above.” This similarity can also

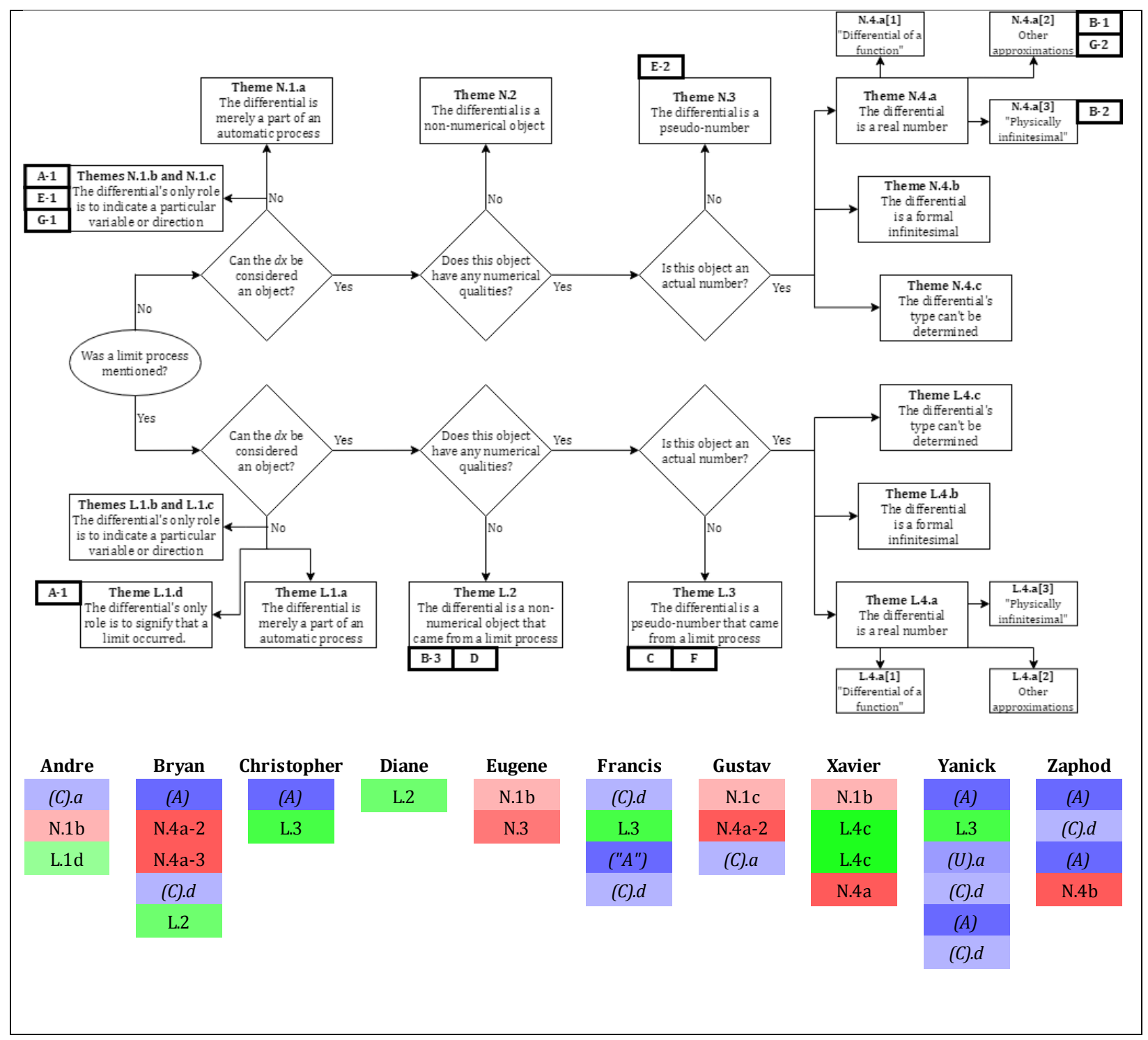

Figure 29: Flowchart and spreadsheet for $\int_{0}^{1} \int_{2}^{3} f(x, y) d y d x$ 
be seen by noting the resemblances between the double integral's and the definite integral's flowcharts and spreadsheets.

The only differences found between those two collections are minimal. Comparing the themes from the double integrals to the themes from the definite integrals, Xavier gained a "variable indicator" theme while Zaphod lost one. Gustav gained a theme of the double integral's differentials possibly being real numbers used for approximation, and Bryan gained a theme of the double integral's differentials as limit-based. However, each of these may not be actual differences between one's views of definite and double integrals but rather superficial differences that resulted from either incomplete questioning or whether the interview subject remembered to discuss every aspect of differentials for every expression. To use Bryan as an example: when discussing Leibniz derivative notation, he stated that he adopted a limit-based approach for that notation when he was teaching. When discussing the double integral, the topic of teaching came up again, and he made a similar statement that he uses limit-based themes for teaching. For whatever reason, he did not bring up teaching when discussing the definite integral. Perhaps it is possible that if he had done so, he might have also claimed a limit-based approach for those differentials that he did not claim during his interview.

\section{The Expression $d y=2 x d x$}

During some of my interviews, there were small amounts of uncertainty with this expression, usually a vocabulary issue regarding the word "differential." In my personal experience, expression (5) was called the "differential of a function" and the word "differential" was reserved for an individual $d y$ or $d x$. However, some interview subjects defined the word "differential" as expression (5). When asked the very first question of the interview "What does the word 'differential' mean to you?" these interview subjects responded by discussing this 
expression, which was noted in my spreadsheet by Theme (C).e. Since my definitions shaped how I designed my interview protocol, such discussion of expression (5) occurred much earlier than I had planned, and thus, some responses for this expression were pulled from the very beginnings of some interviews.

Two interview subjects had limit-based views of the differentials in this expression. Diane

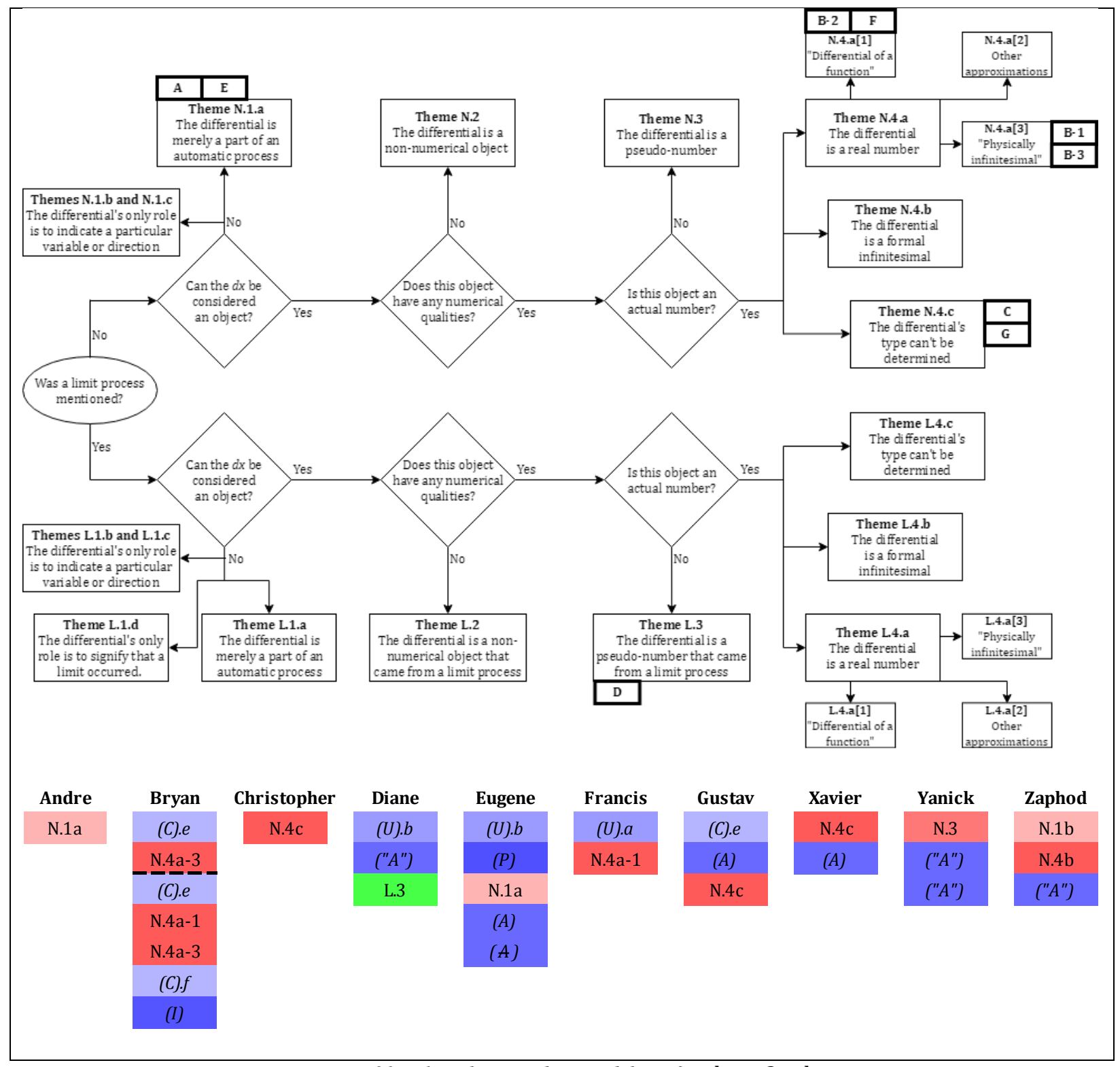

Figure 30: Flowchart and spreadsheet for $d y=2 x d x$ 
was the only interview subject to state that the differentials in this expression came from a limit process applied to Deltas, and Yanick viewed these differentials as limit-based pseudo-numbers. Among the other interview subjects who gave non-limit-based themes, however, there was a definite split in their views. On one end, perhaps owing to a "differential of a function" ideas, six interview subjects (Bryan, Christopher, Francis, Gustav, Xavier, and Zaphod) viewed these differentials as some type of well-defined number, usually in the "Linear Approximation" context described earlier: $\Delta y \approx d y=f^{\prime}(x) d x$, where $\Delta x=d x$, but $\Delta y \neq d y$. On the other end, André, Eugene, and Zaphod viewed these differentials as part of an automatic process that occurs without any justification. In other words, if given the expression $y=x^{2}$, one can just go ahead and write the expression $d y=2 x d x$ without thinking about why this can be done. Zaphod is listed in both groups since he began discussion of this statement with "This expression I could see appearing in a few different contexts," and included both remarks about automatic process and small amount. This split can be seen in the spreadsheet (Figure 30), via the mixture of darker and lighter shades of red.

\section{The Integration by Substitution}

I have already noted the shifts in the general tone of the differentials that happened both from Leibniz derivative notation $\frac{d y}{d x}$ to the ODE containing the expression $\frac{d \tau}{d t}$ and from the generic definite integral $\int_{a}^{b} f(x) d x$ to the "Work" problem's definite integral $\int_{0}^{50} 700-3 x d x$. In some interview subjects, I found shifts in the general tone of the differentials from the earlier, generic $d y=2 x d x$ to the specific $d u=\frac{1}{2 \sqrt{t}} d t$ used as a step in an integration by substitution. The interview subjects not listed below (André, Eugene, Xavier, Yanick, and Zaphod) had no such shifts, viewing the differentials in these two expressions similarly. 
This primary shift was in a direction away from "actual values" (Theme N.4) and toward the differentials having no meaning outside of being a part of a mindless process described as "If $u=\sqrt{t}$, then one can just automatically write $d u=\frac{1}{2 \sqrt{t}} d t$ " (represented by Theme N.1a). This shift was adopted by Bryan, Christopher, and Gustav. For example, Bryan stated that the $d x$ in $d y=2 x d x$ was "a relatively small number," but that, when using $d u=\frac{1}{2 \sqrt{t}} d t$ as a substitution

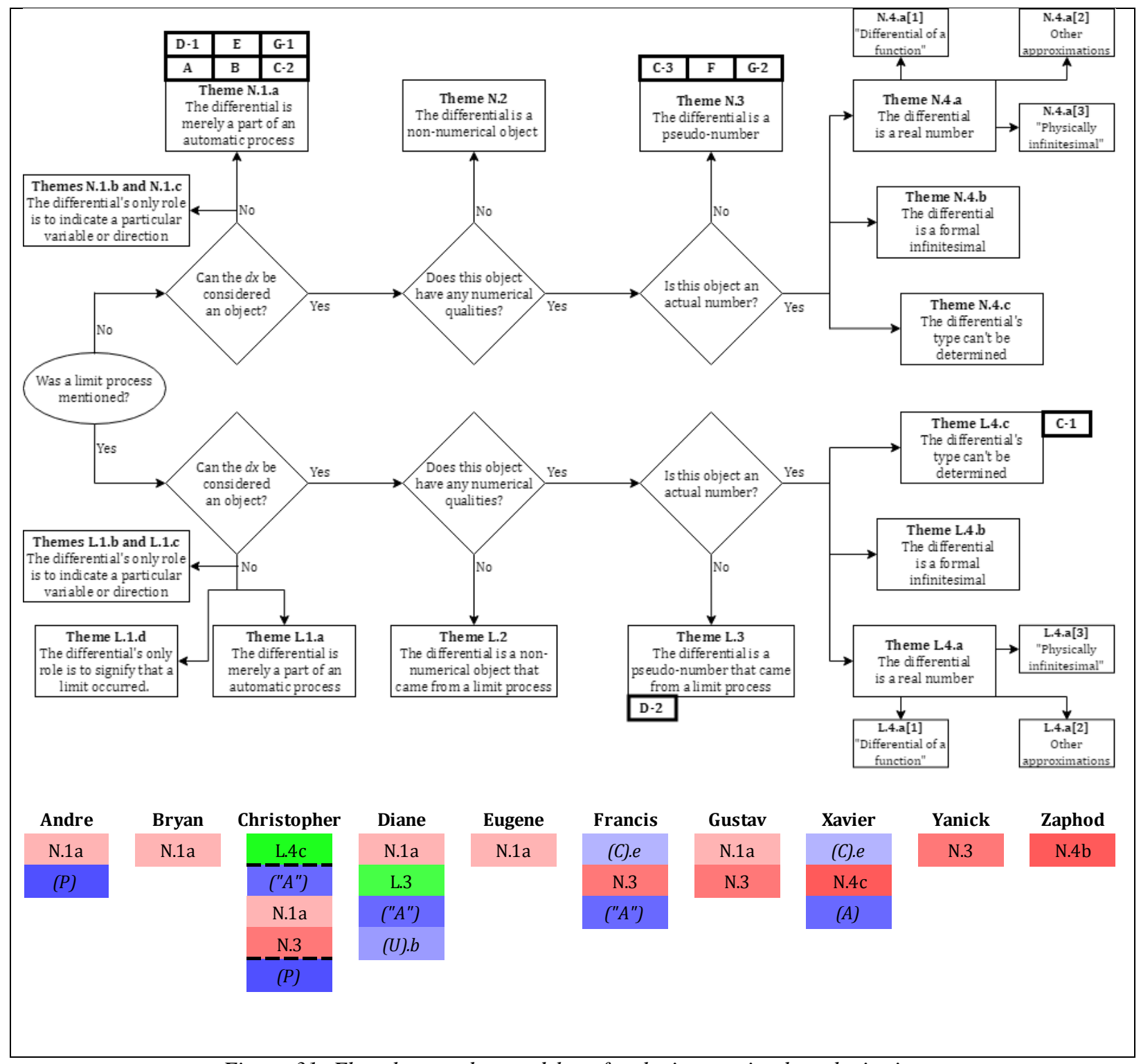

Figure 31: Flowchart and spreadsheet for the integration by substitution 
said, "To me, it's just mechanical," and "When I do it, I don't think about [the size of the differentials] at all." Diane had a similar shift, calling the substitution step "almost like a trick, or a notational thing that we can use to help us figure [the original integral] out," but her shift was not as pronounced as the others', since she never viewed the differentials in $d y=2 x d x$ as real numbers, but instead as limit-based pseudo-numbers.

A second shift by Christopher, Francis, and Gustav was from the earlier differentials as real numbers to the "substitution" differentials as pseudo-numbers. To use Francis as an example, it was stated in his section above that the differentials in $d y=2 x d x$ were the only differentials he viewed as real numbers. When describing the differentials in the substitution step, he noted that they had a relationship, confirming that they at least have some numerical qualities, but repeatedly called them "infinitesimal," which he later confirmed was a word that he used as a synonym for "unquantifiable." Both of these shifts can be seen by comparing this spreadsheet (Figure 31) and the previous one, and noting that the majority of themes in this spreadsheet are lighter-colored than the corresponding themes in the previous spreadsheet.

\section{Answering the Research Questions}

In this section, I will provide my answers to my three research questions. It is probably true that a quick glance at the summaries, flowcharts, and spreadsheets given in the previous section can give the reader the most basic answers to these questions, but I should like to expand on that material.

\section{How extensive are the concept images of differentials held by expert mathematicians?}

The two multiple-theme lists I have created from the results of my interviews show the vastness of the concept images held by my interview subjects in two different ways. The first is the breadth of the flowchart I created for the Tier 1 themes. As it currently exists, I have found 
eight main themes, and the total number of times each of the main themes was used by my interview subjects is shown in Figure 32. Even if one were to only look at the most common of these main themes and not any subthemes, one would still see that concept images can be very extensive, as the three most common themes describe differentials as the completely differing well-defined values, ephemeral ideas such as variable indicator or automatic process, and limitbased pseudo number.

Some of these eight themes are partitioned into enough subthemes so that any differential that is analyzed using my flowchart can end up in one out of a total of seventeen destinations. While some of these destinations were not reached by the descriptions given by my interview

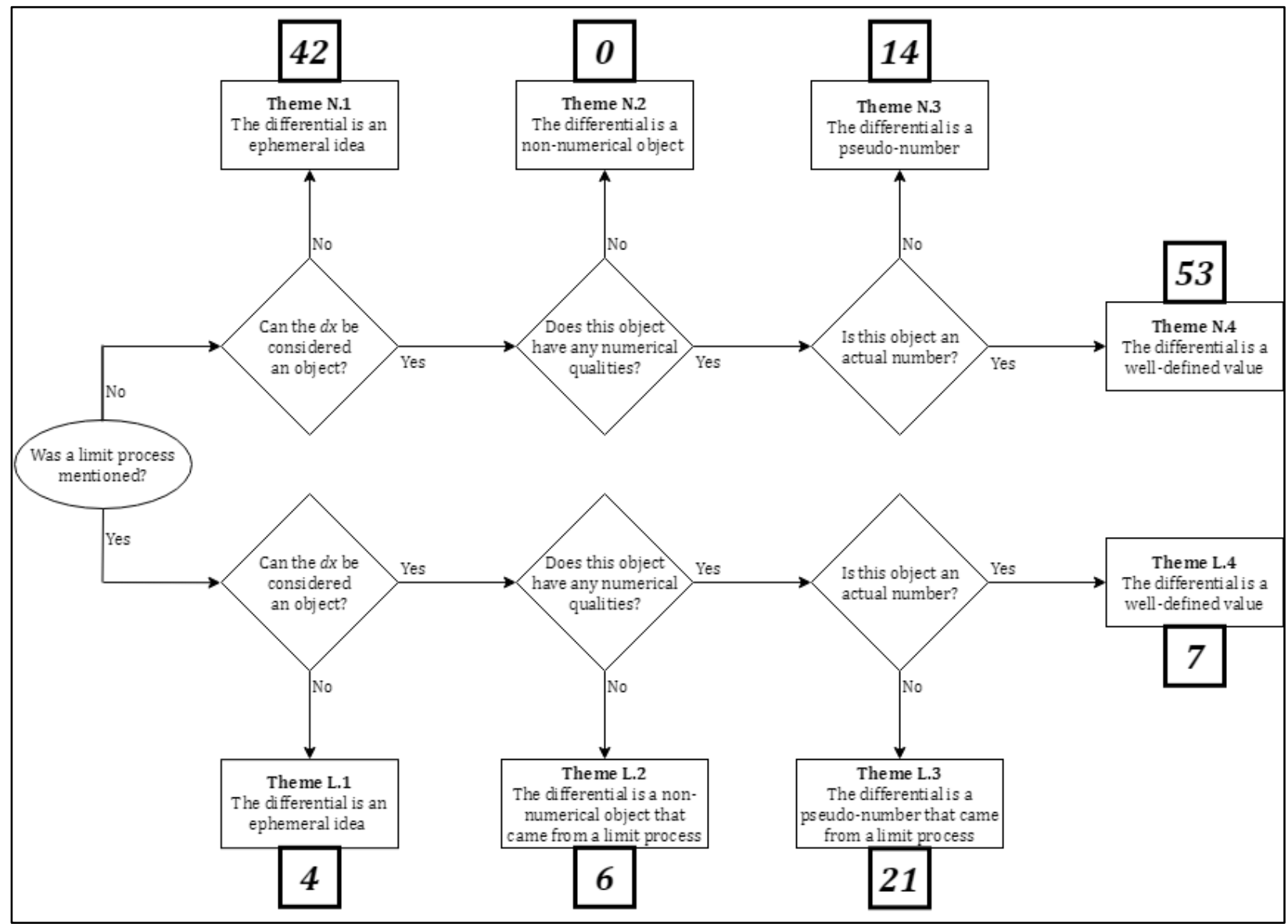

Figure 32: Total number of Tier 1 themes 
subjects (Examples being Themes N.2, L.1a, and L.4b), this does not mean that future interview subjects will never describe differentials that reach these destinations. It is also possible that future interview subjects might describe new subthemes that were not found in my dataset. The existence of at least seventeen possible destinations reinforces how extensive concept images of differentials can be.

The second is the prevalence of what I have been calling my "Tier 2" themes, and the total number of times each Tier 2 theme was used is shown in Figure 33. Before beginning this dissertation, I assumed that I would find a lot of descriptions of the differentials themselves; I did not expect, however, the amount of discussion about ideas that surround the differentials. Table 9 lists the totals of each type of theme for the entire data set and shows that throughout all of the interviews, there were eleven more occurrences of Tier 2 themes than the total occurrences of both types of Tier 1 themes. This suggests that the interview subjects had more to say about their ideas surrounding the differentials than about the characteristics of the differentials themselves. In other

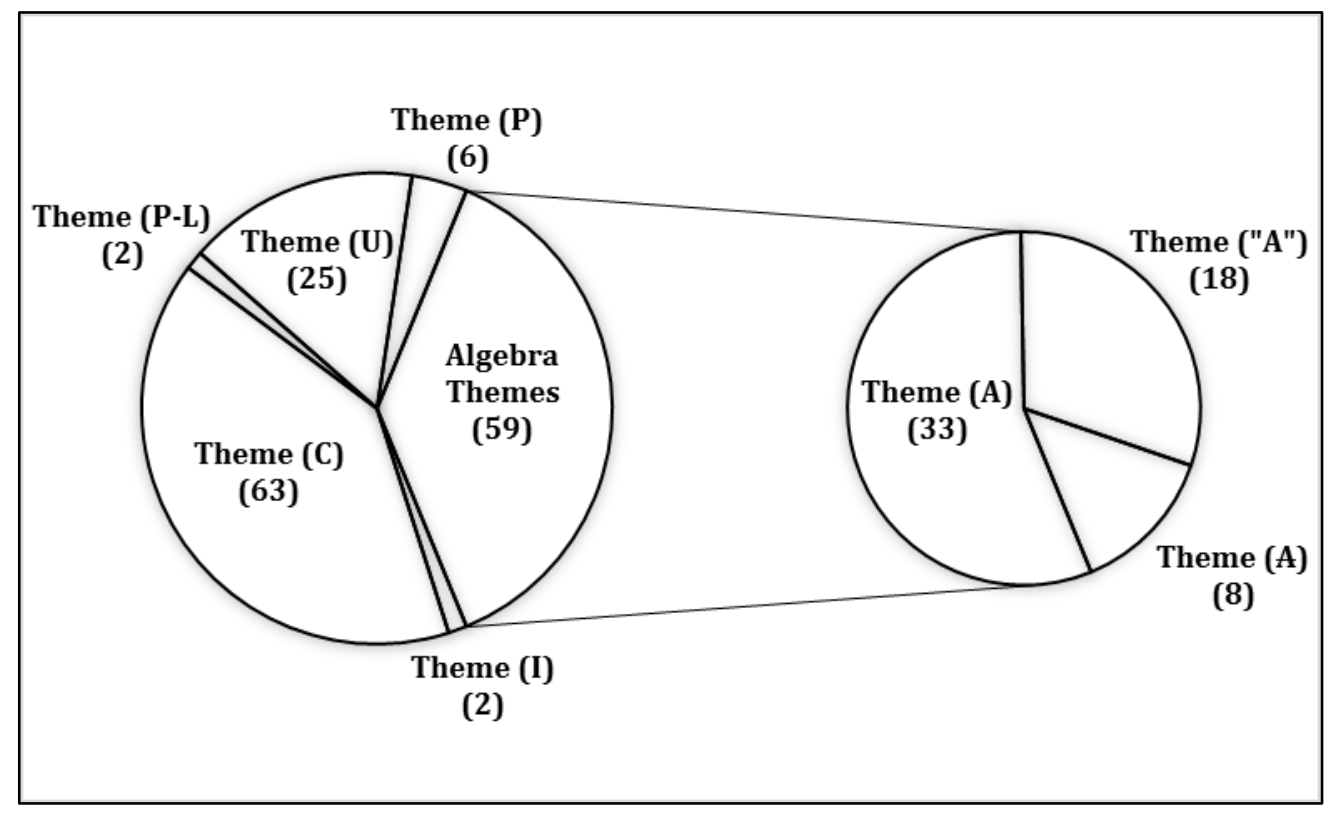

Figure 33: Total number of Tier 2 themes 


\begin{tabular}{|c|c|c|c|c|c|c|}
\hline \multicolumn{7}{|l|}{ Table 9} \\
\hline \multicolumn{7}{|c|}{ Total occurrences of all types of themes } \\
\hline $\begin{array}{l}\text { Tier 1: } \\
\text { Non-limit- } \\
\text { based }\end{array}$ & $\begin{array}{l}\text { Tier 1: } \\
\text { Limit- } \\
\text { based }\end{array}$ & $\begin{array}{c}\text { Tier 2: } \\
\text { (C)ontext }\end{array}$ & $\begin{array}{c}\text { Tier 2: } \\
\text { (U)ncertainty }\end{array}$ & $\begin{array}{c}\text { Tier 2: All } \\
\text { Algebra } \\
\text { Themes }\end{array}$ & $\begin{array}{c}\text { Other } \\
\text { Tier } 2 \\
\text { Themes }\end{array}$ & $\begin{array}{l}\text { No Tier: } \\
\text { "None" or } \\
\text { "Not Enough, } \\
\text { Information" }\end{array}$ \\
\hline$\overline{109}$ & 38 & 63 & 25 & 59 & 10 & 20 \\
\hline
\end{tabular}

words, within their concept images, ideas such as "context," "uncertainty," and "algebra" were just as important as their ideas of "the differential is an object/a pseudo-number/a real or infinitesimal number." Of course, it is possible that the interview questions skewed the results and inflated the importance of these ideas in these particular interview subjects. Nevertheless, the existence of these three "surrounding" ideas is not in question, and they can expand concept images beyond a list of simple statements that categorize differentials, giving individuals' concept images greater depth and nuance.

To conclude with a look at my specific results, anyone looking at this research will find that four of my interview subjects (André, Christopher, Eugene, and Gustav) used a total of six different Tier 1 themes during their interviews. This total could be considered extensive by itself, but it does not take into account any of the various Tier 2 themes of context, uncertainty, and algebra that might be attached to these Tier 1 themes, expanding and giving depth to the overall interpretation.

\section{What levels of consistency, if any, exist in the concept images of the differential within each individual?}

I would like to begin my answers of this and the next research question by showing some results from only one theme. Table 10 shows the responses of some interview subjects when discussing algebraic operations in Leibniz derivative notation, the separable ODE, and the 


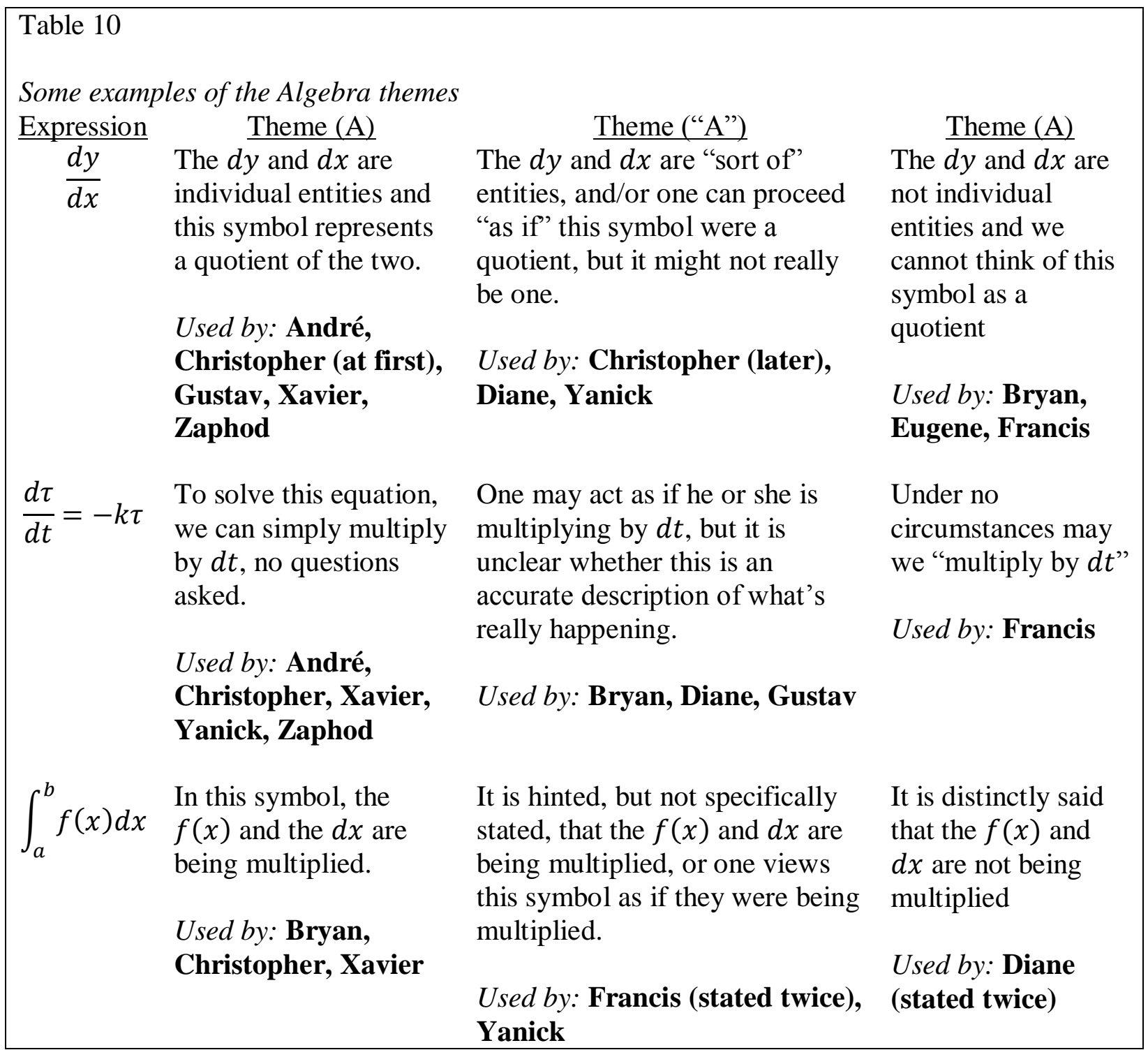

definite integral. When I initially described this theme, I noted that it might be possible that interview subjects could express multiple "algebra" views throughout his or her entire interview. An example of this can be found in the table: reading from bottom to top, Bryan believes that $f(x) d x$ is truly a multiplication, solving the ODE is only sort of, but not really, multiplying by $d t$, and the symbol $\frac{d y}{d x}$ is not a quotient. In these examples, the differential proceeds along a path that begins as "full-fledged participant in algebraic activities" and ends at "not even a mathematical object." 
This one example serves as a microcosm for the views of each interview subject. True, no interview subject's concept image was completely chaotic, bouncing wildly from conceptualization to conceptualization every time he or she saw a new expression. But at the other end, no interview subject's concept image was completely consistent throughout his or her interview. To give specifics, the number of Tier 1 themes utilized by my interview subjects ranged from two (Yanick and Zaphod) to six (André, Christopher, Eugene, and Gustav,) showing that not only did no interview subject stay consistent throughout his or her entire interview, but also that there were varying levels of this inconsistency.

Even the interview subjects who had the same level of consistency in their concept image by this measurement still invoked different ideas. Of the two interview subjects that only utilized two Tier 1 themes, Yanick viewed his differentials as either limit-based or non-limit-based pseudonumbers while Zaphod viewed his as formal hyperreal infinitesimals and variable indicators. The four interview subjects who utilized six Tier 1 themes did not all choose the same ones: Christopher used the most limit-based themes, André and Gustav viewed a lot of their differentials as welldefined values, and Eugene tended toward pseudo-numbers and variable indicators.

\section{What levels of consistency, if any, exist in the concept images of the differential among all mathematicians interviewed?}

There is less consistency in how all of the interview subjects view each expression's differentials. Tables in earlier sections of this dissertation have already shown the large collections of conceptualizations for Leibniz derivative notation and definite integrals that were used by my interview subjects. Table 10 shows additional inconsistency in how the group viewed the legality of algebraic operations with differentials. Indeed, each expression has interview subjects who 
champion the completely opposite views of Themes (A) and (A), and these completely opposite views are not necessarily championed by the same people from expression to expression.

To use the same metric used in the discussion of the previous question, the number of Tier 1 themes used by each interview expression ranged from two (the indefinite integral) to nine (definite and double integrals.) Since no expression was found to have only one view, I can only conclude that there is even less consistency among the views of all interview subjects than there is within the concept images of individual interview subjects. I can point to small islands of consistency, such as André and Bryan describing differentials akin to Courant and John's (1965) "physically infinitesimal" values, the three RUME faculty (Diane, Francis, and Yanick) having a greater reliance on limit-based themes than the other interview subjects, and Gustav and Zaphod making similar statements about Abraham Robinson's nonstandard analysis and hyperreal infinitesimal numbers.

I believe that I could expect to find instances of consistency if I interviewed a tailored group chosen for their specific interests. For example, a group of ten physicists might show consistency among their concept images, as might a group of ten differential geometers. However, using this research as a guide, I would now not expect either group to have complete agreement among their members, nor would I expect the two groups' views to be consistent with each other's. Regarding this dissertation, with its ten interview subjects with varied interests and backgrounds, it is clear that there is no meaningful consistency among all of their views.

\section{Summary of the Data and Results}

The amount of differing and sometimes contradictory responses found in each interview and among the discussions of each expression should put an end to the idea that there is one single, formal concept of the differential among all mathematicians. However, this lack of a formal 
concept definition does not mean that these interviews were fruitless, as they at least generated an initial list of categorizing themes. While the themes I have found can be used to describe each mention of differentials in my data, I would say that a fuller analysis of differential concept images requires more than just providing a list of themes. In the final section of this dissertation, I will attempt to discuss the teaching and learning implications that I believe are raised by some of these themes and based on some of the responses from my interview subjects. 


\section{DISCUSSION}

This dissertation not only presented a framework that can be used to conceptualize differentials, but also listed several themes that address ancillary considerations, such as the uncertainty that surrounds differentials or the validity of algebraic operations performed with differentials. Many of these differential conceptualizations and ancillary considerations have possible teaching implications that I shall discuss. Additionally in this chapter, I note limitations of this research and make recommendations for future studies

\section{Limitations of this Dissertation}

The first limitation I note is the absence of inter-rater reliability. During the long process of conducting the research and creating this dissertation, I had opportunities to present examples of my categories of differential conceptualizations to my advisor and other students authorized by my school's Institutional Review Board. While these other researchers gave me feedback and advice on the efficacy of these categories, no other researcher ever took any significant portion of interview data and attempted to categorize the differentials themselves. While some of the categories I have created are relatively easy to spot (examples include differentials as real or formal infinitesimals and differentials as variable indicators) some decisions (Does this differential truly result from a limit process? Which level of algebra is being performed with this differential? Is there enough "concreteness" to call this differential an object instead of an ephemeral idea?) were more difficult to make, and perhaps have the potential to be interpreted differently by other researchers.

Thus, it should not be assumed that all of my responses would be confirmed by other researchers, and I believe that my next research project should include co-researchers who have been briefed on my categorizations. Before undertaking any future research, I would present 
excerpts from existing transcripts and ask these co-researchers to independently categorize the differential views found within them. If enough discrepancies were found between my categorizations and theirs, I would lead a discussion to determine whether the discrepancies resulted from different understandings of my categorizations or perhaps even flaws in my framework. In either case, appropriate corrections, such as enriching my descriptions of themes or re-examining the accuracy of my framework, would be taken to ensure that all future research has inter-rater reliability.

Another limitation to this dissertation is the relatively small sample size. The framework and initial list of Tier 2 themes was generated by the data provided by seven interviews. On one hand, I am aware that a collection of seven interviews is generally not an extensive collection, and normally I would hesitate to make many sweeping generalizations from what might be viewed as a relatively small dataset. On the other hand, nothing in the second series of interviews contradicted the main themes in the flowchart I created, and I cannot foresee any future differential conceptualization that would not fit in my flowchart in some fashion.

While this leads me to believe that my flowchart is valid as it pertains to the main themes, the sheer amount of different Tier 1 subthemes and Tier 2 themes seems to suggest that I have but scratched the surface of the many subtleties that experts might have in their differential concept images. It would appear that future research with a greater number of subjects can explore not only how many additional Tier 2 themes and flowchart subthemes can be found but also how much nuance can be attached to each theme in my flowchart and whether more spaces might need to be added to accommodate a larger number of subthemes.

However, some subthemes in the flowchart might speak to an ignorance of nuance during my questioning. To give an example, in my data, Theme 3 is differential as pseudo-number and 
Theme 4 has subthemes of real number, formal infinitesimal, and 'one of those two but can't be determined." I now believe that last subtheme arose because my inexperience with the protocol blinded me to the fact that this subtheme is unnecessary with proper follow-up questions. If the interview subject describes a differential as a value but does not define it well, follow-up questions asking him or her to quantify it can be asked until the interview subject defines it as a real number, formal infinitesimal, or "not really a measurable value," which would make that differential a pseudo-number. The fact that "can't be determined" was an option in this dissertation shows that there were several instances in which I did not ask enough clarifying follow-up questions, which I shall endeavor to do in future research.

There is another reason that I might need to ask more clarifying, follow-up questions in future research. The first type of uncertainty discussed in my theme list was the existence and awareness of cognitive conflict. While two of my interview subjects, Francis and Yanick, noticed some conflict between some of their responses, Diane, Eugene, and Francis each mentioned a wish or hope that he or she was being consistent throughout all answers. These wishes perhaps point to the existence of an inherent drive for mathematicians to find clear, direct, and non-conflicting explanations for mathematical ideas. I submit that it is possible for this drive to shape interview subjects' responses toward greater consistency, especially when discussing a topic that has the potential to elicit many differing viewpoints, such as differentials. It is possible that I did not pay enough respect to this drive, and shall remember to do so in future research.

The last limitation I wish to note regards both the placement and wording of some of the expressions in my interview protocol. My interview protocol expressions labelled (6) was the "Law of Cooling" separable ODE $\frac{d \tau}{d t}=-k \tau$, and (7) was the definite integral $\int_{0}^{50} 700-3 x d x$ which represented the amount of work done in lifting a 400-pound weight halfway up the side of 
a building using a wire that weighed 3 pounds per foot. These were included to serve as

companions to the expressions (1) $\frac{d y}{d x}$ and (2) $\int_{a}^{b} f(x) d x$. I initially thought that as the interview subjects' proceeded from the earlier, context-free expressions to the later context-dependent expressions, their conceptualizations of the differentials involved might markedly change toward more "tangible," non-limit-based conceptualizations and away from limit-based and "not-astangible" conceptualizations.

However, this did not happen to the extent that I expected. While there was a slight version of this expected shift in the conceptualizations of the differentials from expressions (2) to (7), the opposite shift away from "tangible" differentials occurred between expressions (1) and (6). I theorize that a possible reason for this is the fact that the first thing I asked the interview subjects to do upon presenting the ODE was to solve it. Perhaps being asked to immediately solve the ODE caused the subjects to focus toward the elements of their concept images best suited for mathematical manipulations, such as differentials as automatic processes, and away from the elements of their concept images that endowed the differentials with a value. I believe that the idea of exploring how differential conceptualizations might vary if given a physical context is a good idea to explore in future research, but if I conduct this research, I will need to decide if there is a better and less-influencing way to introduce the ODE than immediately asking for a solution.

\section{Implications for Instruction}

The fact that expert mathematicians who are currently teaching at universities do not agree on a concept image for differentials can definitely have an effect on instruction. At the most basic level, it is not difficult to imagine a student taking both semesters of first-year calculus from professors who champion completely different views about differentials. If this hypothetical student is told in the first semester that differentials are limit-based pseudo-numbers that can be 
algebraically manipulated, and then told in the second semester that differentials are merely notation that may not be manipulated, then he or she is certainly not getting a consistent education, particularly if these calculus classes are followed by physics classes in which differentials are presented as non-limit-based physical entities.

If expert mathematicians can experience cognitive conflict with different elements of their concept image, then it stands to reason that students can experience it as well. My personal belief, which would theoretically eliminate much, if not all, such cognitive conflict, is that all differentials should be taught with the same conceptualization. Perhaps the idea of "one symbol; one meaning" for differentials is not appropriate and too strict to be used as an absolute in mathematics, but I would think that the current idea of "one symbol; a large collection of meanings, some of which are contradictory" is not any better.

My preference would be to teach differentials as non-limit-based informal hyperreal infinitesimals. Note that I do not propose introducing infinitesimals to students with a formal approach straight out of Robinson (1966), since such an approach would be beyond the understanding of the typical undergraduate student. ${ }^{12}$ Instead, I would advocate a more informal approach such as the approaches used by Keisler (2012), Henle and Kleinberg (2003), Hrbacek, Lessmann, and O’Donovan (2014), or Sloughter (2007), in which the arguments and evidence for the existence of infinitesimals are presented more informally. I suggest this approach because I believe that presenting differentials this way is the most logical and internally-consistent approach. It might eliminate, or at least lessen, the uncertainty that was common in my data, it avoids any

\footnotetext{
${ }^{12}$ I liken this to the way that many current textbooks address real numbers. They do not present students with a formal approach that mentions advanced ideas such as "Real numbers come from equivalence classes of convergent Cauchy series of rational numbers," but rather just assume that students have some informal and intuitive understanding of real numbers that does not need to be explored.
} 
confusion between limits and differentials, and it is beneficial for the transition between mathematics and other courses.

In my data, there were more instances of general uncertainty beyond obvious cognitive conflict. André and Eugene said that they did not have formal understandings or definitions for the term "differential" and the $d x$, respectively, while Bryan, Christopher, and Francis expressed that they were not comfortable with or did not like using phrases like "infinitely small" or "infinitesimally small" because such phrases were, as Bryan stated "hard to make it precise or practical in any way." It is certainly possible that such uncertainty can find its way into one's teaching. In her interview, Diane spoke of "hand-wavy" explanations given by books and remarked that, as an instructor, she is aware that some differential-based explanations are not developed well: "I feel like when we teach the $u$-substitution in particular, we just write this $[u=\sqrt{t}]$ and write that $\left[d u=\frac{1}{2 \sqrt{t}} d t\right]$, and we help them solve and plug stuff in, and we don't spend enough time talking about what is $d u$ and what is $d t$." If one were to adopt an infinitesimal-based calculus, then these particular instances of uncertainty could be addressed: all of the questions about the above definitions, phrases, and differentials can be answered by the central idea that differentials are infinitesimal values, with "infinitesimal" now formally defined.

Regarding limits and differentials: notations such as $\frac{d y}{d x}$ and $\int_{a}^{b} f(x) d x$ are used by both infinitesimal-based and limit-based calculus, even though the derivative and integral notations were created with Leibniz' infinitesimals in mind (Ely \& Boester, 2010; Keisler, 2012). These notations communicated the complex ideas of differentiation and integration via relationships between infinitesimals, even though, in Leibniz' time, these infinitesimals were not formallydefined. The development of the epsilon-delta mechanism did formalize these notations, but at the cost of removing them from their infinitesimal-based origins and placing them into a limit-based 
understanding. I believe that this removal and placement is a potential source of the multiple conceptualizations of differentials.

To use the definite integral notation $\int_{a}^{b} f(x) d x$ as an example, if one has adopted a nonstandard view of the notation, then the $d x$ will be a formally-defined infinitesimal amount. But if one adopts standard analysis, then their view of the differential will be shaped by how they view limits. To me, the idea of limits applied to infinitesimal-based notation is like a prism scattering one beam of light into many different colors: how does one choose to reconcile one's idea of "limit" with notational bits that initially represented infinitesimals? Does one try to relate the $d x$ directly to the Riemann sum's $\Delta x$ in any way? If so, is the $d x$ a transformed $\Delta x$ ? Is the $d x$ only a stage in the limiting process of taking $\Delta x$ to zero? Either way, does the $d x$ have a quantifiable size? If one chooses not to relate the $d x$ and $\Delta x$, does the $d x$ in the integral only serve as an indicator that a limit was taken? Does it serve to reference the variable of integration? Or is it possible that one views the $d x$ as having no meaning at all?

Each of these implications suggests to me that, as much as possible, limit-based teaching of differentials simply creates more problems than it solves, perhaps reinforcing my idea that teaching all differentials as informal hyperreal infinitesimals is preferable. Using infinitesimalbased calculus to teach concepts whose notation is built around infinitesimals should not be viewed as a radical idea, particularly when the alternative is using limits in notations not built for them.

Finally, my literature review contains many papers in which it is presumed that physicists generally view differentials as infinitesimal amounts (e.g. Artigue, 1991; Jones, 2013; López-Gay, Martínez Sáez, and Martínez Torregrosa, 2015). My Tier 2 Theme (C).b, the awareness of different interpretations depending upon the field of study, shows that some of my experts are aware that the physical sciences adopt a "differentials as infinitesimals" view. If one were to teach calculus 
with my suggested infinitesimal-based approach, then his or her students might find a transition to physics-based calculus easier.

However, I realize that this could be an option difficult to implement for some instructors. Not only is nonstandard analysis not widely accepted nor even well-known, but limits are ubiquitous throughout the current methods of calculus instruction, and I believe that it might be difficult for some to consider replacing limit-based calculus with infinitesimal-based calculus. If this is the case, I offer some possible justifications for trying such an approach and some suggestions for those who do not wish to do so.

First, within my Tier 1 themes, there were almost three times more non-limit themes than limit-based ones. I do acknowledge that it is possible that more-detailed questioning and/or further explorations of the interview subjects' concept images might have provided evidence that limits are considered more often than my research implies. However, I believe that the relatively small number of limit themes in the majority of differential conceptualizations is telling. If future research supports my data, then no matter how common limits are in calculus, the idea that differentials do not come from limits is at least common. Those who may not want to teach an infinitesimal-based course might at least consider teaching differentials as entities that exist without limit processes.

Another reason I suggest an infinitesimal-based calculus is because I feel that some statements and actions taken by some of my other interview subjects could be said to bear at least some resemblance to the presentation of nonstandard infinitesimals. Christopher described his $d y$ and $d x$ in Leibniz derivative notation by a process of zooming into a curve using a graphing utility until the section of curve in the viewing window appeared linear. His $d y$ and $d x$ were described as the vertical and horizontal components of this seemingly-linear bit. The result of this process 
mirrors Keisler's (2012) use of an "infinitesimal microscope" to focus on a section of a curve to such a degree that it appears linear, and assign $d y$ and $d x$ to the vertical and horizontal components of this linear bit. André and Diane described certain differentials as "the $d x$ is what's left of $\Delta x$ after it goes to zero" and "then this point starts to kind of approach that point [tracing this in Figure D3], and when that point is exactly on top of this point, then the $\Delta y$, we're going to think about as $d y, \Delta x$ we're going to think about as $d x$," respectively. Both of these descriptions seem to include the contradictory ideas that the measurable amount of the quantity in question is zero but there is still some presence of that quantity remaining. Keisler's (2012) and other nonstandard analysis works could be said to address that seeming contradiction by use of infinitesimals and the standard part function. A formal infinitesimal, $\epsilon$ still has a presence in the hyperreal numbers, yet the standard, or real, part of $\epsilon$ is zero.

I do not claim that Christopher, André, and Diane are actually using nonstandard analysis in their explanations, but I see similarities in their responses to nonstandard analysis. Thus, I would suggest that calculus instructors could easily consider a nonstandard, infinitesimal-based approach to calculus, because they already hold informal views that align very well to nonstandard views.

Finally, even if one does not wish to adopt an infinitesimal-based calculus, at the very least I believe that steps should be taken to avoid or at least minimize uncertainty and cognitive conflict when teaching differentials. Of course, conflict could be avoided by teaching only one conceptualization for differentials, but if an instructor wants to use different conceptualizations at different times, he or she should include caution when introducing the new uses for differentials, making sure that all explanations for any new uses do not contradict previous explanations. An example of this is how many of my interview subjects defined the "Work" problem: the integral is built using a differential that represents a small amount of wire, and then the integral is evaluated 
using the Fundamental Theorem of Calculus, at which time the differential is used to indicate the variable of integration. Explanations like this, in which the reasons for different conceptualizations are specifically indicated, should serve to minimize any potential cognitive conflict among students who come into contact with multiple conceptualizations. I would also suggest that some classroom discussion of the historical development of differential notation might be similarly beneficial, as multiple differential conceptualizations might be better accepted by students who are aware that the differential-containing Leibniz notations can be defined and accepted both with and without the use of limits.

\section{Possible Future Research}

Regardless of which of these specific teaching implications I will choose to investigate further, the first step in such an investigation must be to conduct this research with a much larger sample size. It has been suggested to me that some type of internet-based survey might be beneficial, as I would probably be able to attract a greater number of busy, time-strapped interview subjects by offering of a fifteen-minute internet survey as opposed to a forty-five minute more formal interview. An internet-based study would also give me the opportunity to at least partially randomize the order of the expressions I would present, allowing me to ensure that interview subjects' responses are not necessarily influenced by the order in which the questions were presented.

Regarding the teaching implications that interest me the most, I should like to focus initially on the divide between mathematics differentials and physics differentials and the efficacy of nonstandard-analysis-based calculus instruction. I think that both of these ideas could be combined by proposing, exploring, and/or experimenting with a first-year calculus course with an infinitesimal-based curriculum. In this hypothetical curriculum, differentials would be viewed as 
separate, individual entities that exist without limits; this would be a definition more in-line with both the "physics" conceptualization and the intended meaning of those notations.

Other possible studies can be postulated from the teaching implications I have listed. A study could be conducted that explores the differences in the views of the legality of performing algebraic operations with differentials. Another study could attempt to measure how deeply the ideas of limit processes affects one's view of differentials, and whether those ideas are helpful or harmful. In all of these potential studies, attention could be focused on the uncertainty of the interview subjects' responses, or, alternatively, a study could be conducted with the sole purpose of gauging the level of uncertainty about differentials that is admitted by the interview subjects.

A final idea I would like to explore in future research is how individuals interpret the symbolic forms of the expressions containing differentials. Since differentials rarely exist in a vacuum but usually exist as a part of a larger expression, it could be argued that one's conceptualization of a particular differential might have some relation to one's conceptualization of the expression in which the differential resides. When exploring the question of what it truly meant for students to understand physics equations, Sherin (2001) described what he called the symbolic forms of equations. These symbolic forms contain both the physical layout of the symbols used in a particular equation (the symbol template) and the meaning behind the particular form chosen for the equation (the conceptual schema.) I argue that while these symbolic forms were initially created as a method to delve into student understanding of the meaning of physics equations, I believe that a similar symbolic awareness of how one views differential-containing expressions might be helpful in exploring differential conceptualizations.

I already mentioned Jones' (2013) study in which, after students discussed and used the definite integral in a variety of situations, the student responses were analyzed so as to try to 
determine the conceptual schemas behind their answers. He identified three symbolic forms of the definite integral expression, each using the symbol template $\int_{[]}^{[]}[] d[]$, but containing different conceptual schema. First, in the "adding up pieces" symbolic form, where one divides the region indicated by the definite integral into rectangles and adds the resulting areas of all such rectangles, the $d x$ represents the width of each rectangle. Second, in the "perimeter and area" symbolic form, where the region indicated by the definite integral is simply viewed as a geometric area, the $d x$ represents that the $x$-axis is a border of the region. Third, in the "function matching" symbolic form, where the integrand is viewed as the derivative of an unknown function, the $d x$ indicates that the integrand had been differentiated with respect to $x$.

In this research, I identified two instances in which interview subjects described symbolic forms that were not found in any other responses. First, Diane was the only interview subject who, at times, discussed the differential as two separate and meaningful letters, discussing the " $d$ " of the differential as follows:

$\ldots$ if I want to refer to that quantity and I think of this $\Delta y$ as being an infinitely small change with respect to $y$, then I would write it with a $d$ instead of a $\Delta$, and I actually say that that's because the $\Delta$ is a capital " $\mathrm{D}$ ", and then the $d$ is kind of like the lowercase, so that's why we switch over to that when we're talking about the infinitely small quantity.

When the idea in the above quote is combined with the idea that the second letter of the differential indicates the variable of integration, I would suggest that she assigned to certain differentials the symbolic form [][] instead of [], the initial $d$ suggesting that the quantity being discussed needs to be understood as an "infinitely small" quantity, and the terminal letter suggesting a particular variable. Her "pseudo-number" differentials, which were not related to a 
particular direction, would be assigned the symbolic form [], as they would be thought of as one unit. This is not to say that no other interview subjects entertains similar variation in his symbolic forms, but other than Christopher mentioning that a " $d$ " represented the existence of a limit, no other interview subject addressed the " $d$ " in the differential separately.

Second, Zaphod's idea that the left-hand side of the ODE represents an operator $\frac{d}{d t}$ applied to $\tau$ instead of the combination $\frac{d \tau}{d t}$ suggests a different symbolic form. Based on the previous interviews, I would have expected one of two symbolic forms for the left-hand side of the ODE $\frac{d \tau}{d t}=f(\tau) g(t)$. If one believes that one can multiply by $d t$, I would expect the symbolic form $\frac{[]}{[]}$, which shows the independence of the $d \tau$ and the $d t$. If, however, one does not believe that multiplying by $d t$ is acceptable, I would expect the form [ ], which would show the idea that $\frac{d \tau}{d t}$ is not a quotient. However, Zaphod seems to be using a different symbolic form, $\frac{d}{d[]}$, in which the only replaceable consonant on the left-hand side is the one that determines the variable of integration.

I can foresee instances in which different interpretations of differentials can lead to the same expression being considered with other symbolic forms. To give some example, two possible symbolic forms of Leibniz derivative notation are $\frac{[]}{[]}$ or simply [ ], where the first is meant to indicate that the expression consists of a $d y$ separated from a $d x$, while the second is meant to indicate that the entire symbol should not be read as two separate entities but instead as one notation. Similarly, one's symbolic form for the separable ODE $\frac{d y}{d x}=f(x) g(y)$ could influence the choice of solution method. If one views the equation with the symbolic form $\frac{[]}{[]}=[][]$, then one could think it acceptable to "multiply both sides by $d x$ ", since the $d x$ seems to be a separate 
entity. However, if one uses the form []$=[][]$, then such multiplication might not be allowed in his or her conceptual schema. Finally, the symbol for indefinite integration could either be interpreted with the symbolic form $\int[][]$, in which the $d x$ would seem to be considered equally

valid as the integrand, or the more basic form $\int[]$, in which the integrand and $d x$ are merged, and thus the $d x$ would have no individual meaning.

Diane's and Zaphod's examples were noted here because those were the only instances in my data in which symbolic forms were used that were noticeably different from the other interview subjects' symbolic forms. This does not mean, however, that the more-common symbolic forms and how these forms relate to their individual's concept image are not worthy of analysis. Such analysis was beyond the scope of this dissertation, and is also a candidate for future work.

\section{Final Thought}

Regardless of what, if any, future research I might choose to explore, I would submit that this dissertation has provided me with a renewed appreciation for differentials. Christopher closed his interview by saying:

[Differentials are] very useful, 'cause they have a lot of content. There's a lot of, sort of conceptual content in there, and if you shy away from them, you're robbing the students of sort of conceptual content where they can think about things. These things actually mean something, rather than being things that are so abstruse that they can only be handled with a course in advanced calculus. I think a lot of that all that developed just from physical reasoning and - although the mathematics by itself is not rigorous, you can make it rigorous, and the reasoning is valid. So I don't see any reason to avoid talking about them 
I would agree with Christopher that differentials are meaningful and can be presented as being more than obscure entities only understood with advanced mathematics. As I proceed with my teaching career, I shall aim to use them when teaching lower-level calculus, while being aware of the many possible interpretations of them that were discovered during this research. 


\section{REFERENCES}

Artigue, M. (1991). Chapter 11 - Differentiation. In D. Tall (Ed.), Advanced mathematical thinking. Dordrecht; Boston: Kluwer Academic Publishers.

Artigue, M., Menigaux, J., \& Viennot, L. (1990). Some aspects of students' conceptions and difficulties about differentials. European Journal of Physics, 11(5), 262.

Artigue, M., \& Viennot, L. (1987). Some aspects of students' conceptions and difficulties about differentials. In J.D. Novak (ed.) Misconceptions and educational strategies in science and mathematics: Proceedings of the Second International Seminar Misconceptions and Educational Strategies in Science and Mathematics, July 26-29 1987, Cornell University, Ithaca, NY, USA. Cornell University.

Barnett, R. A. \& Ziegle, M. R. (1989). Applied mathematics for business, economics, life sciences, and social sciences ( $3^{\text {rd }}$ ed.). San Francisco, CA: Dellen publishing Company

Boyce, W. E. \& DiPrima, R. C. (2012). Elementary Differential Equations and Boundary Value Problems $\left(10^{\text {th }}\right.$ ed.). Hoboken, NJ: John Wiley and Sons, Inc.

Braun, V., \& Clarke, V. (2006). Using thematic analysis in psychology. Qualitative research in psychology, 3(2), 77-101.

Breusch, R., \& Ogilvy, C.S. (1969). Calculus and analytic geometry. Boston, MA: Prindle, Weber, \& Schmidt, Incorporated.

Courant, R., \& John, F. (1965). Introduction to Calculus and Analysis (Vol. I). New York, NY.: Interscience Publishers.

Dray, T. (2013). Using Differentials to Differentiate Trigonometric and Exponential Functions. College Mathematics Journal, 44(1), 17-23.

Dray, T., \& Manogue, C. A. (2010). Putting Differentials Back into Calculus. College Mathematics Journal, 41(2), 90-100.

Dunn, J. W., \& Barbanel, J. (2000). One model for an integrated math/physics course focusing on electricity and magnetism and related calculus topics. American Journal of Physics, 68(8), 749-757.

Ellis, R. \& Gulick, D. (1988). Calculus with analytic geometry (Alternate Ed.) San Diego, CA: Harcourt, Brace, Jovanivich.

Ely, R. (2010). Nonstandard Student Conceptions about Infinitesimals. Journal for Research in Mathematics Education, 41(2), 117-146.

Ely, R., \& Boester , T. (2010). Point/Counterpoint: Should we teach calculus using 
infinitesimals? Paper presented at the Thirteenth SIGMAA on Research in Undergraduate Mathematics Education Conference.

http://sigmaa.maa.org/rume/crume2010/Abstracts2010.htm

Fisher, R. C. \& Ziebur, A. D. (1965). Calculus and analytic geometry (2 ${ }^{\text {nd }}$. ed.). Englewood Cliffs, NJ: Prentice-Hall, Inc.

Freudenthal, M. (1973). Mathematics as an educational task. Dordrecht-Holland: D. Reidel Publishing Company.

Gleason, A. M., Hughes-Hallett, D., \& McCallum, W.G. (2013). Calculus: Single Variable (6th ed.) New York: Wiley.

Henle, J. M., \& Kleinberg, E. M. (2003). Infinitesimal calculus: Courier Corporation.

Henry, V. (2010). An Introduction to Differentials Based on Hyperreal Numbers and Infinite Microscopes. PRIMUS, 20(1), 39-49.

Hrbacek, K., Lessmann, O., \& O'Donovan, R. (2014). Analysis with Ultrasmall numbers: Chapman and Hall/CRC.

Hsu, E. (2013). Spreadsheet of North American Doctoral Programs in Math Education [Excel Spreadsheet]. Retrieved from http://sigmaa.maa.org/rume/phd.html.

Hu, D., \& Rebello, N. S. (2013). Understanding Student Use of Differentials in Physics Integration Problems. Physical Review Special Topics - Physics Education Research, 9(2), 020108-020101-020108-020114.

Hughes-Hallet, D., McCallum, W. G., Gleason, A., Osgood, B. G., Lock, P. F., Quinney, D., Flath, D. E., Rhea, K., Lomen, D. O., Tecosky-Feldman, J., Lovelock, D., \& Tucker, T. W. (2006). Applied calculus ( ${ }^{\text {rd }}$ ed.). Hoboken, NJ: John Wiley \& Sons, Inc.

Jones, S. R. (2013). Understanding the integral: Students' symbolic forms. The Journal of Mathematical Behavior, 32(2), 122-141. Retrieved from http://www.sciencedirect.com/science/article/pii/S0732312312000612. doi:http://dx.doi.org/10.1016/j.jmathb.2012.12.004

Jones, S. R. (2015). Areas, anti-derivatives, and adding up pieces: Definite integrals in pure mathematics and applied science contexts. The Journal of Mathematical Behavior, 38, 928. Retrieved from http://fulltext.study/download/360655.pdf.

Keisler, H. J. (2012). Elementary calculus: An infinitesimal approach: Courier Corporation.

Larson, R., \& Edwards, B. H. (2014). Calculus of a single variable: Early transcendental functions: Nelson Education. 
López-Gay, R., Martínez Sáez, J., \& Martínez Torregrosa, J. (2015). Obstacles to Mathematization in Physics: The Case of the Differential. Science and Education, 24(56), 591-613.

Martínez-Torregrosa, J., Gras-Martí, A., \& López-Gay, R. (2006). Mathematics in physics education: scanning historical evolution of the differential to find a more appropriate model for teaching differential calculus in physics. Science et education., 15.

McCarty, T., \& Sealey, V. (2017). Experts' Varied Concept Images of the Symbol dx in Integrals and Differential Equations. Paper presented at the 20th Annual Conference on Research in Undergraduate Mathematics Education, San Diego, CA.

McCarty, T., \& Sealey, V. (2018, February). How Experts Conceptualize Differentials: The Results of Two Studies. Poster session presented at the $21^{\text {st }}$ Annual Conference on Research on Undergraduate Mathematics Education, San Diego, CA.

Mizrahi, A. \& Sullivan, M. (1982). Calculus and analytic geometry. Belmont, CA: Wadworth Publishing Company

Nguyen, D.-H., \& Rebello, N. S. (2011). Students' difficulties with integration in electricity. Physical Review Special Topics-Physics Education Research, 7(1), 010113. Retrieved from http://link.aps.org/pdf/10.1103/PhysRevSTPER.7.010113.

Oehrtman, M. (2009). Collapsing Dimensions, Physical Limitation, and Other Student Metaphors for Limit Concepts. Journal for Research in Mathematics Education, 40(4), 396-426. Retrieved from http://www.jstor.org/stable/40539345.

Orton, A. (1983). Students' understanding of differentiation. Educational studies in mathematics, 14(3), 235-250.

Rees, P. K. \& Sparks, F.W. (1969). Calculus with analytic geometry. New York, NY: McGrawHill.

Robinson, A. (1966). Non-standard analysis. Amsterdam: North-Holland Publishing Co.

Roundy, D., Dray, T., Manogue, C. A., Wagner, J. F., \& Weber, E. (2015, March). An extended theoretical framework for the concept of derivative. In Proceedings of the 18th Annual Conference on Research in Undergraduate Mathematics Education (pp. 838-843).

Sealey, V. (2008) Calculus Students' Assimilation of the Riemann Integral into a Previously Established Limit Structure (Doctoral dissertation). Arizona State University, Tempe, AZ

Sealey, V. (2014). A framework for characterizing student understanding of Riemann sums and definite integrals. The Journal of Mathematical Behavior, 33, 230-245. doi:http://dx.doi.org/10.1016/j.jmathb.2013.12.002 
Sealey, V., \& Thompson, J. (2016). Student interpretation and justification of "backward" definite integrals. Paper presented at the 19th Annual Conference on Research in undergraduate Mathematics Education, Pittsburgh, PA.

Sherin, B. L. (2001). How students understand physics equations. Cognition and instruction, 19(4), 479-541.

Sloughter, D. (2009). Yet another calculus text: A short introduction with infinitesimals. Retreived from http://yact.synechism.org/yact-05102007.pdf

Stein, S. K. (1967). Calculus in the first three dimensions. New York, NY: McGraw-Hill Book Company

Stewart, J. (1987). Calculus. Belmont, CA: Wadsworth, Inc.

Stewart, J. (2015). Calculus: Early Transcendentals Cengage Learning.

Strauss, A., \& Corbin, J. M. (1997). Grounded theory in practice. Sage.

Tall, D. (1993). Students' difficulties in calculus. In proceedings of working group (Vol. 3, pp. 13-28).

Tall, D., \& Vinner, S. (1981). Concept Image and Concept Definition in Mathematics with Particular Reference to Limits and Continuity. Educational Studies in Mathematics, 12(2), 151-169.

Thompson, S. P. (1910). Calculus made easy: being a very-simplest introduction to those beautiful methods of reckoning which are generally called by the terrifying names of the differential calculus and the integral calculus. London; New York: Macmillan; St. Martin's P.

Thurston, H. (1972). What Exactly Is dy/dx? educstudmath Educational Studies in Mathematics, 4(3), 358-367.

Vinsonhaler, R. (2016). Teaching Calculus with Infinitesimals. Journal of Humanistic Mathematics, 6(1), 249-276.

Von Korff, J., \& Rebello, N. S. (2012). Teaching integration with layers and representations: A case study. Physical Review Special Topics-Physics Education Research, 8(1), 010125. Retrieved from http://link.aps.org/pdf/10.1103/PhysRevSTPER.8.010125.

Zandieh, M. (2000). A theoretical framework for analyzing student understanding of the concept of derivative. CBMS Issues in Mathematics Education, 8, 103-127.

Zill, D. G. (1997). A first course in differential equations with modeling applications $\left(6^{\text {th }}\right.$ ed.). Pacific Grove, CA: Brooks/Cole Publishing Company. 


\section{APPENDIX A: Interview Protocol for the Exploratory Study}

Introduction (to be read to the subject): I am interviewing experts to ask them about their perspectives in various calculus topics. I am more concerned with how you think about the topics than how you would teach the topics, although I do understand that how you teach a topic and how you view a topic might be related. Please answer the following questions with as much detail as you can.

1. Consider the following symbols (each of which contains the symbol $d x$ ):
(1) $\quad \int f(x) d x$
(2) $\quad \int_{a}^{b} f(x) d x$
(3) $\quad \int_{b}^{a} f(x) d x$
a. What is the role of $d x$ in each expression? (What does it mean?)
b. Is there a graphical meaning for $d x$ ?
c. How does $\int_{a}^{b} f(x) d x$ compare to $\int_{b}^{a} f(x) d x$ ? How do you know?
d. Can you justify the last answer in terms of area under a curve?

2. Consider the following symbols, (each of which contains the symbol $d x$ ):

$$
\begin{aligned}
& \text { (4) If } y=y(x) \text {, the symbol } \frac{d y}{d x} \\
& \text { (5) If } y=y(x), d y=y^{\prime}(x) d x \\
& \text { (6) If } x=x(t) \text {, the symbol } \frac{d x}{d t} \\
& \text { (7) If } x=x(t), d x=x^{\prime}(t) d t
\end{aligned}
$$

a. What is the role of $d x$ in each expression? (What does it mean?)

3. Consider two things:

(8) The integral: $\int_{1}^{4} \frac{\cos \sqrt{x}}{2 \sqrt{x}} d x$

(9) The beginning steps in the integral's evaluation:

$$
\begin{gathered}
\text { Given } \int_{1}^{4} \frac{\cos \sqrt{t}}{2 \sqrt{t}} d t \\
\text { Let } x=\sqrt{t} \\
d x=\frac{1}{2 \sqrt{t}} d t \\
t=1 \text { and } 4 \text { mean that } x=1 \text { and } 2 \text {, respectively } \\
\text { The integral becomes } \int_{1}^{2} \cos x d x
\end{gathered}
$$

a. What are the roles of the $d x$ symbols in both (8) and (9)? 
4. Consider the following two ODEs:

- Separable ODEs can be described as equations that are of the form (10), and a common solution method is to begin by putting the equation into form (11) and then integrating, as shown here:

$$
\begin{gathered}
\text { (10) } \quad \frac{d y}{d x}=g(y) h(x) \\
p(y) d y=h(x) d x \text {, where } p(y)=\frac{1}{g(y)} \\
\text { (12) } \int p(y) d y=\int h(x) d x
\end{gathered}
$$

- The equation (13) $\left(2 x y-9 x^{2}\right) d x+\left(x^{2}+2 y+1\right) d y=0$

is an Exact ODE, verified by showing that $\frac{\partial}{\partial y}\left(2 x y-9 x^{2}\right)=\frac{\partial}{\partial x}\left(x^{2}+2 y+1\right)=2 x$ with an implicit solution of (14) $x^{2} y-3 x^{3}+y^{2}+y=C$.

a. The manipulation from (10) to (11) might be construed as "multiplying both sides by $d x$." Is "multiplying by $d x$ " what is really happening here? If so, then do you claim that algebraic manipulation with this $d x$ is possible? If not, then what is really happening here?

b. What is the role of $d x$ in the separable ODE? Does this role change from (10) through (12)?

c. What is the role of $d y$ in the separable ODE? Does this role change from (10) through (12)?

d. What are the roles of $d x$ and $d y$ in the exact ODE?

e. Can $d x$ and $d y$ have a numerical role? If so, can they be negative?

f. Is it mathematically legal to rewrite the original ODE into the form $\frac{d y}{d x}=\frac{9 x^{2}-2 x y}{x^{2}+2 y+1}$ ? If so, what are the roles of $d y$ and $d x$ now? 


\section{APPENDIX B: Interview Protocol for the Pilot Study}

\section{Introduction}

To be read at the beginning of the interview: I am interviewing experts to attempt to gain an understanding of how they view various calculus topics. I would like to get as full of an understanding as possible, so please answer the following questions with as much detail as you can.

Once we're settled in: Tell me about your credentials. What are your degrees? Major and minor areas of study? Teaching experience?

\section{$\underline{\text { Part 1A - Integrals }}$}

$$
\text { (1) } \quad \int_{a}^{b} f(x) d x \quad \text { and } \quad(2) \quad \int f(x) d x
$$

- What do these expressions mean to you?

- Can you tell me what you believe is the role of each piece in these expressions? Ask

- If they still haven't really described the $d x$ : Can you tell me (again) what you believe is the role the $d x$ in these expressions?

- Can there be a graphical meaning to these expressions? (Then just the $d x$, if needed)

- If needed: [Ask about the "size" of the $d x$ ]

- Comment on the similarities/differences between (2) and (1)

(3) An object is moving along a straight line at a nonconstant velocity (denoted by $v$ ) over a four-minute period.

- What is the meaning of the integral $\int_{0}^{4} v d t$ ?

- Can you tell me again what you believe is the role of each piece in this expression?

- Specifically, what is the role of the $d t$ in this expression?

- ["Graphical" and/or "Size" questions, as appropriate]

- Do you think about the units to help understand the problem? What would the units of $v$ and $d t$ be? Which entity "has" the units: the $d t$ or the $t$ ?

\section{$\underline{\text { Part 1B - Derivatives }}$}

$$
\text { (4) } \frac{d y}{d x}
$$

- What does this expression mean to you?

- If that first question wasn't answered clearly: Some people view the symbol as a ratio between two terms: " $d y$ " and " $d x$ " - others view the symbol as one notation, in which the " $d y$ " and " $d x$ " are not individual terms. What do you believe?

- Can you tell me (again) what you believe are the roles (if any) of the $d y$ and $d x$ in these expressions?

- ["Graphical" and/or "Size" questions, as appropriate] 
- Can you go back and remind me - the earlier differentials were Do you think about the $d y$ and $d x$ in these cases the same or different?

(5) Please solve the ODE $\frac{d u}{d t}=\frac{2 t}{\cos u}$

- [Basically, what manipulation are they doing to get it in the form $\int \cos u d u=\int 2 t d t$ ? Specifically, does $d t$ have a "quality" that allows it to be multiplied?]

- What are the roles of $d t$ and $d u$ in this ODE?

- Are they as before? [Compare with the $d x$ and $d y$ in (4)]

- Do the roles change at all during the solution? [Compare with the $d x$ in (2)]

- Can you go back and remind me - the earlier differentials were . Do you think about the $d t$ and $d u$ in these cases the same or different?

\section{$\underline{\text { Part 1C - Differential of a Function }}$}

$$
d y=y^{\prime}(x) d x
$$

- What does this expression mean to you?

- Can you tell me what you believe is the role of each piece in these expressions?

- Can you tell me (again) what you believe are the roles of the $d y$ and $d x$ in this expression?

- ["Graphical" and/or "Size" questions, as appropriate]

- To link this with differentiation (4): Is (6) equivalent to $\frac{d y}{d x}=y^{\prime}(x)$ ? Specifically, does $d x$ have a "quality" that allows it to be divided?

- Can you go back and remind me - the earlier differentials were Do you think about the $d y$ and $d x$ in these cases the same or different?

$$
\text { [Ask the subject to evaluate (7) } \left.\int_{1}^{4} \frac{\cos \sqrt{t}}{2 \sqrt{t}} d t\right]
$$

- How do you conceptualize the $d t$ and $d u$ in (7)?

- Is there any change in the $d u$ between $d u=\frac{1}{2 \sqrt{t}} d t$ and $\int_{1}^{2} \cos u d u$ ? What about in the quality of the differentials $\int_{1}^{4} \frac{\cos \sqrt{t}}{2 \sqrt{t}} d t$ and $\int_{1}^{2} \cos u d u$ ? Compare with (1) and (6)

- Do any answers change if asked to evaluate $\int \frac{\cos \sqrt{t}}{2 \sqrt{t}} d t$ (indefinite integral) instead?

- Can you go back and remind me - the earlier differentials were . Do you think about the $d t$ and $d u$ in these cases the same or different?

\section{$\underline{\text { Part 1D - Deltas and Infinitesimals }}$}

- To you, what is the difference (if any) between a $\Delta x$ and a $d x$ ?

- Does this difference between $d x$ and $\Delta x$ extend to all versions of $d x$ and $\Delta x$ ? 
- Show the subject $(8)^{13}$ and say Lopez-Gay, et al., accepted two different ways to begin the solution - Can you comment on the validity of each solution method? Be clear on what they like and what they don't. Then: Is there a particular solution method that conforms to your views of the characterization of the $\Delta V$ and $d V$ ?

- A lot of times you hear the phrases "infinitely small" or "infinitesimal amount". Do you use phrases like those? When?

- What do you mean by "[whatever phrase they used]"?

- How many "infinitely small"s does it take to go from 0 to 1? Depending on their answer: Are we outside the Real Numbers? Are you OK with that?

- Comment on the following idea: "It is OK to conceptualize differentials such as the dx in $\int_{a}^{b} f(x) d x$ or $\frac{d y}{d x}$ as nonzero, finite quantities, small enough to fall under any measurable scale." (For reference: Diameter of electron: less than $10^{-16} \mathrm{~cm} /$ Smallest unit of time ever measured: $10^{-21} \mathrm{sec}$ )

\section{$\underline{\text { Part } 2 \text { - All Versions and Preferences for Students }}$}

For each of these perspectives, would you be OK with your students viewing the $d x$ this way? Which of these perspectives do you think are useful for students and which of them do you think are limiting/incorrect?

$$
\text { (1) } \quad \int_{a}^{b} f(x) d x
$$

a) The entire integral symbol is some sort of specialized antidifferentiation cue - the $d x$ is merely notation that denotes the variable of integration.

b) The entire integral symbol is the limit of a Riemann Sum and the $d x$ is some sort of referent to the $\Delta x$ - the $d x$ is the "limit" of the $\Delta x$ in a Riemann Sum or the $\Delta x$ that has been passed through the limit.

c) The entire integral symbol is the (infinite) sum of an infinite number of products between the real, finite $f(x)$ and an infinitesimal $d x$ - the $d x$ is an infinitesimal amount

$$
\text { (2) } \quad \int f(x) d x
$$

a) The entire integral symbol is some sort of general antidifferentiation cue - the $d x$ is merely notation that denotes the variable of integration

b) The entire integral symbol some sort of limit of an "infinite" Riemann Sum and the $d x$ is some sort of referent to the $\Delta x$ - the $d x$ is the "limit" of the $\Delta x$ in a Riemann Sum or the $\Delta x$ that has been passed through the limit.

c) The entire integral symbol is the (infinite) sum of the displacement given by an infinite amount of products between the real, finite $f(x)$ and an infinitesimal $d x$ - the $d x$ is an infinitesimal amount of horizontal displacement

d) The entire integral symbol is or some version of a definite integral of the form $\int_{x_{0}}^{x} f(t) d t$, and thus the $d x$ is however the subject perceived the $d x$ in the definite integral.

${ }^{13}$ (8) is the problem from López-Gay, Martínez Sáez, \& Martínez Torregrosa (2015), p 611. 
(4) $\frac{d y}{d x}$

a) The symbol is only a notation for "the derivative of $y$ with respect to $x$," - the $d y$ and $d x$ are not two separate entities.

b) The symbol represents the limit of a difference quotient, specifically $\lim _{\Delta x \rightarrow 0} \frac{\Delta y}{\Delta x}-$ the $d y$ and $d x$ are referents to the $\Delta y$ and $\Delta x$ or the $\Delta y$ and $\Delta x$ that have been passed through the limit.

c) The symbol represents the ratio (or the standard part of the ratio) between an infinitesimal $d y$ and an infinitesimal $d x$ - both are infinitesimal amounts.

$$
d y=y^{\prime}(x) d x
$$

a) This is only a notation we use when we need it - the individual differentials have no meaning.

b) This notation represents the idea that, in a small neighborhood around $x$, the tangent line is an approximation of the curve $y(x)-$ the $d x$ is the same as a $\Delta x$, and the $d y$ is an approximation to $\Delta y$.

c) This notation represents that $d y$ is an infinitesimal that is proportional to an infinitesimal $d x-$ both $d y$ and $d x$ are well-defined infinitesimals. 


\title{
APPENDIX C: Consent Form for the Dissertation Study
}

\section{W- WestVirginiaUniversity.}

\section{Research Informed Consent Only Minimal Risk}

\author{
Differentials Study \\ Principal Investigator \\ Dr. Vicki Sealey \\ Department \\ Mathematics \\ Protocol Number \\ 1710807177 \\ Study Title \\ Co-Investigator \\ Sponsor (if any) \\ How Mathematicians and Physicists Conceptualize Differentials \\ Tim McCarty \\ N/A
}

\section{Contact Persons}

If you have any questions, concerns, or complaints about this research, you should contact Dr. Vicki Sealey at (304) $293-5329$. For information regarding your rights as a research subject, to discuss problems, concerns, or suggestions related to the research, to obtain information or offer input about the research, contact the Office of Research Integrity \& Compliance at (304) $293-7073$.

\section{Introduction}

You, have been asked to participate in this research study, which has been explained to you by . This study is being conducted by Tim McCarty, under the supervision of Dr. Vicki Sealey, in the

Department of Mathematics at West Virginia University.

\section{Purpose(s) of the Study}

How experts conceptualize differentials in various contexts is being analyzed in this study.

\section{Description of Procedures}

This study involves video recording of interviews with participants, where we will discuss differentials as found in varied contexts. Interviews are expected to last approximately 45 minutes. You will be asked to think out loud and share the ways in which you think about the topics. You are welcome to use pen and paper, if you wish. Excerpts of written work may be published, and it is possible that your handwriting could be recognized.

\section{Alternatives}

You do not have to participate in this study.

\section{Benefits}

You may not receive any direct benefit from this study. The knowledge gained from this study may eventually benefit others in the teaching and learning of concepts involving differentials.

\section{Discomforts}

There are no known or expected risks from participating in this study.

\section{Financial Considerations}

There are no fees or payment for participating in this study.

\section{Confidentiality}

Any information about you that is obtained as a result of your participation in this research will be kept as confidential as legally possible. Video recordings will be kept locked up and/or stored on a password protected, secure service. Data will be kept for a minimum of 3 years and will be destroyed as soon as the research is finished. 


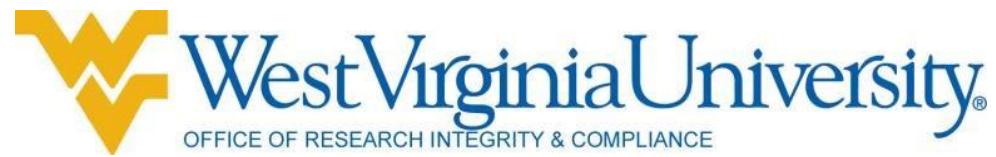

\section{Research Informed Consent Only Minimal Risk}

In any publications that result from this research, your name will not be published, but it is possible that your handwriting could be recognized.

\section{Voluntary Participation}

Participation in this study is voluntary. You are free to withdraw your consent to participate in this study at any time. Refusal to participate or withdrawal will involve no penalty to you. In the event new information becomes available that may affect your willingness to participate in this study, this information will be given to you so that you can make an informed decision about whether or not to continue your participation.

You have been given the opportunity to ask questions about the research, and you have received answers concerning areas you did not understand. By signing below, you acknowledge that you willingly consent to participate in this research.

\section{Signatures}

Signature of Subject

Printed Name

Date

Time

The participant has had the opportunity to have questions addressed. The participant willingly agrees to be in the study.

Signature of Investigator or Co-Investigator

Printed Name

Date

Time 


\section{APPENDIX D: Interview Protocol for the Dissertation Study}

\section{$\underline{\text { Introduction }}$}

1. Read the following: I am interviewing experts to attempt to gain an understanding of how they view various calculus topics. I would like to get as full of an understanding as possible, so please answer the following questions with as much detail as you can.

2. Tell me about your credentials. Specifically, what are your degrees? What were your major and minor areas of study? What is your teaching experience?

3. What does the word "differential" mean to you?

\section{Expressions without Context: Sheet \#1 (Derivative Notation)}

Show the subject a sheet of paper with the following expression on it:

$$
\frac{d y}{d x}
$$

1. Can you give me a 30" answer of what this expression means to you? Then: If you could give me a more in-depth answer to this question: How do you conceptualize the $d y$ and $d x$ in this expression?

2. Do you think about a graphical representation for the $d y$ and $d x$ in (1)?

3. How would you characterize/define the size, if any, of the $d y$ and $d x$ in (1)?

\section{Expressions without Context: Sheet \#2 (One-Dimensional Integrals)}

Show the subject a sheet of paper with the following expressions on it:

$$
\int_{a}^{b} f(x) d x
$$

1. Can you give me a 30"' answer of what these expressions mean to you? Then: If you could give me a more in-depth answer to this question: How do you conceptualize the $d x$ in each expression?

2. Do you think about a graphical representation for the $d x$ in (2)? What about the $d x$ in (3)?

3. How would you characterize/define the size, if any, of the $d x$ in (2)? In (3)? 


\section{Expressions without Context: Sheet \#3 (Two-Dimensional Integral)}

Show the subject a sheet of paper with the following expression on it:

$$
\int_{0}^{1} \int_{2}^{3} f(x, y) d y d x
$$

1. Can you give me a 30" answer of what this expression means to you? Then: If you could give me a more in-depth answer to this question: How do you conceptualize the $d y$ and $d x$ in this expression?

2. Do you think about a graphical representation for the $d y$ and $d x$ in (4)?

3. How would you characterize/define the size, if any, of the $d y$ and $d x$ in (4)?

\section{Expressions without Context: Sheet \#4 (Relationship Between Differentials)}

Show the subject a sheet of paper with the following expression on it:

$$
d y=2 x d x
$$

1. Can you give me a 30" answer of what this expression means to you? Then: If you could give me a more in-depth answer to this question: How do you conceptualize the $d y$ and $d x$ in this expression?

2. Do you think about a graphical representation for the $d y$ and $d x$ in (5)?

3. How would you characterize/define the size, if any, of the $d y$ and $d x$ in (5)? 
$\underline{\text { Expressions within a Context: Sheet \#1 (Derivative Notation) }}$

Show the subject a sheet of paper with the following on it:

To model the temperature of a 20-degree cup of water that is placed in a zero-degree environment, we can use the following:

$$
\frac{d \tau}{d t}=-k \tau, \quad \tau(0)=20
$$

1. This is a separable differential equation. Could you please show me how you would solve it and explain how you conceptualize the differentials in each step?

2. How do you conceptualize the $d \tau$ and $d t$ in (6)?

Expressions within a Context: Sheet \#2 (Integral Notation)

Show the subject a sheet of paper with the following on it:

A cable weighing three pounds per foot is dangled from the roof of a 100-foot tall building and attached to a 400-pound weight resting on the ground. The amount of work required to lift the weight halfway up the building can be found by

$$
\text { (7) } \int_{0}^{50} 700-3 x d x
$$

1. Read the text to them and say: How do you conceptualize the " $d x$ " in (7)? 


\section{Expressions within a Context: Sheet \#3 (Relationships Between Differentials)}

Show the subject a sheet of paper with the following on it:

\begin{tabular}{|ccc|}
\hline Evaluate the integral & $\int_{1}^{4} \frac{\cos \sqrt{t}}{2 \sqrt{t}} d t$ & \\
Solution: & $I=\int_{1}^{4} \frac{\cos \sqrt{t}}{2 \sqrt{t}} d t$ & \\
& Let & \\
& $I=\int_{1}^{2} \cos u d u=\sqrt{t}$ \\
$I=\sin 2-\sin 1$ & & \\
\end{tabular}

1. Given the integral $\int_{1}^{4} \frac{\cos \sqrt{t}}{2 \sqrt{t}} d t$, the following steps show a possible evaluation method. Do you agree with this evaluation method? Is this the method you would use to evaluate this integral? If not, would you show me the method you would use to evaluate this integral?

2. Write an “(8)" next to the step $d u=\frac{1}{2 \sqrt{t}} d t$ and ask: How do you conceptualize the $d u$ and $d t$ in (8)?

3. Do you think about a graphical representation for the $d u$ and $d t$ in (8)?

4. How would you characterize/define the size, if any, of the $d u$ and $d t$ in (8)? 


\section{$\underline{\text { Deltas and Infinitesimals }}$}

NOTE: There is no particular time when the following questions will be asked. The first set will be asked the first time the subject mentions the idea of "Delta" and the second set will be asked the first time the subject mentions a descriptive phrase like "a small amount," "infinitely small," or "infinitesimally small." If, for some reason, the subject never mentions Deltas of descriptive phrases like those, these questions will be asked after the previous sheet is completed.

After they have mentioned a $\Delta x$

1. To you, what is the difference (if any) between a $\Delta x$ and a $d x$ ?

2. Do you think about a graphical representation for the $\Delta x$ ?

3. How would you characterize/define the size, if any, of the $\Delta x$ ?

After a descriptive, "small" phrase is used

1. What do you mean by "[whatever phrase they used]"?

2. If no such "infinitely/infinitesimally" phrase has been used, I will ask: A lot of times you hear the phrases "infinitely small" or "infinitesimal amount". Do you use phrases like those? When?

3. Do you think about a graphical representation for [their phrase]?

4. How would you characterize/define the size, if any, of [their phrase]? 